Measurements of channels and time delay of light

in strongly scattering media

Jeroen Bosch 


\section{Measurements of channels and time delay of light in strongly scattering media}

Metingen aan kanalen en de propagatietijd van licht in sterk verstrooiende media 


\section{Cover}

Transmitted field of a scattering invariant mode

Bosch, Jeroen

Measurements of channels and time delay of light

in strongly scattering media

J. Bosch - Utrecht

Universiteit Utrecht

Faculteit Bètawetenschappen

Departement Natuur- en Sterrenkunde

Thesis Universiteit Utrecht - With a summary in Dutch

ISBN: 978-94-640-2345-9 


\section{Measurements of channels and time delay of light in strongly scattering media}

Metingen aan kanalen en de propagatietijd van licht in sterk verstrooiende media

(met een samenvatting in het Nederlands)

\section{Proefschrift}

ter verkrijging van de graad van doctor aan de

Universiteit Utrecht

op gezag van de

rector magnificus, prof. dr. H.R.B.M. Kummeling, ingevolge het besluit van het college voor promoties

in het openbaar te verdedigen

op donderdag 2 juli 2020

des middags te 4.15 uur

door

\section{Jeroen Bosch}

geboren op 14 september 1992

te Davos, Zwitserland 
$\begin{array}{ll}\text { Promotor: } & \text { Prof. dr. A. P. Mosk } \\ \text { Copromotor: } & \text { Dr. ir. S. Faez }\end{array}$

This work is financially supported by the Nederlandse Organisatie voor Wetenschappelijk Onderzoek (NWO). 


\section{Contents}

1. Introduction 1

2. Apparatus for full field control 11

2.1. Introduction . . . . . . . . . . . . . . 11

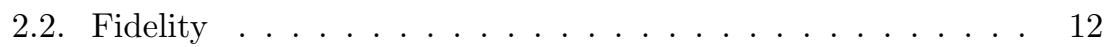

2.2.1. Fidelity of a transmission matrix . . . . . . . . 12

2.2.2. Fidelity of digital optical phase conjugation . . . . . 13

2.3. Hardware . . . . . . . . . . . . . . . . . . . . . . 14

2.3.1. Laser module . . . . . . . . . . . . . . . . . . . . 14

2.3.2. Interferometry . . . . . . . . . . . . . . . . 14

2.3.3. Field synthesis . . . . . . . . . . . . . . . 17

2.3.4. Sample holder . . . . . . . . . . . . . . . . 19

2.4. Sample. . . . . . . . . . . . . . . . . . . . 21

2.4.1. Sample thickness . . . . . . . . . . . . . . 22

2.5. Physical alignment . . . . . . . . . . . . . . . 22

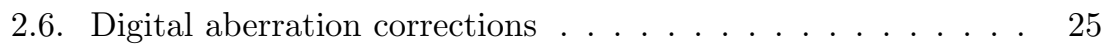

2.6.1. Dispersion correction . . . . . . . . . . . . . . . 29

2.7. Transmission matrix measurements . . . . . . . . . . . . 29

2.7.1. Matrix normalization . . . . . . . . . . . 30

2.8. Phase noise in transmission matrix measurement . . . . . . . 31

2.9. Wavelength dependent phase drift correction . . . . . . . 32

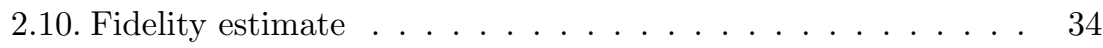

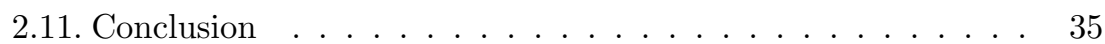

3. Coupling light to transmission channels 39

3.1. Introduction . . . . . . . . . . . . . . . . . 39

3.2. Transmission matrix measurement . . . . . . . . . . . . 40

3.3. Coupling light to transmission channels . . . . . . . . . 42 
3.4. Calculating transmittance from an independent transmission matrix . . . . . . . . . . . . . . . 45

3.5. Conclusions . . . . . . . . . . . . . . . 47

4. Spectral width of transmission channels 53

4.1. Introduction . . . . . . . . . . . . . . . 53

4.2. Theory ...................... 54

4.3. Correlation function from a multi spectral transmission matrix measurement . . . . . . . . . . . . . . . . 55

4.4. Wavelength dependence of transmission enhancement of chan-

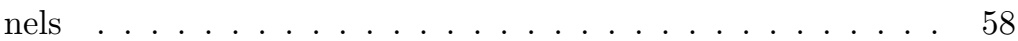

4.5. Anomalous transmittance of transmission channels . . . . . . 62

4.6. Influence of fidelity on transmission enhancement . . . . . . . 64

4.7. Correlation width of transmission channels . . . . . . . . . . . 69

4.8. Conclusions . . . . . . . . . . . . . . . . 72

5. Time delay of transmission channels 77

5.1. Introduction . . . . . . . . . . . . . . . 77

5.2. Coherent multispectral transmission matrix . . . . . . . . 78

5.3. Transmission time delay of transmission channels . . . . . . . 79

5.4. Conclusions . . . . . . . . . . . . . . . . 85

6. Measurements of time delay eigenstates of light transmitted $\begin{array}{lr}\text { through scattering media } & 89\end{array}$

6.1. Introduction . . . . . . . . . . . . . . . . . 89

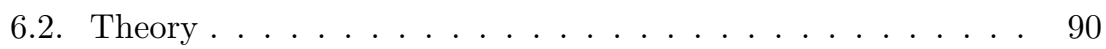

6.3. Measurement of time delay eigenstates . . . . . . . . . . 91

6.4. Consistency checks . . . . . . . . . . . . . . . . . . 95

6.5. Properties of time delay eigenstates . . . . . . . . . . . . 97

6.5.1. Correlation width of time delay eigenstates . . . . . . 99

6.6. Conclusions . . . . . . . . . . . . . . . . . . . 101

$\begin{array}{ll}\text { 7. Scattering invariant modes } & 105\end{array}$

7.1. Introduction . . . . . . . . . . . . . . 105

7.2. Finding scattering invariant modes . . . . . . . . . . . 106

7.3. Superpositions of scattering invariant modes . . . . . . . . . . 110

7.4. Sparse scattering invariant modes . . . . . . . . . . . . 112 
7.5. Conclusion ..................... 114

8. Iterative algorithm for finding scattering invariant modes $\quad 117$

8.1. Introduction . . . . . . . . . . . . . . . . 117

8.2. Methods . . . . . . . . . . . . . . 117

8.3. Simulation of iterative algorithm for finding a SIM . . . . . . 119

8.4. Finding SIM through digital optical phase conjugation . . . . 121

8.5. Conclusion .................. . 123

A. Appendix 125

A.1. Speckle correlation function . . . . . . . . . . . . 125

A.2. Zernike polynomials . . . . . . . . . . . 126

$\begin{array}{lr}\text { Summary and outlook } & 129\end{array}$

$\begin{array}{lr}\text { Samenvatting } & 133\end{array}$

$\begin{array}{lr}\text { Dankwoord } & 137\end{array}$

$\begin{array}{lr}\text { Over de auteur } & 139\end{array}$ 



\section{Introduction}

We observe most of the world around us with our eyes. Light is scattered and reflected from objects into our eyes which allows us to see the objects. Another instance of light scattering can be found in clouds and fog. In clouds and fog there are many small droplets of water that scatter light in different directions. Light reflected off an object is scattered in the fog and loses its information about the object such that an object deep in the fog cannot be seen. The difference between a dense layer of fog, in which nothing can be seen, and a thin layer of fog is the average number of light scattering events between the observer and the object. In a thin fog, only some of the light is scattered, and the object is still visible. However, in a thick fog most or all of the light is scattered multiple times making it impossible to see through. In many everyday objects, such as milk, human tissue, paint, paper or cloth a similar scattering process occurs, where light is scattered by the structure of the material. In milk for example the light is scattered from the fat and protein globules suspended in the liquid.

Light transport through diffusely scattering media can be described through mesoscopic transport theory [1-4]. We are interested in the diffusive regime, where the following inequality holds [2]

$$
\lambda<l \ll L \ll L_{\mathrm{abs}},
$$

where $\lambda$ is the wavelength of the light inside the material, $l$ the mean free path which is the average distance light travels between scattering events, $L$ the size of the object and $L_{\text {abs }}$ the absorption length. In this regime the diffusion approximation describes the average intensity of light transported through a diffusive sample to a very good approximation [2]. However, many physical phenomenon in diffusive media can only be described by including light interference effects, for example enhanced backscattering [5-8].

The full knowledge of the transmitted field of a specific sample is mathematically represented by its transmission operator (TO), which maps any 
incident field to the corresponding transmitted field. In slab-type samples it is typically impossible to completely know the transmission operator experimentally as it represents all incident and transmitted waves, and a portion of these will always fall outside the apertures of any apparatus. The TO can be approximated by measuring a matrix of complex amplitude transmission coefficient between a finite set of incident and transmitted modes, known as the transmission matrix (TM). The first transmission matrix measurement by Popoff and colleagues [9] sampled only a small fraction of the transmission operator, such that the transmission eigenvalue statistics resembled that of a random matrix [10], later measurements show a signature of the underlying mesoscopic statistics [11-15]. Controlled coupling of light to specific modes of strongly scattering media was demonstrated first by focusing scattered light to a single point through wavefront shaping of the light incident on the scattering medium [16]. A key question is how well a measured TM represents the properties of the TO. In this thesis, we propose a convenient figure of merit, the fidelity, that addresses this question. We design an apparatus that can probe multispectral transmission properties with unprecedentedly high fidelity.

A key concept in mesoscopic transport theory is that of transmission channels. These channels form an orthogonal set of incident fields that remains orthogonal upon propagation through the sample. They correspond to the singular value decomposition of the transmission matrix. The distribution of their singular values in strongly scattering media was theoretically analyzed by Dorohkov, Mello, Perreira and Kumar [17,18]. The surprising result was that open channels, which are fully transmitted, occur in any diffusive sample, as long as absorption can be ignored. A first demonstration of coupling light to these open channels was provided through wavefront shaping [19]. Calculations using random matrix theory [20] have shown that a high experimental fidelity is needed to observe them. The spectral bandwidth of the transmission channels is of importance for their practical application [21,22]. It can also give an insight to its relation to resonances [23]. Measurements have been performed in microwave waveguides with a limited number of modes $[24,25]$. Optical measurements in a diffusive system were performed on a single transmission channel with limited transmittance [26]. Numerical work was done in the localized [27] and the diffusive regime [28] for a 2D waveguide geometry. 
Light pulses that are transmitted though a diffusely scattering medium are spread out in time. The transmitted pulses are stretched and transmitted with a complicated speckle-like time-dependence. There are ensemble averaged results for the time delay of light in a multiple scattering medium [29], however the time delay of individual modes such as the transmission channels or time delay eigenstates remains unexplored.

The time delay eigenstates, which are solutions of the Wigner-Smith time delay operator of the sample $[30,31]$ are fields that are scattered with a welldefined time delay. Experiments demonstrate focusing of light at specific time delays through wavefront shaping [32-34]. The measurement of multispectral and time resolved transmission matrices [35-41], has opened up the possibility of measuring the time delay of transmission channels and finding the time delay eigenstates. So far, the measurements of multispectral transmission matrices in scattering media that have been reported only recover a small fraction of the information in the transmission operator. These measurements therefore cannot be used to probe the time delay eigenstates or the time delay properties of transmission channels. In this thesis we recover a large fraction of the information in the transmission operator and report measurements of the time delay eigenstates of light in a scattering medium and the time delay properties of transmission channels.

Another set of special fields are scattering invariant modes (SIMs). These are fields that are transmitted the same through a scattering medium and a layer of air, except for a phase and amplitude difference. They represent the field that is transmitted through the scattering sample as if not interacting. Therefore they may hold relevance for imaging modalities that rely on this non-interacting light, such as optical coherence microscopy. This may be especially relevant in relatively sparsely scattering samples. We demonstrate different strategies for finding SIMs and we report on the properties of the SIMs that we found.

\section{Outline of this thesis}

In this thesis we present measurements of key properties of the transport of light through random scattering media that have not been studied before in experiment. We elucidate the frequency-domain correlation width and 
time delay of transmission channels and show the first measurements, to our knowledge, of time delay eigenstates in strongly scattering samples. We also show the scattering invariant modes and introduce a method for physically solving complex valued eigenvalue problems which we use to find a scattering invariant mode through iterative phase conjugation.

In Chapter 2 we describe the experimental apparatus and measurement procedures that we use for our different measurements. We define the goals and properties of the apparatus, introduce the multispectral transmission matrix measurement procedure and derive a measure for the fidelity of the transmission matrix.

In Chapter 3 we demonstrate the experimental capabilities of our measurements by measuring the transmission matrix of a multiple scattering material and calculating the transmission channels. We define methods to measure the transmittance. We measure the transmittance both by projecting the transmission channels on the multiple scattering medium and through the transmission matrices.

In Chapter 4 we show measurements of the spectral line shape of transmission channels. We determine different regimes of the transmittance, and compare the results for different sample thicknesses. We also investigate the influence of the fidelity of the measured transmission matrix on the results.

In Chapter 5 we investigate the time delay of the transmission channels. We introduce a method to measure the phase delay time of a transmission channel. We calculate the time delay of the transmission channels with different transmittance and perform several consistency checks to rule out artifacts of the measurement.

In Chapter 6 we show the first results of the time delay eigenvalues of a multiple scattering material. We measure the time delay eigenvalues for different sample thicknesses and show their distribution. Finally we measure the transmittance of the time delay eigenvalues and how they decorrelate when changing the wavelength of the incident light.

In Chapter 7 we report measurements of scattering invariant modes, which are waves that are transmitted the same through two different materials. We present experimental results and physically propagate the fields through a strongly scattering sample and air. We show that we can find and generate fields that highly correlate between these two media. Finally we introduce sparse scattering invariant modes, which allow us to transmit 
simple images through two distinct media.

In Chapter 8 we show a method to find a single scattering invariant mode by a digital optical phase conjugation procedure. We investigate the convergence of the measurement procedure which requires a symmetry breaking term to converge, and show experimental results.

Finally, in Chapter 9 we summarize the results and give an outlook for future research. 


\section{Bibliography}

[1] C. W. J. Beenakker, "Random-matrix theory of quantum transport," Rev. Mod. Phys. 69, 731-808 (1997).

[2] M. C. W. van Rossum and T. M. Nieuwenhuizen, "Multiple scattering of classical waves : microscopy , mesoscopy, and diffusion," Rev. Mod. Phys. 71, 313-371 (1999).

[3] E. Akkermans and G. Montambaux, Mesoscopic Physics of Electrons and Photons (Cambridge University Press, 2007).

[4] S. Rotter and S. Gigan, "Light fields in complex media: Mesoscopic scattering meets wave control," Rev. Mod. Phys. 89, 015005 (2017).

[5] Y. N. Barabanenkov, "On wave corrections for the transfer equation for backward scattering." Izvestiia Vysshaia Uchebn. Zaved., Radiofizika 16, 88-96 (1973).

[6] Y. Kuga and A. Ishimaru, "Retroreflectance from a dense distribution of spherical particles," J. Opt. Soc. Am. A 1, 831-835 (1984).

[7] M. P. V. Albada and A. Lagendijk, "Observation of weak localization of light in a random medium," Phys. Rev. Lett. 55, 2692-2695 (1985).

[8] P.-E. Wolf and G. Maret, "Weak localization and coherent backscattering of photons in disordered media," Phys. Rev. Lett. 55, 2696-2699 (1985).

[9] S. Popoff, G. Lerosey, R. Carminati, M. Fink, A. Boccara, and S. Gigan, "Measuring the transmission matrix in optics: An approach to the study and control of light propagation in disordered media," Phys. Rev. Lett. 104, 100601 (2010).

[10] V. A. Marčenko and L. A. Pastur, "Distribution of eigenvalues for some sets of random matrices," Math. USSR Sb. 1, 457-483 (1967).

[11] M. Kim, Y. Choi, C. Yoon, W. Choi, J. Kim, Q.-H. Park, and W. Choi, "Maximal energy transport through disordered media with the implementation of transmission eigenchannels," Nat. Photonics 6, 581-585 (2012).

[12] D. Kim, W. Choi, M. Kim, J. Moon, K. Seo, S. Ju, and W. Choi, "Implementing transmission eigenchannels of disordered media by a binarycontrol digital micromirror device," Opt. Commun. 330, 35 - 39 (2014).

[13] S. M. Popoff, A. Goetschy, S. Liew, A. Stone, and H. Cao, "Coherent control of total transmission of light through disordered media," Phys. 
Rev. Lett. 112, 133903 (2014).

[14] W. Choi, M. Kim, D. Kim, C. Yoon, C. Fang-Yen, Q.-H. Park, and W. Choi, "Preferential coupling of an incident wave to reflection eigenchannels of disordered media," Sci. Rep. 5, 11393 (2015).

[15] M. Kim, W. Choi, C. Yoon, G. H. Kim, S. hyun Kim, G.-R. Yi, Q.H. Park, and W. Choi, "Exploring anti-reflection modes in disordered media," Opt. Express 23, 12740-12749 (2015).

[16] I. M. Vellekoop and A. P. Mosk, "Focusing coherent light through opaque strongly scattering media," Opt. Lett. 32, 2309-2311 (2007).

[17] O. N. Dorokhov, "On the coexistence of localized and extended electronic states in the metallic phase," Solid State Commun. 51, 381-384 (1984).

[18] P. A. Mello, P. Pereyra, and N. Kumar, "Macroscopic approach to multichannel disordered conductors," Ann. of Phys. 181, 290-317 (1988).

[19] I. M. Vellekoop and A. P. Mosk, "Universal optimal transmission of light through disordered materials," Phys. Rev. Lett. 101, 120601 (2008).

[20] A. Goetschy and A. D. Stone, "Filtering random matrices: The effect of incomplete channel control in multiple scattering," Phys. Rev. Lett. 111, 063901 (2013).

[21] A. P. Mosk, A. Lagendijk, G. Lerosey, and M. Fink, "Controlling waves in space and time for imaging and focusing in complex media," Nat. Photonics 6, 283 (2012).

[22] D. A. B. Miller, "Waves, modes, communications, and optics: a tutorial," Adv. Opt. Photon. 11, 679-825 (2019).

[23] J. Wang and A. Z. Genack, "Transport through modes in random media," Nature 471, 345-348 (2011).

[24] A. Peña, A. Girschik, F. Libisch, S. Rotter, and A. Chabanov, "The single-channel regime of transport through random media," Nat. Commun. 5, 3488 (2014).

[25] M. Davy, Z. Shi, J. Wang, X. Cheng, and A. Z. Genack, "Transmission eigenchannels and the densities of states of random media," Phys. Rev. Lett. 114, 033901 (2015).

[26] J. Bosch, S. A. Goorden, and A. P. Mosk, "Frequency width of open channels in multiple scattering media," Opt. Express 24, 26472-26478 (2016).

[27] Z. Shi and A. Z. Genack, "Dynamic and spectral properties of transmis- 
sion eigenchannels in random media," Phys. Rev. B 92, 184202 (2015).

[28] C. W. Hsu, A. Goetschy, Y. Bromberg, A. D. Stone, and H. Cao, "Broadband coherent enhancement of transmission and absorption in disordered media," Phys. Rev. Lett. 115, 223901 (2015).

[29] I. M. Vellekoop, P. Lodahl, and A. Lagendijk, "Determination of the diffusion constant using phase-sensitive measurements," Phys. Rev. E 71, 056604 (2005).

[30] E. P. Wigner, "Lower limit for the energy derivative of the scattering phase shift," Phys. Rev. 98, 145-147 (1955).

[31] F. T. Smith, "Lifetime matrix in collision theory," Phys. Rev. 118, 349-356 (1960).

[32] J. Aulbach, B. Gjonaj, P. M. Johnson, A. P. Mosk, and A. Lagendijk, "Control of light transmission through opaque scattering media in space and time," Phys. Rev. Lett. 106, 103901:1 -4 (2011).

[33] O. Katz, E. Small, Y. Bromberg, and Y. Silberberg, "Focusing and compression of ultrashort pulses through scattering media," Nat. Photonics 5, 372-377 (2011).

[34] D. J. McCabe, A. Tajalli, D. R. Austin, P. Bondareff, I. A. Walmsley, S. Gigan, and B. Chatel, "Spatio-temporal focusing of an ultrafast pulse through a multiply scattering medium," Nat. Commun. 2, 447 (2011).

[35] Y. Choi, T. R. Hillman, W. Choi, N. Lue, R. R. Dasari, P. T. C. So, W. Choi, and Z. Yaqoob, "Measurement of the time-resolved reflection matrix for enhancing light energy delivery into a scattering medium," Phys. Rev. Lett. 111, 243901 (2013).

[36] S. Kang, S. Jeong, W. Choi, H. Ko, T. D. Yang, J. H. Joo, J.-S. Lee, Y.-S. Lim, Q.-H. Park, and W. Choi, "Imaging deep within a scattering medium using collective accumulation of single-scattered waves," Nat. Photonics 9, 253 (2015).

[37] D. Andreoli, G. Volpe, S. Popoff, O. Katz, S. Grésillon, and S. Gigan, "Deterministic control of broadband light through a multiply scattering medium via the multispectral transmission matrix," Sci. Rep. 5, 10347 (2015).

[38] M. Mounaix, D. Andreoli, H. Defienne, G. Volpe, O. Katz, S. Grésillon, and S. Gigan, "Spatiotemporal coherent control of light through a multiple scattering medium with the multispectral transmission matrix," Phys. Rev. Lett. 116, 253901 (2016). 
[39] R. French, S. Gigan, and O. L. Muskens, "Speckle-based hyperspectral imaging combining multiple scattering and compressive sensing in nanowire mats," Opt. Lett. 42, 1820-1823 (2017).

[40] J. Carpenter, B. J. Eggleton, and J. Schröder, "Complete spatiotemporal characterization and optical transfer matrix inversion of a 420 mode fiber," Opt. Lett. 41, 5580-5583 (2016).

[41] M. Mounaix, H. Defienne, and S. Gigan, "Deterministic light focusing in space and time through multiple scattering media with a time-resolved transmission matrix approach," Phys. Rev. A 94, 041802 (2016). 



\section{Apparatus for full field control}

\subsection{Introduction}

In this chapter, we describe the experimental apparatus, the procedures, algorithms and samples used in our experiments. The goal of our measurements is to study the properties of transmission channels of strongly scattering samples, and to observe wavelength dependent behavior. To observe the transmission channels, we accurately sample the transmission operator as multispectral transmission matrices [1-3] and calculate the sample properties from the transmission matrices. The open transmission channels [4-6], which are due to the mesoscopic correlations, can only be meaninfully studied if we accurately sample a large fraction of the transmission matrix [7,8]. Additionally, we want to be able to perform digital optical phase conjugations, which allows us to find so called scattering invariant modes as described in Chapter 8.

The apparatus has multiple functionalities. Firstly it measures transmission matrices at a high fidelity. Second it physically propagates arbitrary vectors of the transmission matrix through the sample. Finally we can perform a phase, amplitude and polarization controlled digital optical phase conjugation. All these functionalities can be performed as a function of wavelength, which also allows us to measure multispectral transmission matrices.

This chapter is structured as follows. We first define a figure of merit for our measurements, which is the fidelity $F$ of the transmission matrix. Then we introduce the apparatus and its different modules with their functionality and our samples. Next we describe the physical and software alignment procedure that is utilized for the phase conjugation and the transmission matrix measurements. We introduce the measurement procedure for the transmission matrices, where we quantify the phase noise and correct for 
phase drift in our transmission matrix measurements. Finally we make an estimate of the fidelity of our transmission matrix measurement.

\subsection{Fidelity}

In the context of wavefront shaping and in some discussions of the Loschmidt paradox, the fidelity of the reconstruction of a field is defined as

$$
F=|\gamma|^{2}=\left|\left\langle E_{\text {target }} \mid E_{\text {approx }}\right\rangle\right|^{2},
$$

where the field $E_{\text {target }}$ represents the field that an ideal method aims to synthesize or predict, and $E_{\text {approx }}$ is the result of an experimental or numerical approximation procedure (such as wavefront shaping or the prediction of a transmitted field using a transmission matrix) [9, 10]. Both fields are assumed to be normalized. If the target and actual fields are identical up to a global prefactor the fidelity $F=1$, whereas if the experimental procedure results in a random field the fidelity is small and of the order of the inverse number of degrees of freedom.

In most optical experiments the fidelity is constrained by two limitations: The fact that only a fraction of the incident and outgoing modes can be detected due to apertures in real and angular space, and noise on the amplitude measurements. The effect of uncorrelated measurement noise is known to be mostly to reduce fidelity by a factor $\cos ^{2} \delta \phi$, where $\delta \phi$ represents the phase noise in the system [11]. Other effects such as phase drift, polarization cross talk, the imaging resolution and overlap between the base vectors further reduce the fidelity but are minimized by the choice of a suitable basis vectors [3]. In the remainder we will ignore the effect of noise and study the fidelity of transmission matrix measurements as a result of aperture effects.

\subsubsection{Fidelity of a transmission matrix}

For clarity we restrict ourselves to a transmission matrix in a waveguide geometry, so that the transmission matrix is square and of well-defined dimension $N \times N$. We assume the true transmission matrix is $T_{\text {true }} \equiv T_{\mathrm{t}}$ and its approximation $T_{\text {approx }}$. We define the fidelity of $T_{\text {approx }} \equiv T_{\mathrm{a}}$ as the average fidelity of the transmitted fields it predicts, where the average is taken 
over the ensemble of all possible incident waves (or equivalently, over any complete basis of incident modes).

Hence, we find for the overlap between the full and experimental transmission matrix,

$$
\gamma_{\mathrm{TM}}=\frac{\sum_{i, j} T_{\mathrm{t}}^{*}(i, j) T_{\mathrm{a}}(i, j)}{\sqrt{\sum_{i, j}^{N} T_{\mathrm{a}}^{*}(i, j) T_{\mathrm{a}}(i, j)} \sqrt{\sum_{i, j}^{N} T_{\mathrm{t}}^{*}(i, j) T_{\mathrm{t}}(i, j)}} .
$$

We further assume that a priori the transmission matrix elements are independently identically circular-Gaussian distributed (Ginnibre ensemble), and that the approximate matrix $T_{\mathrm{a}}$ is effectively an $n \times m$ matrix, meaning its matrix elements outside this block are zero. As a result we can rewrite the limits of the sum,

$$
\gamma_{\mathrm{TM}}=\frac{\sum_{i, j}^{m, n} T_{\mathrm{t}}^{*}(i, j) T_{\mathrm{a}}(i, j)}{\sqrt{\sum_{i, j}^{m, n} T_{\mathrm{a}}^{*}(i, j) T_{\mathrm{a}}(i, j)} \sqrt{\sum_{i, j}^{N, N} T_{\mathrm{t}}^{*}(i, j) T_{\mathrm{t}}(i, j)}} .
$$

Due to the identical independent distribution of the matrix elements, only the terms where $i=j$ are nonzero after averaging. We assume they are equal to $|s|^{2}$, while all other terms average to zero. As a result,

$$
\left\langle\gamma_{\mathrm{TM}}\right\rangle=\min (m, n)|s|^{2}\left[\min (m, n)|s|^{2}\right]^{-1 / 2}\left[N|s|^{2}\right]^{-1 / 2}
$$

resulting in the expression for the fidelity

$$
F_{\mathrm{TM}} \approx \frac{\min (m, n)}{N}
$$

Hence our estimate for the fidelity of a transmission matrix is the minimum of the fractions $m / N$ and $n / N$, possibly corrected for noise effects. This leads to the surprising conclusion that the largest of the two control fractions apparently does not matter within the validity of this approximation, and to optimize fidelity one should aim for a balanced control on both sides of the medium.

\subsubsection{Fidelity of digital optical phase conjugation}

For digital optical phase conjugation first a transmitted field is measured, and then digitally reproduced and propagated back. We defined the fidelity 
of a digital optical phase conjugation as the average fidelity of phase conjugated field it produces, averaged over the ensemble of waves in a waveguide geometry. analogous to the fidelity of a transmission matrix. This is similar to the measurement of a transmission matrix, except for the back propagation of the transmitted field.

\subsection{Hardware}

The apparatus can be divided in four different modules, each with its own specific functionality. There is a single, tunable laser source that is used as a source. There are two interferometry modules that are used to measure light fields. There are two field synthesis modules that are used to generate arbitrary phase and amplitude holograms. Finally there is a sample holder that can be used to precisely and accurately move the sample that we project light through. In this section we will look at the different modules

\subsubsection{Laser module}

Light is generated by an external cavity diode laser. The laser is coupled to six polarization maintaining fibers to guide the light to the different modules. Half-wave plates and polarizing beamsplitters are used to change the power ratio between the different fibers. A filter wheel with ND-filters is installed to attenuate the power used for the interferometry (Section 2.3.3). The light that is transmitted back through the fibers leading to the wavefront shaping modules can be collected trough flip-mirrors on a photodiode for alignment purposes. The photodiode signal is amplified with a transimpedence amplifier (Femto DLPCA-200). The laser source has a tuning range of 765-781 nm with a tuning resolution of $0.01 \mathrm{~nm}$. It has a linewidth of less than $200 \mathrm{kHz}$ for a $50 \mathrm{~ms}$ integration time [12]. Long-time fluctuations are mainly due to slow temperature changes [13].

\subsubsection{Interferometry}

The goal of the field measurement module is to measure the spatial field profile of a light field. Two of these modules are present in the apparatus, one for each side of the sample. We measure the local phase, and amplitude, for each 


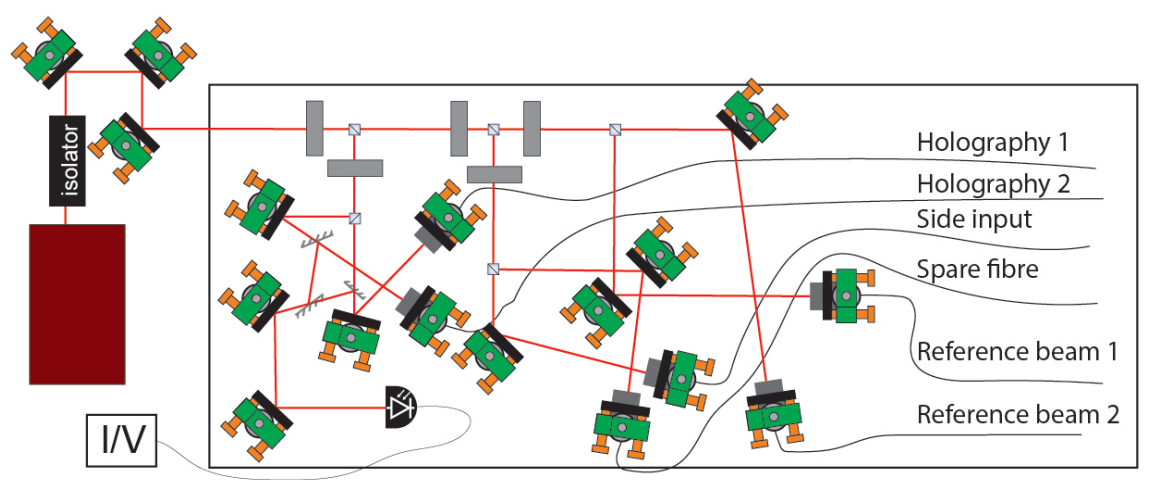

Figure 2.1.: Schematic of the fiber coupling module. Flip mirrors are depicted with transparency. The half-wave plates are indicated as grey rectangles.

polarization of a field with off-axis holography [14]. The total transmitted intensity is measured on a separate camera. In this section we first describe the modules that are used for off-axis holography, then we summarize the signal processing of the holograms.

We depict a schematic of the field measurement module in Fig. 2.2. A 50:50 beamsplitter is used to spatially overlap a diagonally polarized reference beam with the signal beam. The light is split into a horizontal and vertical polarization component with a polarizing beamsplitter (PBS). The signal beam then interferes with the reference beam on CCD1 and CCD2 (AVT Dolphin F145-B). These CCD cameras are monochromatic, with a resolution of $1392 \times 1040$, and a pixel size of $6.45 \times 6.45 \mu \mathrm{m}$.

The total transmitted intensity is measured on CCD3 (AVT Stingray F145B-30fps or AVT Guppy with a resolution of 1038x1388 and 1040x1392, and a pixel size of $6.45 \mu \mathrm{m}$ and $4.65 \mu \mathrm{m}$ respectively). Due to the large angle between the signal beam and the reference beam, the reference beam does not fall on this camera, which allows separation between signal and reference power.

The local phase and amplitude for the two polarization components of the signal beam are calculated from the interference patterns on CCD1 and CCD2. Following the derivation as in [15], omitting the position dependence 


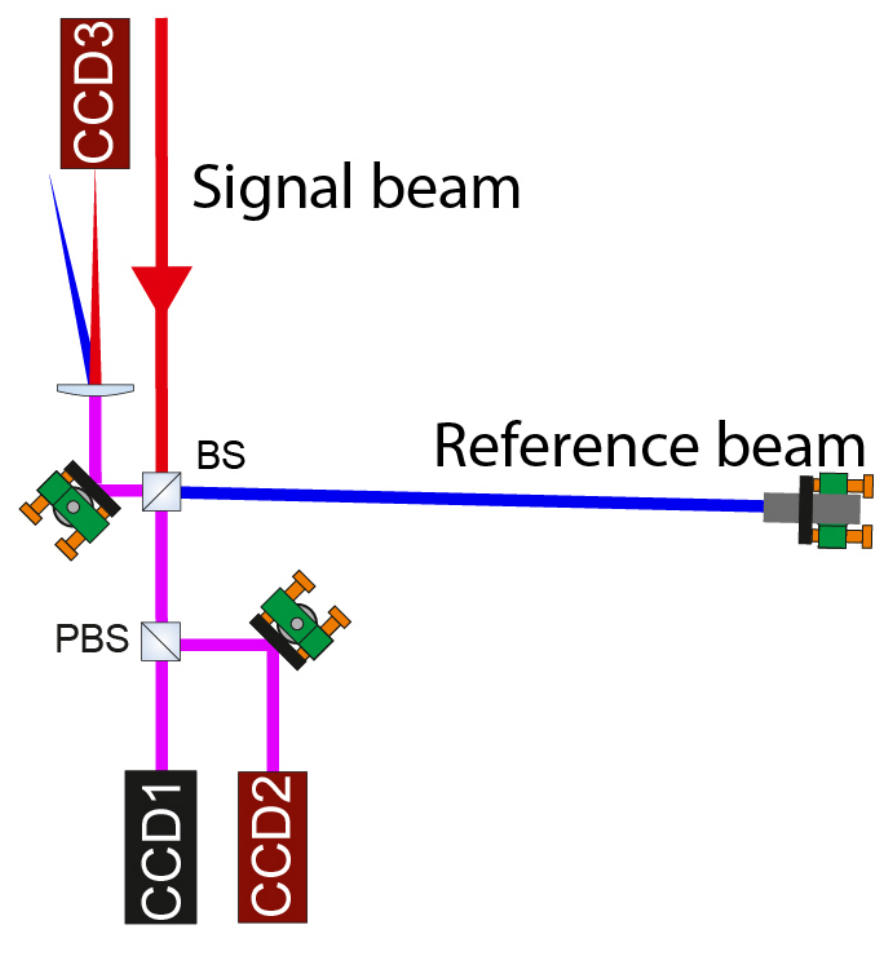

Figure 2.2.: Schematic of the field measurement module. Signal beam in red, reference beam in blue. Overlapped beams in pink. In the experiment the signal and reference beam are from the same source, with the same wavelength.

in the notation, the interference pattern $I$ is described by

$$
\begin{aligned}
I & =\left(E_{\mathrm{s}}+E_{\mathrm{r}}\right)^{*}\left(E_{\mathrm{s}}+E_{\mathrm{r}}\right) \\
& =\left|E_{\mathrm{s}}\right|^{2}+\left|E_{\mathrm{r}}\right|^{2}+E_{\mathrm{s}}^{\uparrow *} E_{\mathrm{r}}+E_{\mathrm{s}} E_{\mathrm{r}}^{\uparrow *} .
\end{aligned}
$$

Where $E_{\mathrm{s}}$ denotes the vertical polarization component of the signal field and $E_{\mathrm{r}}$ indicates the vertical polarization component of the reference beam. The $*$ indicates a complex conjugate. The signal field is defined as

$$
E_{\mathrm{s}}(x, y)=\left|E_{\mathrm{s}}(x, y)\right| \exp \left\{i \phi_{\mathrm{s}}(x, y)\right\},
$$


where $\phi(x, y)$ indicates the local phase for the respective polarization components. Assuming a plane phase front the reference beam can be described as

$$
E_{\mathrm{r}}(x, y)=\left|E_{\mathrm{r}}(x, y)\right| \exp \left\{-i 2 \pi\left(p_{\mathrm{x}} x+p_{\mathrm{y}} y\right)\right\} .
$$

Where $\left(p_{\mathrm{x}}, p_{\mathrm{y}}\right)$ and $\left(q_{\mathrm{x}}, q_{\mathrm{y}}\right)$ are determined by the angle of incidence with respect to the camera. Taking the Fourier transform of Eq. (2.5) we obtain

$$
\mathcal{F}(I)=\mathcal{F}\left\{\left|E_{\mathrm{s}}\right|^{2}+\left|E_{\mathrm{r}}\right|^{2}\right\}+\mathcal{F}\left\{E_{\mathrm{s}}^{\uparrow *} E_{\mathrm{r}}\right\}+\mathcal{F}\left\{E_{\mathrm{s}} E_{\mathrm{r}}^{\uparrow *}\right\} .
$$

The three terms in Eq. (2.8) are separated if $\left(p_{\mathrm{x}}, p_{\mathrm{y}}\right)$ and $\left(q_{\mathrm{x}}, q_{\mathrm{y}}\right)$ are larger than the highest spatial frequency in the signal field. The signal field is calculated from the $\mathcal{F}\left\{E_{\mathrm{s}} E_{\mathrm{r}}^{\uparrow *}\right\}$ component by translating it to $\left(k_{\mathrm{x}}, k_{\mathrm{y}}\right)=$ $(0,0)$ and applying the inverse Fourier transform, which yields

$$
\widetilde{E}_{\mathrm{S}}(x, y)=\left|E_{\mathrm{s}}(x, y) E_{\mathrm{r}}(x, y)\right| \exp \left\{i \phi_{\mathrm{s}}(x, y)\right\}
$$

from which we find the field $E_{\mathrm{s}}(x, y)$ :

$$
E_{\mathrm{S}}(x, y)=\widetilde{E}_{\mathrm{S}}(x, y) / \sqrt{I_{\mathrm{r}}(x, y)} .
$$

For the transmission matrix measurement we slightly modify this procedure, because we sample the fields in the Fourier plane. From Eq (2.8) we isolate the $\mathcal{F}\left\{E_{\mathrm{s}} E_{\mathrm{r}}^{\uparrow *}\right\}$ term. Next we perform a Whittaker-Shannon interpolation by applying an inverse Fourier transform, padding the field with zeros by a factor of five, and applying another Fourier transform. The field is sampled from this interpolated field.

\subsubsection{Field synthesis}

The goal of the field synthesis module is to create a random light field, with a large number of pixels of which the phase and amplitude are controlled. We need a modulation fidelity $F>0.9$, which implies that phase, amplitude and polarization must be modulated. To achieve this, we use a combination of a digital micromirror device and Lee holography [16].

Fields are synthesised with a binary amplitude mask created by a digital micromirror device (DMD). We use a ViALUX V-9600 module with a 0.96" WUXGA resolution DMD chip (Texas Instruments) and a VX4100 control 
board (ViALUX). The DMD has a resolution of $1920 \times 1200$ mirrors with a pitch of $10.8 \mu \mathrm{m}$, can operate at array switching rates of up to $9.7 \mathrm{kHz}[17]$, and has a buffer for 13981 binary frames.

In Fig. 2.3 we depict the field synthesis module. Light is collimated using a fiber collimator (C1, Schäfter+Kirchhoff) into a wide beam $(18 \mathrm{~mm})$ and split into two parallel orthogonal polarization components using a polarizing beamsplitter, a gold coated prism, and two mirrors. The two beams are reflected of two regions on the DMD. The diffracted field is Fourier filtered using a 4 f-configuration and an aperture. The two polarizations are recombined using a similar configuration with a set of mirrors and a polarizing beamsplitter. The DMD is mounted under an angle of $45^{\circ}$ such that the mirrors rotate around an axis that is vertical with respect to the optical table. The modules that split and combine the light in two polarization components are mounted on triangular aluminium blocks such that they are mounted at an angle of $45^{\circ}$. A half wave plate rotates the two diagonal polarization components after the $4 \mathrm{f}$-configuration such that they are horizontally and vertically polarized. CCD1 images the spatial filter in the counter propagating direction using pellicle beamsplitter BS1. A mirror M1 is placed after the half wave plate which reflects the light back. CCD2 is placed such that it images the spatial filter.

We create the arbitrary light field using Lee holography [16], which uses a binary amplitude mask to generate a light field of which both the local amplitude and phase are controlled. We modulate each polarization individually with a separate binary amplitude mask. We use a circular area with a diameter of 636 mirrors per polarization. In Lee holography multiple binary amplitude pixels (mirrors) are used to control a single pixel of the field, which gives an effective resolution of about 4500 independent pixels per polarization. Our implementation of Lee holography enables the use of the superpixel method [18] which has a slightly higher modulation fidelity, but is not implemented because in Lee holography it is easier to adjust the carrier frequency to correct for the dispersion of the DMD.

For the correct operation of the holography the spatial filter has to be aligned exactly. Fig. 2.4 shows the image of the spatial filter on CCD1 and CCD2 after alignment. The outline of the aperture can be seen with a bright spot in the center. Four other dots are visible in the intercardinal directions, corresponding to the highest $k$-vectors that can be synthesised. Fig. 2.4 (a) 


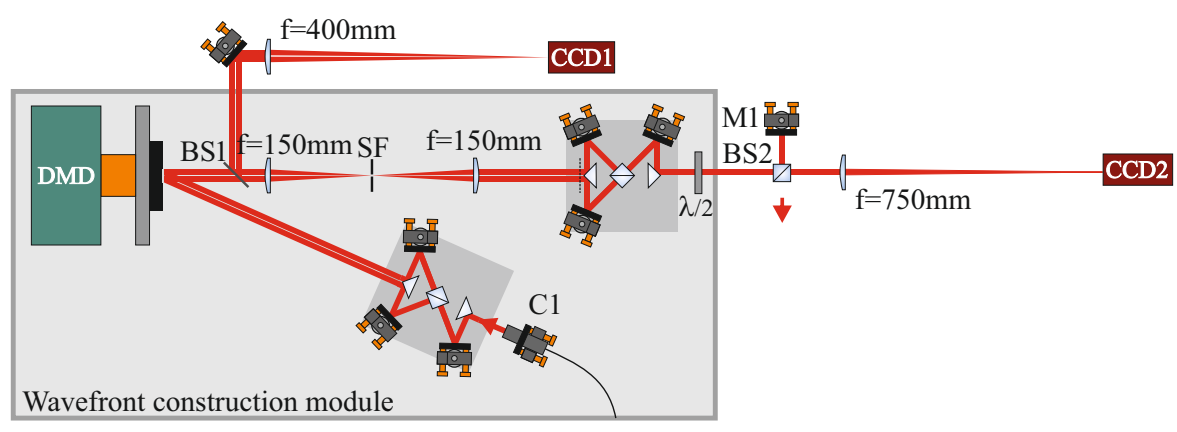

Figure 2.3.: Field synthesis module. Figure adapted from [15]
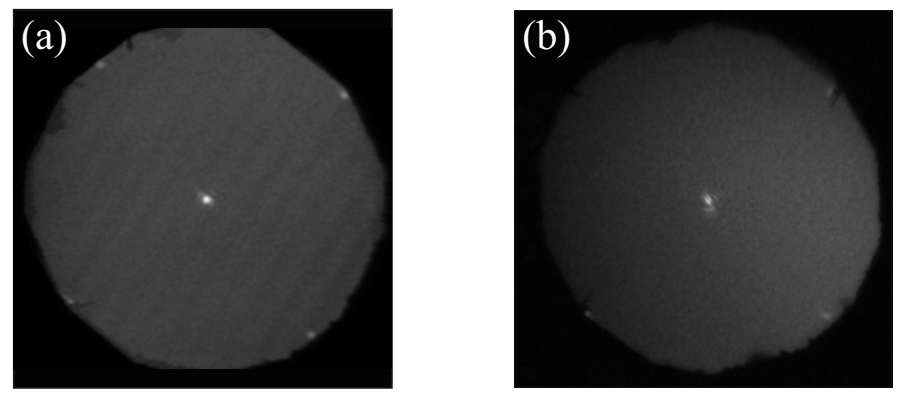

Figure 2.4.: Image of the spatial filter after alignment. The outline of the spatial filter $(\sim 3 \mathrm{~mm})$ is visible.

depict the image on CCD2 and Fig. 2.4 (b) shows the image on CCD1.

\subsubsection{Sample holder}

The sample module projects the light field, generated in the field synthesis module, on the sample, and images the surface of the sample onto the field measurement module. Additionally it allows an accurate and precise displacement of the sample. It uses high numerical aperture objectives in order to collect a large fraction of the power and to achieve a high spatial resolution. The magnification is chosen such that each effective pixel of the field synthesis module is mapped to a spot the size of the diffraction limit on the surface of the sample. 
We show the schematic imaging configuration in Fig. 2.5. A sample is imaged on one side to an image plane, IP1, using a tube lens ( $f=750 \mathrm{~mm}, 2$ " achromat) and a $0.95 \mathrm{NA}, 63 \times$ objective (Zeiss N-Achroplan, 420984-9900, O1). The other side of the sample is imaged to image plane IP2, using a tube lens ( $f=750 \mathrm{~mm}, 2$ " achromat) and a $1.4 \mathrm{NA}, 63 \times$ oil immersion objective (Zeiss Plan-Apochromat, 440760, O2) imaging the sample through the cover glass. The objectives are mounted on closed-loop piezo stages ( $80 \mu \mathrm{m}$ scan range, PiezoJena PX 100-SG) to allow refocussing of the objectives. The piezo stages are mounted on manual 3D stages (Elliot) for longer distance alignment. The sample is mounted on a stage operated by two stickslip linear positioners (SmarAct SLC-1720-S and SLC-1740-S), this allows a manometer precision accurate and repeatable displacement of the sample orthogonal to the optical axis. The inset depicts how the light is focussed on the surface of the sample. It depicts the maximum angle of the incident light, the size of a diffraction limited spot, how the sample is deposited on the cover slide and the size of the controlled area.

The measured magnification between the interferometry mapped to IP2 and IP1 and the sample surface is $288 \mathrm{x}$. We calibrate the magnification with respect to the sample stages by translating the sample by a known distance and measuring the displacement on the camera. With this magnification we have a circular control area and field of view with radius $23 \mu \mathrm{m}$, with a speckle size of $116 \mu \mathrm{m}$ and $79 \mu \mathrm{m}$ in IP1 and IP2 respectively. This corresponds to the effective resolution of the field synthesis module, which has a resolution of approximately $80 \mu \mathrm{m}$. 


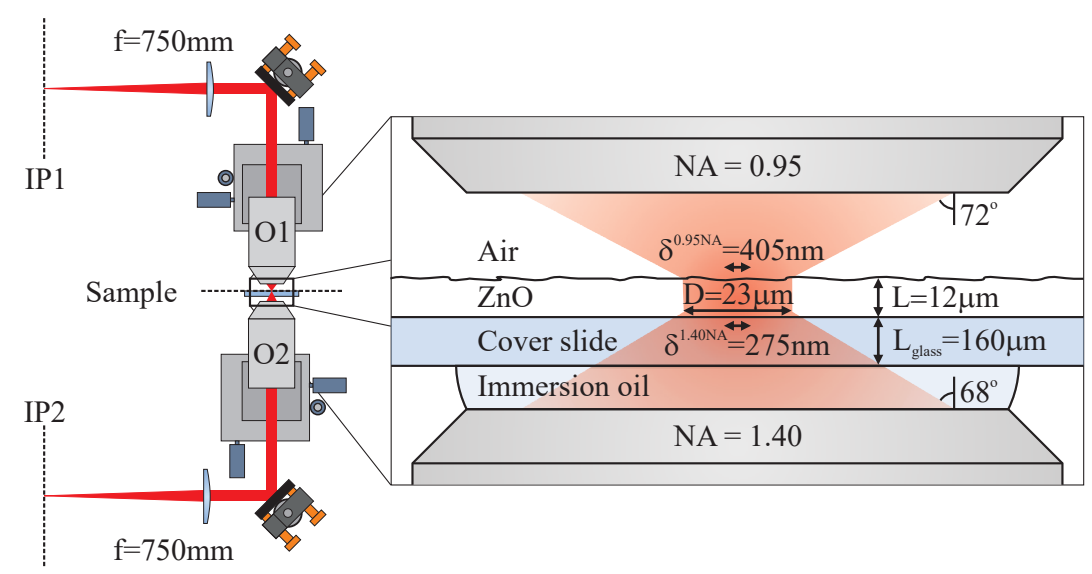

Figure 2.5.: Imaging configuration used. Figure adapted from [15]

\subsection{Sample}

For our experiments we use a strongly scattering material deposited on a glass cover slide, as depicted in Fig. 2.6. The sample has a slight thickness gradient. By selecting a different region of the sample to perform an experiment on we perform measurements with different sample thickness. Part of the cover slide is cleaned from scattering material to allow measurements without a scattering medium present. Our samples have a thickness of up to $20 \mu \mathrm{m}$, and a transport mean free path of $0.7 \mu \mathrm{m}$ [19].

We prepare the samples following a recipe based on van Putten [19]. We use commercially available zinc oxide nanopowder (io-li-tec, Zinc(II)oxide powder, 90-210 nm, 99.9\%). The nanoparticles are suspended in a bottle in water $\left(2.5 \mathrm{~g} \mathrm{ZnO}\right.$ in $8 \mathrm{~g} \mathrm{H}_{2} \mathrm{O}$ ), the mixture is mixed on a rotating vial holder overnight and the bottle is put in an ultrasonic bath to dissolve agglomerates of powder. We clean a microscope slide with ethanol and a plasma cleaner. Then the solution is sprayed on the microscope slide with a commercially available air spray gun (Harder \& Steenbeck, Evolution, $400 \mu \mathrm{m}$ nozzle), and left to dry. After drying we apply the doctor blade technique [20] to create a thickness gradient over the sample, which allows us to measure the transmission matrix at different thicknesses on the same prepared sample. 


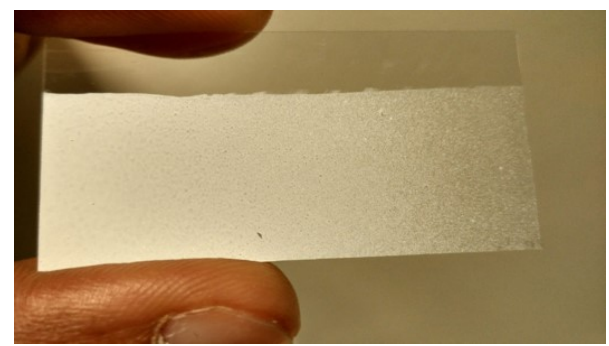

Figure 2.6.: Photograph of scattering sample. The sample is deposited on a coverslide. Part of the coverslide has been cleaned to allow for measurements without a scattering medium present. A thickness gradient is seen along the long direction of the sample.

\subsubsection{Sample thickness}

An in-situ measurement of the local thickness of our sample allows us to accurately know the physical thickness of the sample, even if globally large thickness variations are present. The measurement procedure is as follows: we overlap the focus of our two objectives, by focussing on the same surface of a clean part of the cover slide that the sample is deposited on. Then we shift the sample to the position of the measurement, and refocus on the surface on both sides of the sample. From the net displacement of the objectives we calculate the local thickness of the sample. To focus on the sample we generate temporally and spatially incoherent light with the DMD by creating 160 different random phase patterns, and integrating over these patterns on the camera. This allows us to accurately focus on the surfaces of the sample, with an estimated accuracy of $1 \mu \mathrm{m}$.

\subsection{Physical alignment}

In order to perform phase conjugation with a high fidelity, it is important that all the modules are aligned properly with respect to each other. Different approaches have been taken in order to align a digital phase conjugation system [21-24], in this section we describe our approach to alignment of the phase conjugation system. We physically align the relative position and angle between the different modules. Additionally we have implemented a 


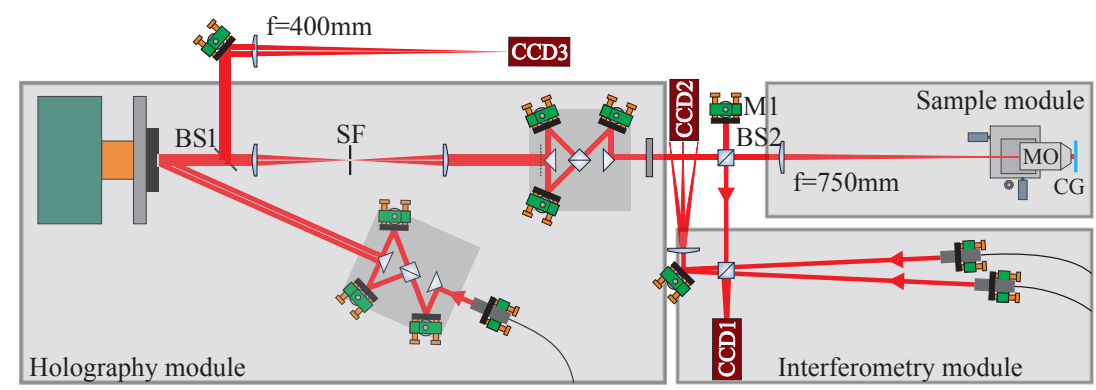

Figure 2.7.: Half of the field conjugation apparatus. Note that the pellicle beamsplitter (BS1) is only used during alignment. Figure adapted from [15].

second alignment procedure in software (Section 2.6), to perform a second higher accuracy alignment.

Fig. 2.7 shows how the three different types of modules are connected to each other. The three different modules are depicted, which are connected using a beamsplitter (BS2).

The physical alignment is composed of two steps: spatial alignment, and angular alignment. We explain how we align along the optical axis and how we correct for rotation, mirroring, and shifts around and orthogonal to the optical axis respectively.

In order to align the image plane of the holography module and the interferometry module along the optical axis we use a multiply scattering material, the sample module and temporary incoherent light. To create temporal incoherent light, we generate 160 fields, with the same amplitude profile, but different random phase profiles. The fields are projected sequentially at 2000 fields per second, generating temporal and spatial incoherent light. The camera exposure time is chosen such that it integrates over a multiple of 160 fields to average over the fluctuations. Next we physically shift both the imaging objective and the camera such that the DMD is properly imaged to the sample and the surface of the sample is imaged to the camera simultaneously. This can be seen in Fig. 2.8. Proper alignment of the sample with respect to the camera and the DMD is indicated by the well defined shape of a square with a hole and simultaneous sharp imaging of the speckle background. 


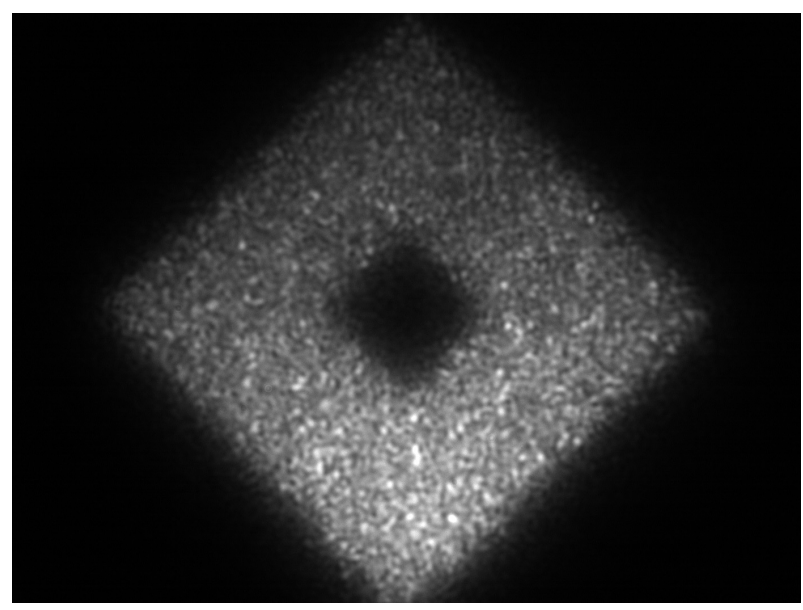

Figure 2.8.: Alignment pattern used for alignment along the optical axis and focusing on the sample.

The objectives and cameras are aligned sequentially with respect to the scratched surface of the cover glass. This ensures both the detection and the synthesis module are sharply imaged to the cover glass.

We also perform an the alignment of the rotation around the optical axis, and in the directions perpendicular to the optical axis. The objective is replaced with a low NA objective (Zeiss A-Plan 441030, $10 \times 0.25 \mathrm{NA}$ ) and the sample is replaced by a mirror. A phase pattern with sharp phase jumps is projected with the DMD and the reflected field is measured. In Fig. 2.9 we depict an example of the feedback signal that is used. The pattern is rotated and mirrored such that the rotation and orientation on the camera match the pattern that is projected. The camera is physically shifted to center the projected field on the camera.

For the physical alignment of the relative angle between the image plane of the measurement module and the field synthesis module we use mirror M1 (Fig. 2.7). The sample module is blocked and a plane wave is projected with the DMD. A plane wave is projected at a zero angle from the holography, and the aperture (SF) is imaged in the back direction. The plane wave is reflected back through the center of the spatial filter (Fig. 2.4 (b)). This ensures mirror M1 is perpendicular to the optical path. The plane wave is then imaged using the interferometry and this angle is set as the zero angle. 


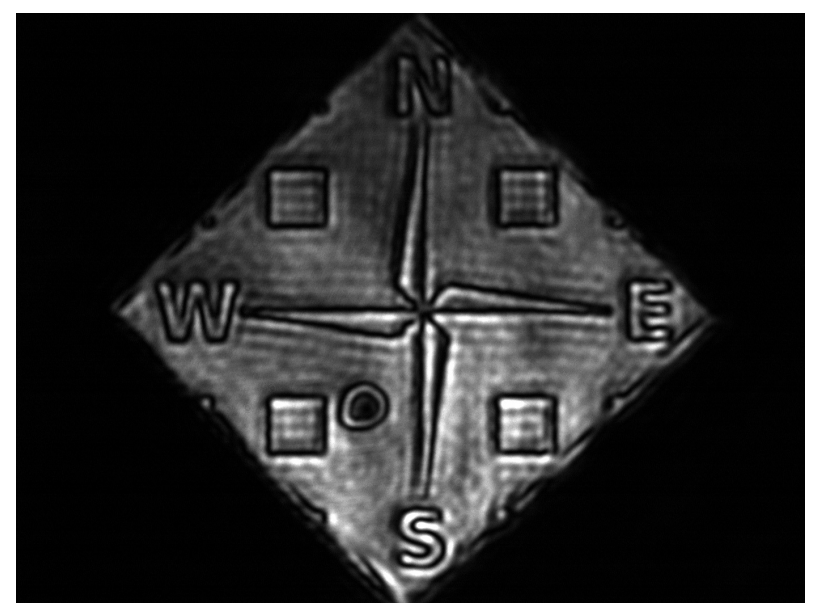

Figure 2.9.: Intensity pattern of alignment field. A dust particle is visible next to the line connecting "W" and "S"

\subsection{Digital aberration corrections}

By performing only a physical alignment, it is typically not feasible to obtain the high accuracy of angular and spatial alignment that is needed for phase conjugation. Additionally there are imaging distortions such as spherical aberrations and coma that are hard to physically correct for. In order to perform alignment at a high accuracy and high precision we perform a second, digital aberration correction. This allows us to correct for the angular and spatial alignment, but also various imaging defects, such as spherical aberrations, coma, and astigmatism. Some of these aberrations might be caused by the 4-f Fourier filter in the field synthesis module, where we have to pass through the lenses off-center in order to create holograms for both polarizations simultaneously. Different methods have been implemented to solve this problem, [21,25-27]. We have developed our own method, tailored specifically to the aberrations and hardware present in our system.

We correct on a Zernike polynomial basis, which is a basis that directly corrects for many common imaging errors and is widely used in adaptive optics [28]. A phase correction with Zernike polynomials is applied in the image plane to correct for errors such as the tilt, spherical aberrations, coma, and astigmatism. We apply corrections for the first 13 Zernike polynomials, 
ordered by their Noll index [29]. After correcting in the real real space, (or, $x, y$ ) a correction in the Fourier plane is performed by applying the same Zernike polynomials with different coefficients. This way we correct for errors such as a shift or a defocus between the interferometry and the holography module. Finally the corrected field is rotated and resampled to the correct resolution to take into account the 45 degree rotation of the DMD and the difference in pixel size between the DMD and the camera. In pseudo-code our correction algorithm is as follows:

$$
\begin{aligned}
& \text { Cut field } E(x, y) \text { to correct size } \\
& \text { Apply Zernike polynomials in image space } \\
& Z_{\text {corr,im }}(x, y)=\Sigma a_{n} Z_{n}(x, y) \text { where } Z_{\text {corr,im }} \\
& \text { denotes the correction, } a_{n} \text { the } \\
& \text { coefficients, and } Z_{n}(x, y) \text { the } \\
& \text { Zernike polynomials. } \\
& E(x, y)_{\text {corrected }}=E(x, y) * \exp \left(i 2 \pi Z_{\text {corr,im }}\right)
\end{aligned}
$$

Fourier transform the field to Fourier space

Apply Zernike polynomials in Fourier space

Fourier transform field back to image space

Perform affine transformation or perspective

transform for imaginary and real part

Rotation

Magnification (resampling)

Apply circular amplitude mask

For alignment we utilize the fact that the correct hologram will both shape a planar wave to the correct speckle pattern, but also shape the correct speckle pattern to a plane wave. This way the correct hologram couples light to the fiber collimator that is normally used to illuminate the DMD. This process is described also in [30] as a single mode reflector.

In Fig. 2.10 we illustrate our experimental procedure. The procedure is the following: light is sent through a scattering medium. We measure the transmitted field on the field measurement device, calculate the corresponding hologram and write the hologram on the DMD. We measure the power that is coupled to the fiber collimator that we normally use to illuminate the DMD. By adjusting the Zernike coefficients and measuring the corresponding coupled power we determine the optimal settings. Once we have the Zernike 


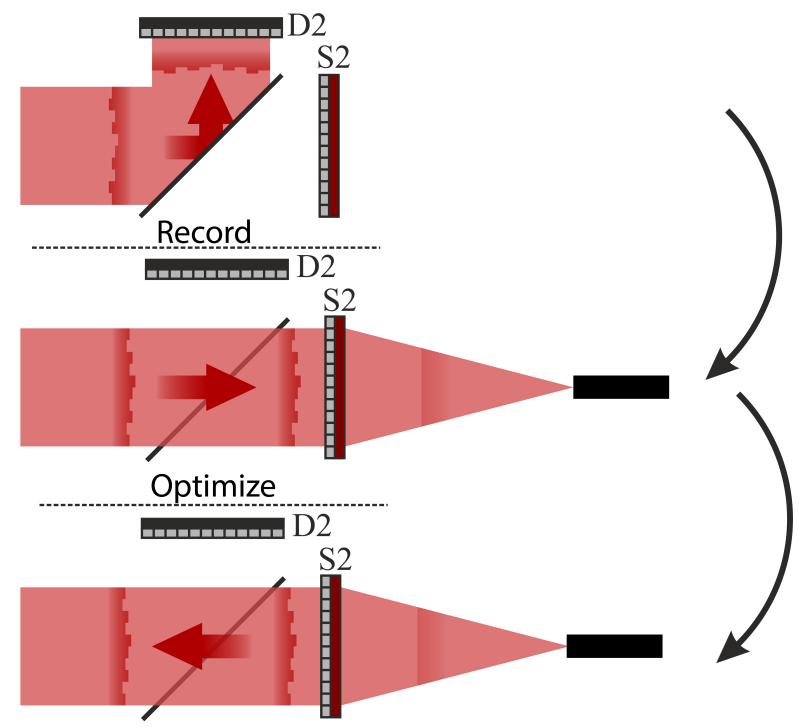

Play back

Figure 2.10.: Schematic representation of the functioning of the SMR phase conjugation. Figure elements adapted from [15].

coefficients we perform a phase conjugation by illuminating the DMD from the fiber collimator. The power coupled to the single mode fiber is linearly proportional to the fidelity, due to the fact that we time-reverse the process by illuminating the DMD from the single mode fiber. This process has a large similarity to the "Single-Mode Reflector" concept described by Lee et al. [30].

Our optimization method also allows us to measure the equivalent of the point spread function (PSF) in the Fourier plane. The hologram is approximately imaged in the Fourier plane on the fiber collimator. By shifting the spot in Fourier space, which we achieve by adding a phase gradient in the image plane - corresponding to Zernike polynomials Z2 and Z3 - we measure the shape of the point spread function. We depict an example of the PSF before and after optimization in Fig. 2.11.

Fig. 2.11 depicts the PSF before (a) and after (b) optimization. The PSF was measured through a clean piece of glass, by phase conjugating a plane wave. We measure transmitted power through the fiber with a software lock- 

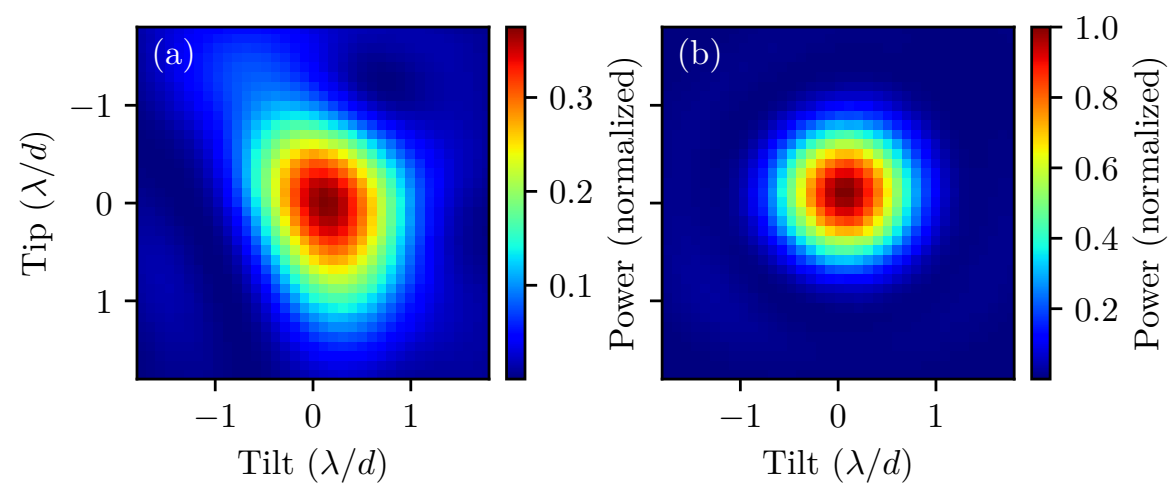

Figure 2.11.: Point spread function before (a) and after (b) optimization. The tip and tilt are measured in units of wavelength per spot diameter $\mathrm{d}$, where $\mathrm{d}$ is the diameter of the phase conjugation area. We normalize (a) and (b) to the maximum value of (b). Note the difference in peak intensity. The PSF was measured through a clean piece of glass, by phase conjugating a plane wave.

in method, for a higher signal to noise, and a transimpedance amplification of $10^{8} \mathrm{~V} / \mathrm{A}$. This high amplification is needed because of the significant loss as the light passes two DMD's in diffraction mode, multiple beamsplitters and couples into the optical fiber twice. The tip and tilt are measured in units of wavelength per spot diameter $d$, where $d$ is the diameter of the phase conjugation area. We normalize (a) and (b) to the maximum value of (b). Note the difference in peak intensity. We clearly see that after optimization the PSF is smaller and radially symmetric. As a result of this the maximum measured intensity, and therefore the fidelity, significantly increases. From the shape of the uncorrected spot, coma and astigmatism can easily be recognized.

The measurement of the fidelity of the phase conjugation on a photodiode provides a direct measurement with a high temporal bandwidth of the fidelity. By measuring a time trace of the fidelity and taking a Fourier transform, we directly measure the spectral noise components. This allows us to identify and address resonances and noise sources in the system. For fi- 
delity measurements we also apply a lock-in type measurement, where we turn the hologram on and off at $2 \mathrm{kHz}$, and select the corresponding Fourier component in the signal.

\subsubsection{Dispersion correction}

To measure wavelength dependent behaviour, we need to correct for any dispersion effects present in the apparatus. This is done in two major ways. First we match the path length of the reference beam with the signal path. This minimizes any phase drift from a wavelength scan. Additionally the beam paths are designed such that the path lengths of the two polarization components match as much as possible, this gives us a limited phase drift between the polarization components when scanning the wavelength.

A strong dispersive effect is present in the field synthesis module. Due to the design of the DMDs it functions as a blazed grating, which induces a strong dispersion, manifested as a phase gradient on the projected field. We correct for the dispersion by adjusting the carrier frequency in the Lee holography, linearly proportional to the laser wavelength. We measure the dispersion with a similar procedure as is used to optimize the Zernike corrections. We illuminate a scattering medium with a collimated beam from a fiber collimator and focus the transmitted light in the single mode fiber. Then we apply a small detuning to the laser, within the Thouless frequency of the scattering material. This will both decorrelate the speckle pattern due the dispersion and due to the change in wavelength. We find the correct dispersion correction by optimizing the dispersion correction applied to the hologram. From our measurements we find that a linear dispersion correction is sufficient to correct dispersion over the full wavelength range.

\subsection{Transmission matrix measurements}

The high degree of control on the incident field, and the high resolution at which we sample the transmitted field allows us to very accurately sample the transmission operator of the sample in a transmission matrix [3,31]. We have implemented a transmission matrix measurement procedure in order to measure transmission matrices. In this section we describe the transmission matrix procedure. 
A transmission matrix relates a set of incident modes on a sample to a set of transmitted mode amplitudes [32]. In this apparatus we map fields from the field synthesis module to fields on the interferometry module. All our transmission matrix measurements are measured with the $0.95 \mathrm{NA}$ air objective for the incident fields, and the transmitted fields are collected with the 1.4NA oil-immersion objective. The fields are sampled in a k-vector basis. As a set of projection fields we use circular aperture plane waves that cover the full control area, of which we vary the k-vector. We sample the transmitted fields on a basis circular plane waves with a size corresponding to the incident control area, which we sample for different $k$-vectors. In order to optimize the sampling density we sample on a hexagonal grid in $k$-space, which allows a higher sampling density than the more commonly used square grid [3]. We match the incident $k$-vectors with the transmitted $k$-vectors from the transmission by measuring the transmission through a cover slide, where we both focus the incident field, and image the transmitted field on the same physical surface.

The spacing of the hexagonal sampling grid is determined by propagating the basis vectors through a scattering sample and varying the incident angle. We calculate the correlation in the propagated field as a function of angular tilt of the incident field, and find the first minimum. We use this angular value as the spacing in the $\mathrm{k}$-vector sampling grid.

The maximum dimension of the measured transmission matrices is determined by the NA and magnification of the objectives, and the stepsize in $k$-space. We sample matrices of $2282 \times 2282$ elements, which covers approximately $80 \%$ of the controlled angles of the incident objective and $38 \%$ of the transmitted modes.

\subsubsection{Matrix normalization}

The transmission matrices are be divided into four different sub-matrices, representing different polarization components in the back focal plane. These components are the horizontal in-horizontal out (hh), vertical in-horizontal out (vh) and correspondingly the hv and vv components. We normalize the hh and vv sub-matrices to the rms-value of the singular vectors of a transmission matrix measurement through a cover slide without sample present. This corrects for a difference in reference intensity and input power for the 
hh and vv components. We normalize the rms value of the sub-matrices to equal the mean rms value of the hh and vv sub-matrices, because we do not directly measure the reference intensity or input power separately. This normalization is correct for full polarization mixing through the sample, for very thin samples it shows a slight overestimation of the hv and vh components which have no ballistic light components.

\subsection{Phase noise in transmission matrix measurement}

We observe a slow phase drift of the absolute phase between the signal beam and the reference beam. Additionally we observe a random phase noise during the transmission matrix measurements.

To correct for the slow phase drift, we take one reference image every five sampling points. We calculate the phase drift for each reference frame by calculating the correlation with the first reference field. We fit a univariate spline to these measured values and interpolate the phase shift for the intermediate points. We use this fit to correct for the phase drift in the different elements of the transmission matrix.

We quantify the random phase noise in the transmission matrix measurements, using a novel measurement procedure. We measure two independent matrices of the same sample in succession and calculate the eigenvalues of $T_{1}^{\dagger} T_{2}$. If the matrices are exactly the same, all eigenvalues will be positive and real, and represent the square of the singular values of the transmission channels. If a small phase noise is present the eigenvalues will become complex. The shape of the eigenvalue scatter plot in the complex plane is dependent on the amount of phase noise in the transmission matrices. We parametrize the shape of the eigenvalue scatter plot according to the following procedure:

$$
\begin{aligned}
& \text { Take two transmission matrices: } T_{1}, T_{2} \\
& \text { Calculate the eigenvalues } w \text { of } T_{1}^{\dagger} T_{2} \\
& \text { Divide the eigenvalues } w \text { in } 30 \text { equal sized } \\
& \text { bins, according to their real part } \\
& \text { Approximate the distribution with a curve: for } \\
& \text { every bin, calculate the standard }
\end{aligned}
$$




$$
\text { deviation on the imaginary axis }
$$

To estimate the phase noise present in $T_{1}$ and $T_{2}$, we follow the following procedure:

$$
\begin{aligned}
& \text { Take one of the matrices: } T_{1} \\
& \text { Add Gaussian phase noise with different } \\
& \text { standard deviations to the columns of the } \\
& \text { matrix: } T_{1}^{\prime \dagger} \text { and } T_{1}^{\prime \prime} \text {. } \\
& \text { For every amount of Gaussian noise calculate } \\
& \text { the shape of the eigenvalue scatter plot, } \\
& \text { Calculate the mean square difference } \chi \text { with } \\
& \text { the curve for } T_{1}^{\dagger} T_{2} \\
& \text { Estimate the phase noise as the value for } \\
& \text { which we get the lowest } \chi
\end{aligned}
$$

By applying this method we find that the phase noise is best described with a standard deviation of 0.185 radian, equivalent to a phase noise of $\lambda / 30$.

\subsection{Wavelength dependent phase drift correction}

The light transmitted through the scattering material will incur a phase delay, associated to the path length of the light inside the sample. However, there is also a phase delay associated with the path length through the system, for both the signal and the reference path. Therefore, in order to measure the absolute phase delay through only the sample we have to match the path length of the reference light with the light transmitted through the sample within a micrometer, because our samples are only several micrometers thick. Experimentally this is possible, however it is not practical and hard to achieve. Experimentally we have matched the pathlength of the reference and the signal path within millimetres. This allows us to only measure the relative phase delay between different transmitted fields, and not the absolute phase delay of any field.

The relative phase between the different polarization components is essential for a correct transmission matrix measurement. Due to small path length differences between the different polarization components phase shifts 
between these components occur when changing the wavelength of the light. We have developed and implemented a simple yet very effective method to correct for these phase drifts. We correct the phase shifts in a way similar to the noise characterization method described in Section 2.8. In order to correct the phase shift, first we calculate the eigenvalues $w=\operatorname{eig}\left(h h_{\lambda}^{\dagger} h h_{\lambda+\Delta \lambda}\right)$, where $h h$ corresponds to one of the submatrices. Next we calculate the argument $\phi_{\text {eig }}$ of the mean of the eigenvalues. Then we rotate one matrix such that the mean of the eigenvalues is real and positive: $h h_{\lambda+\Delta \lambda}^{\prime}=$ $h h_{\lambda+\Delta \lambda} \exp \left\{i \phi_{\text {eig }}\right\}$. We correct for the phase dispersion between spectrally adjacent matrices, i.e. if we measure a transmission matrix at $771 \mathrm{~nm}, 772 \mathrm{~nm}$ and $773 \mathrm{~nm}$, we correct the phase dispersion between $771 \mathrm{~nm}$ and $772 \mathrm{~nm}$, and then the phase drift between $772 \mathrm{~nm}$ and $773 \mathrm{~nm}$.

In Fig. 2.12, we plot the eigenvalues $w$ for the different sub matrices of two transmission matrices before correcting for the path length difference and the sub matrix intensity. We find that the different transmission matrix components hh, hv, vh and vv have different dispersions. 


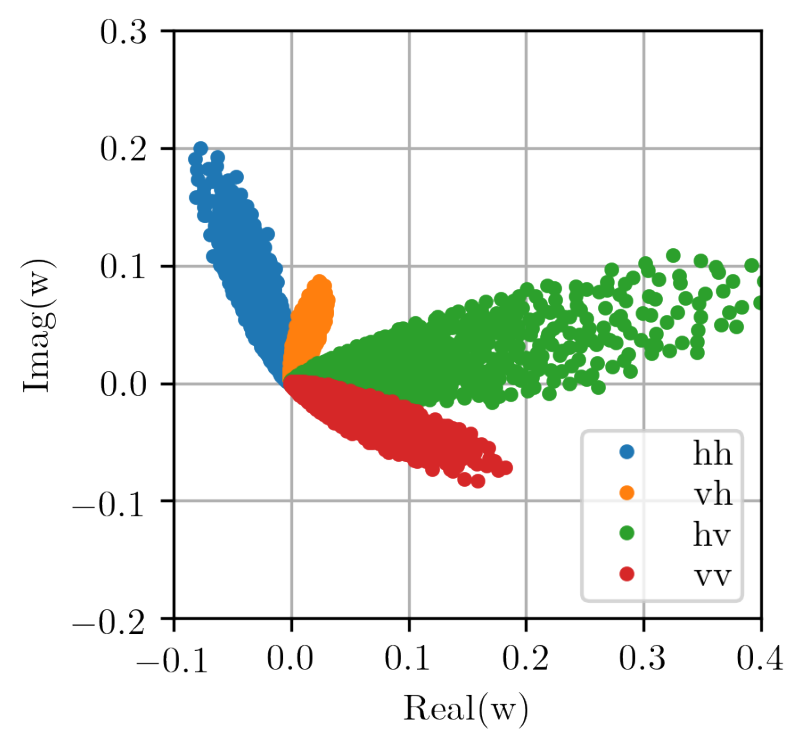

Figure 2.12.: Eigenvalues of $w$ for the different transmission matrix components. From the different orientations of the different components we find that the dispersion of the different polarizations in the measurement apparatus is unequal. The difference in amplitude for the vh and hv components is corrected for in a different correction.

\subsection{Fidelity estimate}

We have found the best estimate for the fidelity of a transmission matrix is the minimum of the fractions $m / N$ and $n / N$, possibly corrected for noise effects. We leave out this noise correction because the phase noise is of the level of $\lambda / 30$ which is only very minor effect compared to the aperture effects. In our experiment, the control fraction is dominated by numerical aperture effects. Within the effective NA of 0.95 of an objective the best value of $m / N$ that can be reached is about $\mathrm{NA}^{2} \approx 0.8$. On the transmission side we have an oil immersion objective, but we sample up to an NA of about 0.9 to obtain a symmetric matrix. This leads to an effective $n / N$ of 0.36 , which is therefore our best estimate of the fidelity in our experiment. The fidelity of the wavefront synthesis may be lower due to an amplitude 
imbalance between different $k$-vectors and polarization components, which are corrected for more easily in the transmission matrix measurements.

\subsection{Conclusion}

In summary, we have introduced an apparatus to observe correlations due to mesoscopic physics in a multiply scattering medium. We have introduced a measure for the fidelity of our apparatus that determines how well we are able to resolve transmission properties of a sample, and which states that a balanced control between the incident and transmitted side of the sample is required. We have introduced the different modules of the apparatus, their individual functions and the alignment procedure. With a Zernike-polynomial based digital aberration correction procedure we correct for optical aberrations in the field synthesis and increase the phase conjugation fidelity. In order to sample the transmission operator of the sample we introduce a transmission matrix measurement procedure and a method to quantify the phase noise which we determine to correspond to $\frac{\lambda}{30}$. Finally we have introduced a method that allows us to measure a multispectral transmission matrix by correcting for phase dispersion between measurements at different wavelengths. 


\section{Bibliography}

[1] D. Andreoli, G. Volpe, S. Popoff, O. Katz, S. Grésillon, and S. Gigan, "Deterministic control of broadband light through a multiply scattering medium via the multispectral transmission matrix," Sci. Rep. 5, 10347 (2015).

[2] M. Mounaix, H. Defienne, and S. Gigan, "Deterministic light focusing in space and time through multiple scattering media with a time-resolved transmission matrix approach," Phys. Rev. A 94, 041802 (2016).

[3] P. Pai, J. Bosch, and A. P. Mosk, "Optical transmission matrix measurement sampled on a dense hexagonal lattice," OSA Continuum 3, 637-648 (2020).

[4] O. N. Dorokhov, "On the coexistence of localized and extended electronic states in the metallic phase," Solid State Commun. 51, 381-384 (1984).

[5] P. A. Mello, P. Pereyra, and N. Kumar, "Macroscopic approach to multichannel disordered conductors," Ann. of Phys. 181, 290-317 (1988).

[6] S. Rotter and S. Gigan, "Light fields in complex media: Mesoscopic scattering meets wave control," Rev. Mod. Phys. 89, 015005 (2017).

[7] V. A. Marčenko and L. A. Pastur, "Distribution of eigenvalues for some sets of random matrices," Math. USSR Sb. 1, 457-483 (1967).

[8] A. Goetschy and A. D. Stone, "Filtering random matrices: The effect of incomplete channel control in multiple scattering," Phys. Rev. Lett. 111, 063901 (2013).

[9] I. M. Vellekoop and A. P. Mosk, "Universal optimal transmission of light through disordered materials," Phys. Rev. Lett. 101, 120601 (2008).

[10] A. P. Mosk, A. Lagendijk, G. Lerosey, and M. Fink, "Controlling waves in space and time for imaging and focusing in complex media," Nat. Photonics 6, 283 (2012).

[11] H. Yilmaz, W. L. Vos, and A. P. Mosk, "Optimal control of light propagation through multiple-scattering media in the presence of noise," Biomed. Opt. Express 4, 1759-1768 (2013).

[12] Velocity $6700 \quad$ Widely Tunable Lasers, Datasheet. Http://assets.newport.com/webDocumentsEN/images/DS_041104_Velocity_Datasheet.pdf, accessed on September 8,2015 . 
[13] New Focus tunable laser linewidth, technical note (2013). Http://assets.newport.com/webDocuments-EN/images/31626.pdf, accessed on September 8, 2015.

[14] M. Takeda, H. Ina, and S. Kobayashi, "Fourier-transform method of fringe-pattern analysis for computer-based topography and interferometry," J. Opt. Soc. Am. 72, 156-160 (1982).

[15] S. A. Goorden, "Reversible scattering of light exploited for quantumsecure authentication," PhD thesis, Universiteit Twente, Enschede (2015).

[16] W.-H. Lee, "Binary synthetic holograms," Appl. Opt. 13, 1677-1682 (1974).

[17] DLP@ V-Modules (2012). Http://www.vialux.de/pdf/vmodules_en.pdf, accessed on September 8, 2015.

[18] S. A. Goorden, J. Bertolotti, and A. P. Mosk, "Superpixel-based spatial amplitude and phase modulation using a digital micromirror device," Opt. Express 22, 17999-18009 (2014).

[19] E. G. van Putten, "Disorder-enhanced imaging with spatially controlled light," PhD thesis, Universiteit Twente, Enschede (2011).

[20] H. Yang and P. Jiang, "Large-scale colloidal self-assembly by doctor blade coating," Langmuir 26, 13173-13182 (2010).

[21] M. Cui and C. Yang, "Implementation of a digital optical phase conjugation system and its application to study the robustness of turbidity suppression by phase conjugation," Opt. Express 18, 3444-3455 (2010).

[22] I. N. Papadopoulos, S. Farahi, C. Moser, and D. Psaltis, "Focusing and scanning light through a multimode optical fiber using digital phase conjugation," Opt. Express 20, 10583-10590 (2012).

[23] T. R. Hillman, T. Yamauchi, W. Choi, R. R. Dasari, M. S. Feld, Y. Park, and Z. Yaqoob, "Digital optical phase conjugation for delivering twodimensional images through turbid media," Sci. Rep. 3, 1909 (2013).

[24] Y. Shen, Y. Liu, C. Ma, and L. V. Wang, "Focusing light through scattering media by full-polarization digital optical phase conjugation," Opt. Lett. 41, 1130-1133 (2016).

[25] M. Jang, H. Ruan, H. Zhou, B. Judkewitz, and C. Yang, "Method for auto-alignment of digital optical phase conjugation systems based on digital propagation," Opt. Express 22, 14054-14071 (2014).

[26] D. Wang, E. H. Zhou, J. Brake, H. Ruan, M. Jang, and C. Yang, "Fo- 
cusing through dynamic tissue with millisecond digital optical phase conjugation," Optica 2, 728-735 (2015).

[27] M. Azimipour, F. Atry, and R. Pashaie, "Calibration of digital optical phase conjugation setups based on orthonormal rectangular polynomials," Appl. Opt. 55, 2873-2880 (2016).

[28] R. K. Tyson, Principles of Adaptive Optics (Academic Press, New York, 1998), 2nd ed.

[29] R. J. Noll, "Zernike polynomials and atmospheric turbulence*," J. Opt. Soc. Am. 66, 207-211 (1976).

[30] K. Lee, J. Lee, J.-H. Park, J.-H. Park, and Y. Park, "One-wave optical phase conjugation mirror by actively coupling arbitrary light fields into a single-mode reflector," Phys. Rev. Lett. 115, 153902 (2015).

[31] S. Popoff, G. Lerosey, R. Carminati, M. Fink, A. Boccara, and S. Gigan, "Measuring the transmission matrix in optics: An approach to the study and control of light propagation in disordered media," Phys. Rev. Lett. 104, 100601 (2010).

[32] M. Nieto-Vesperinas, Scattering and Diffraction in Physical Optics, 2nd Edition (World Scientific Publishing Company, 2006). 


\section{Coupling light to transmission channels}

\subsection{Introduction}

Mesoscopic transport theory is a well established theory, applicable to both light in diffusive media, and electrons in disordered conductors [1-4]. Most of the results describe the ensemble average or correlation functions, as these were experimentally the only accessible functions until recently. Many different effects can be explained, such as enhanced backscattering [5-7], and universal conductance fluctuations [8].

In optics, it is possible to address specific modes of a scattering medium. The work by Vellekoop [9] demonstrates control of the light that is transmitted through a scattering medium and bring light to a focus in transmission by the method of shaping the incident wavefront. Other goals that can be achieved by shaping the incident light are to increase the total transmission of light through the material [10-16] by coupling more light to the open transmission channels, to focus light in time [17-21], to program arbitrary beamsplitters [22-24], and propagate quantum states [24-26]. Acoustic transmission matrices have been measured for a multiple scattering medium [27] followed by transmission matrix measurements for light in scattering media [28-30] which were used to reconstruct images [31]. In optics, all measured transmission matrices have a fidelity lower than 1 due to limited optical access, imperfect sampling and noise. As a result the transmission channels calculated from measured transmission matrices are an approximation of the true transmission channels of the sample ${ }^{1}$.

In this chapter, we experimentally identify and address transmission chan-

\footnotetext{
${ }^{1}$ For brevity, we always refer to the channels found from a measured transmission matrix as channels.
} 
nels for light in strongly scattering media and measure their transmittance as well as reflectance. We find the transmission channels from measured transmission matrices and project the associated light fields using a field synthesizer that controls amplitude, phase and polarization of the field. The transmittance of a transmission channel in the ideal case is given by its transmission eigenvalue, which is the square of the associated singular value of the transmission matrix. However, in the presence of noise, sampling artifacts or other measurement artifacts, the distribution of singular values may be very different from that of actual transmittances. Therefore, we demonstrate two methods to measure transmittance of a transmission channel independent of the singular value of the transmission channels. The first method is to synthesize the field corresponding to the channel and project it through the sample while measuring the transmitted and reflected power over the full numerical aperture. This method is very robust and can be used to verify energy conservation, but is limited by the fidelity of the wavefront synthesis which is lower than that of measuring transmission matrix. The second method is to calculate transmittance by digitally propagating the field through an independently measured transmission matrix of the same sample. The advantages of this method are first it can be performed in post processing as long as at least two independently measured matrices are available, and second that it has the high fidelity of the transmission matrix measurement. As a disadvantage, it is sensitive to systematic sampling effects that occur in both measured matrices. These sampling effects are minimized in our measurement procedure [32]. We demonstrate that transmittances measured by this method correspond well to the singular values of the matrix, underlining the high quality of our transmission matrix data.

\subsection{Transmission matrix measurement}

To measure the transmission matrix $T$, we use the procedure as described in Chapter 2. In this section we briefly reiterate the configuration used for the transmission matrix measurement and depict the singular value histograms corresponding to our transmission matrices.

In order to find high transmittance transmission channels, we sample the field over a large field of view, and over large angles. We project the in- 

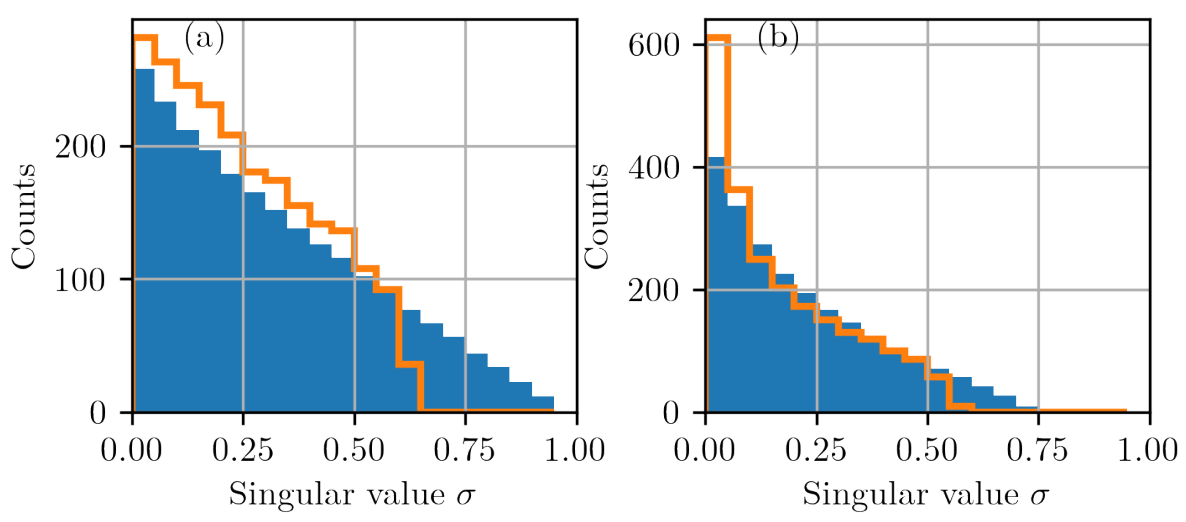

Figure 3.1.: Singular value histogram of the transmission matrix of the

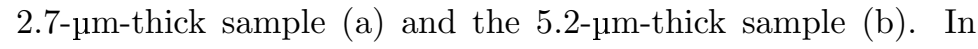
orange the histogram for a filtered DMPK model according to Pai [34], with no free parameters.

cident fields with a high-NA objective (0.95NA, 63×, Zeiss N-Achroplan, 420984-9900) and collect the transmitted light with a high NA oil immersion objective (1.4NA, 63×, Zeiss Plan-Apochromat, 440760). We sample a transmission matrix at 2282 points in $k$-space, sampling the transmitted and projected field up to an NA of approximately 0.9. The measurements reported in this chapter are performed on two $\mathrm{ZnO}$ samples, with thicknesses of $2.7 \mu \mathrm{m}$ and $5.2 \mu \mathrm{m}$, transmittances of $0.45 \pm 0.05$ and $0.26 \pm 0.03$ respectively and the same scattering mean free path $l \approx 0.7 \mu \mathrm{m}[33]$.

We depict a histogram of the the singular values $\sigma$ of the transmission

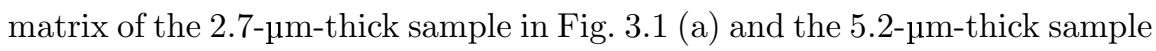
in Fig. 3.1 (b). We find a value of $\sigma / \sigma_{\mathrm{rms}}$ of 2.44 for the thinner sample and 2.59 for the thicker sample. In orange we fit the histogram of a simple filtered DMPK model, calculated according to Pai [34]. The model has no adjustable parameters, its fixed parameters are a sampling fraction of 0.36 , and the internal sample transmittance which differs from the measured value by a factor 0.58 which corrects for extrapolation effects on the air side. We see a good agreement between the data and the model, and a strong deviation from the quarter circle distribution that is predicted by Marchenko and Pastur for an uncorrelated matrix [35]. 


\subsection{Coupling light to transmission channels}

In this section, we find the transmission channels of a multiple scattering material and couple light into them. We define the physical transmittance $\tilde{T}^{(n)}$ and physical reflectance $\tilde{R}^{(n)}$ of transmission channel $(n)$, that we measure by physically propagating the transmission channel through the sample and collecting the transmitted and reflected light. We show that by projecting the singular vectors we can either enhance or suppress the transmitted light and that the physical transmittance and reflectance add up to 1 for all channel.

We find the transmission channel $\boldsymbol{v}^{(n)}$ from the $n$th column of the left singular matrix of the transmission matrix $T$ by performing a singular value decomposition $T=U \Sigma V^{\dagger}$, where $U$ and $V$ are unitary matrices and $\Sigma$ is a positive diagonal matrix containing the singular values. After calculating the transmission channels $\boldsymbol{v}^{(n)}$ we calculate the corresponding holograms, and project the singular vectors through the scattering material. To calibrate the transmittance and the reflectance we perform three measurements. We measure the transmitted and reflected power for the singular vectors through a clean part of the cover slide $\left(P_{g, T}^{(n)}, P_{g, R}^{(n)}\right)$, through the part of the sample for which we measured the transmission matrix $\left(P_{s, T}^{(n)}, P_{s, R}^{(n)}\right)$, and through an uncorrelated part of the sample $\left(P_{r, T}^{(n)}, P_{r, R}^{(n)}\right)$. The uncorrelated measurement is taken at a position shifted $1.4 \mu \mathrm{m}$ from where the transmission matrix was measured. This distance is large enough such that the transmission matrix is fully decorrelated while maintaining the same sample thickness. We measure the transmitted power and the reflected power on cameras which image the back focal aperture of the objectives. Additionally to the transmitted power we also measure the transmitted fields. These measurements allow us to calculate the increase in total transmittance and reflectance, both for the subspace of controlled $k$-vectors, and for all the collected light within the full NA of the objective. We take background measurements to correct for spurious reflections of the reference beam. Note that for the results described in this section the projected transmission channels are calculated in real time during the experiment from a transmission matrix for which we have not normalized the submatrices (Section 2.7.1). This reduces the effective fidelity of the transmission matrix and thereby the maximal achievable transmittance. This lack of normalization does not change the measured transmitted or re- 
flected intensities, as these are measured in an independent way. In later sections, post-processed transmission matrices are used in which we correct for this measurement error, as described in Section 2.7.1.

Due to the limited numerical aperture of the objectives we do not capture all the transmitted or reflected intensity. In transmission and in reflection we capture the light within a $69^{\circ}$ resp. $72^{\circ}$ angle. For a Lambertian escape function, this means we collect $90 \%$ of the emitted light in both cases. We observe that the escape function drops below the Lambertian curve at high angles as expected from escape function theory [36]. Since the Lambertian approximation overestimates the escape function we conclude that we collect more than $90 \%$ of the transmitted light.

We are interested in the physical transmittance and reflectance for each incident channel. Conceptually, one would measure these by dividing the transmitted (reflected) flux by that measured on a perfectly transmitting (reflecting) sample for the same incident wave. However that measurement is not practical as it entails changing or translating the sample between reference and signal measurements, leading to possible systematic errors. Therefore we define experimental approximations of the transmittance $\tilde{T}^{(n)}$ and reflectance $\tilde{R}^{(n)}$ as $\tilde{T}^{(n)}=\left(P_{s, T}^{(n)} / P_{r, T}^{(n)}\right)\left\langle P_{r, T}^{(n)}\right\rangle /\left\langle P_{g, T}^{(n)}\right\rangle$ and similarly the reflectance $\tilde{R}^{(n)}=\left(P_{s, R}^{(n)} / P_{r, R}^{(n)}\right)\left(1-\left\langle P_{r, T}^{(n)}\right\rangle /\left\langle P_{g, T}^{(n)}\right\rangle\right)$, where \langle\rangle indicates averaging over all $n$ channels.

Our experimental approximations are defined in terms of precisely measurable quantities, namely the transmittance increase $\eta_{T, \text { total }}^{(n)}=P_{s, T}^{(n)} / P_{r, T}^{(n)}$ and the reflectance increase $\eta_{R \text {,total }}^{(n)}=P_{s, R}^{(n)} / P_{r, R}^{(n)}$. We calculate the transmittance and reflectance by multiplying the average transmittance with the transmission increase, and the average reflectance with the reflectance increase, $\tilde{T}^{(n)}=\eta_{T, \text { total }}^{(n)} \tilde{T}_{\text {avg }}$ and $\tilde{R}^{(n)}=\eta_{R \text {,total }}^{(n)}\left(1-\tilde{T}_{\text {avg }}\right)$. Here $\tilde{T}_{\text {avg }}$ corresponds to the average transmittance $\tilde{T}_{\mathrm{avg}}=\left\langle\tilde{T}^{(n)}\right\rangle=\left\langle P_{r, T}^{(n)}\right\rangle /\left\langle P_{g, T}^{(n)}\right\rangle$, where \langle\rangle denotes averaging over all channels.

To measure the physical transmittance of each channel we propagate the transmission channels through a strongly scattering medium with average transmittance $\tilde{T}_{\text {avg }}=0.26 \pm 0.03$. We also calculate $\eta_{t, \text { total }}^{(n)}$ and $\eta_{r \text {,total }}^{(n)}$, in Fig. 3.2 (a) we see that we increase the transmitted power by up to $89 \%$, while suppressing the reflected power by $25 \%$. In Fig. 3.2 (b) we plot the reflectance $\tilde{R}^{(n)}$ and physical transmittance $\tilde{T}^{(n)}$. We find a transmittance 

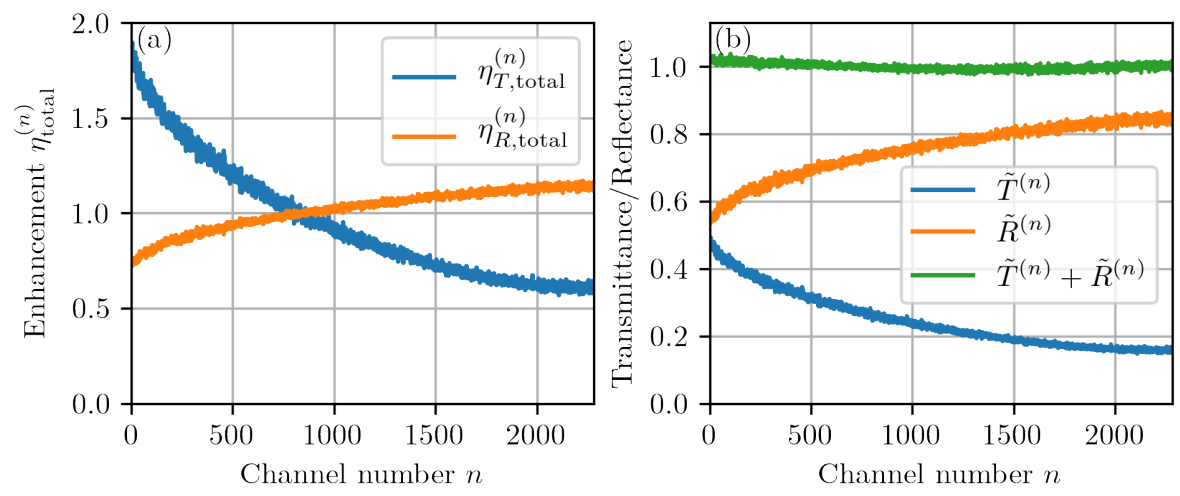

Figure 3.2.: Transmittance increase $\eta_{T \text {,total }}^{(n)}$ and reflectance increase $\eta_{R \text {,total }}^{(n)}$ versus channel number (a). In (b) we depict the reflectance $\tilde{R}^{(n)}$ and physical transmittance $\tilde{T}^{(n)}$. The data is for the $5.2 \mu \mathrm{m}$ sample.

$\tilde{T}^{(n)}$ between 0.14 and 0.475 . In the definition of $\tilde{R}^{(n)}$ and $\tilde{T}^{(n)}$ we assume that we capture all the transmitted and reflected light, while experimentally we capture approximately more than $90 \%$ but not $100 \%$ of the light. We find that the transmittance $\tilde{T}^{(n)}$ and reflectance $\tilde{R}^{(n)}$ add up to 1 for all channels. This demonstrates that we capture the same fraction of the light in both transmission and reflection, not only on average but for each channel separately. In earlier work, Kim and Choi [14,15] show simultaneous measurements of the transmittance and reflectance of the projected reflection channels, where the transmittance and reflectance do not add up to 1 for all channels. The loss of light measured by Kim and Choi is likely due to light leakage to outside of the numerical aperture [37].

We also look at a second, thin $(2.7 \mu \mathrm{m})$ sample with $\tilde{T}_{\text {avg }}=0.45 \pm 0.05$. For this sample we can increase the physical transmittance such that more light is transmitted than reflected and vice versa. In Fig. 3.3 we plot the transmission data as a function of channel number. In (a) we plot $\eta_{T \text {,total }}$ and $\eta_{R \text {,total }}^{(n)}$ versus channel number. In (b) we plot the transmittance $\tilde{T}^{(n)}$ and reflectance $\tilde{R}^{(n)}$. We find that the reflectance $\tilde{R}^{(n)}$ and physical transmittance $\tilde{T}^{(n)}$ add up to 1 for all channels, and that a significant fraction of the transmission channels transmit more light than they reflect. We measure a maximum 

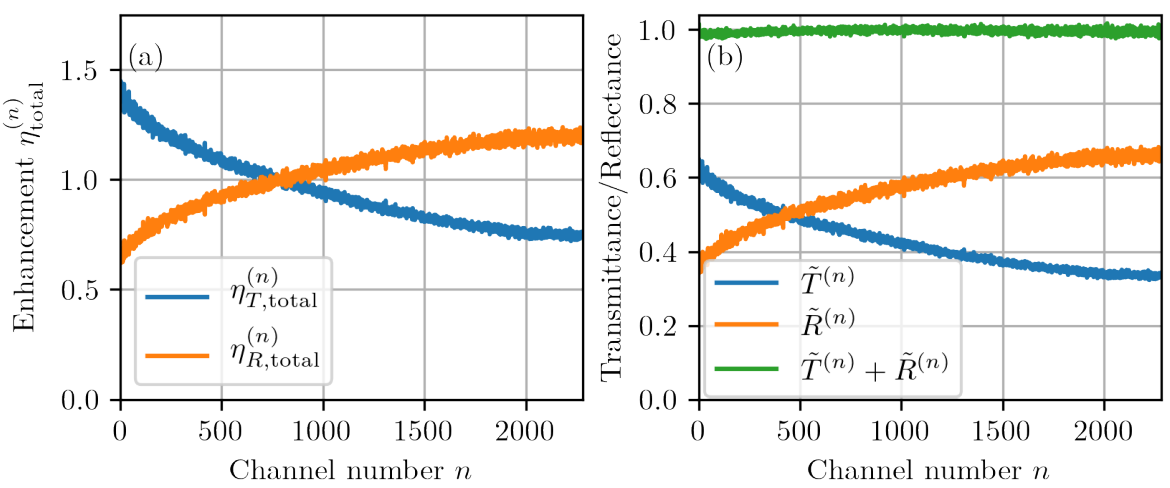

Figure 3.3.: Transmittance $\eta_{T \text {,total }}^{(n)}$ and reflectance $\eta_{R \text {,total }}^{(n)}$ enhancement versus channel number for a thin $(2.7 \mu \mathrm{m})$ sample with an average transmittance $\tilde{T}_{\text {avg }}=0.45(\mathrm{a})$. In (b) we plot the reflectance $\tilde{R}^{(n)}$ and physical transmittance $\tilde{T}^{(n)}$.

transmittance $\tilde{T}^{(n)}$ of 0.65 , and a minimum transmittance $\tilde{T}^{(n)}$ of 0.32 . To our knowledge this is the highest transmittance reported so far of a sample in the multiple scattering regime (thickness of 4 transport mean free paths). The transmittance enhancement $\eta_{T \text {,total }}^{(n)}$ for the thin sample is lower than we found for the thicker sample (Fig. 3.2). This is because even for $\tilde{T}^{(n)}=1$ we can only achieve an $\eta_{T \text {,total }}^{(n)}$ of $\tilde{T}_{\text {avg }}^{-1}=2.5$ with perfect control.

Using our high fidelity transmission matrix measurements, we achieve the highest reported single channel transmittance through an optically thick diffusive medium $[11-13,15]$. Our results demonstrate that the sum of the physical transmittance and reflectance within the collected aperture is constant. This shows that we do not only change the intensity of the transmitted light in an observed subset of the transmission aperture, but actually enhance the total transmittance.

\subsection{Calculating transmittance from an independent transmission matrix}

Experimentally, it is more convenient to work with measurements that allow offline data processing after the experiment is finished. In this section 
we consider such a measure for the transmission namely, the transmittance as predicted by an independent transmission matrix and demonstrate it increases with the transmittance through the sample, and gives a good measure for the true transmittance $\tilde{T}^{(n)}$.

The transmission eigenvalue $\Sigma^{(n)}$ is the transmittance of transmission channel $n$, calculated from the singular value of a measured transmission matrix, $\Sigma^{(n)}=\sigma^{(n) 2}$. The advantage of the transmission eigenvalue $\Sigma^{(n)}$ is that it is easy to calculate from a transmission matrix. The main disadvantages are that the presence of measurement noise in a transmission matrix can introduce a systematic error in the distribution of eigenvalues.

We calculate the transmittance by multiplying the transmission channel $v^{(n)}$ with the transmission matrix and calculating the modulus squared $T_{\Delta \lambda}^{(n)}=\left|T(\Delta \lambda) \boldsymbol{v}^{(n)}\right|^{2}$. We use an independently measured transmission matrix $T$ at any wavelength difference and specifically also at $\Delta \lambda=0$. This allows us to eliminate any systematic effects due to random measurement noise. The main advantage compared to the physical transmittance is that it requires only the measurement of the transmission matrices. This means that analysis can be performed in post processing, which allows high signal to noise measurements, and quick analysis. The main disadvantage is that we only measure the transmittance to the subspace of the modes over which we measure the transmission matrix. In this section we investigate how representative the transmittance $T_{\Delta \lambda}^{(n)}$ is to the physical transmittance $\tilde{T}^{(n)}$.

We plot the transmittance of the channels of a 5.2- $\mu \mathrm{m}$-thick sample in Fig. 3.4. The sample has an average transmittance $\tilde{T}_{\text {avg }}=0.26 \pm 0.03$, where we estimate the uncertainty in the measured transmittance from the undetected light due to the limited NA and field of view. In Fig. 3.4 (a) we plot the transmittance $T^{(n)}$ versus transmission eigenvalue $\Sigma^{(n)}$. We find a very small difference between the transmission and the transmission eigenvalue, which indicates a low amount of random noise in the transmission matrix measurements. In Fig. 3.4 (b) we plot the physical transmittance $\tilde{T}^{(n)}$ versus the transmittance $T^{(n)}$. We find a strong correlation between the physical transmittance $\tilde{T}^{(n)}$ and the transmittance $T^{(n)}$. This shows that $T^{(n)}$ is a good indicator for the physical transmittance. We find a transmittance $T^{(n)}$ ranging from 0 to 0.52 and $\tilde{T}^{(n)}$ ranging from 0.14 to 0.475 . 

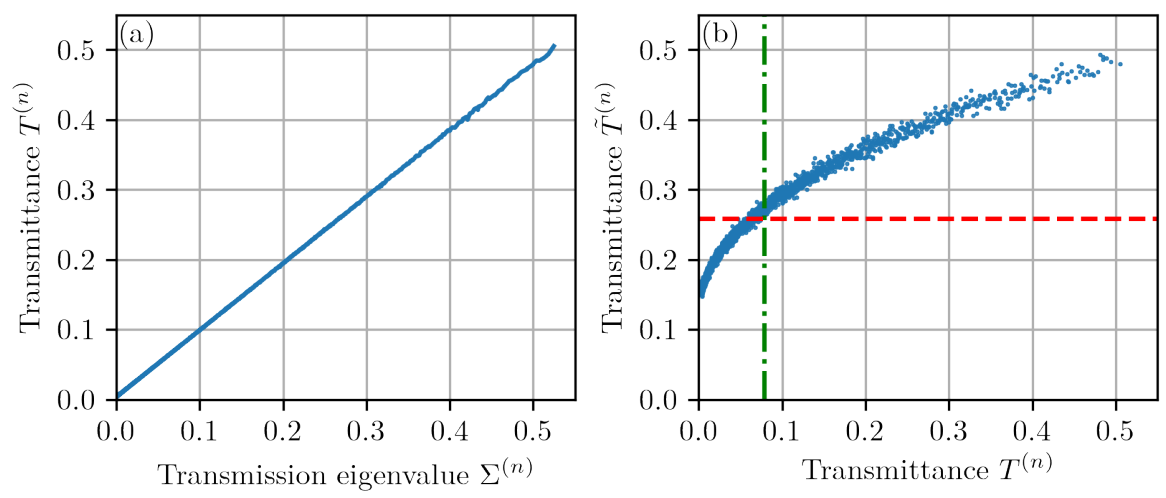

Figure 3.4.: Transmittance $T^{(n)}$ versus transmission eigenvalue $\Sigma^{(n)}$ (a). In (b) we plot the physical transmittance $\tilde{T}^{(n)}$ versus transmittance $T^{(n)}$. The data is for the 5.2- $\mu$ m-thick sample.

The difference between $T^{(n)}$ and $\tilde{T}^{(n)}$ can be explained by different contributions. The measurement of $T^{(n)}$ covers a limited numerical aperture and smaller area. From the measurements of $P_{r, T}^{(n)}$ and $P_{g, T}^{(n)}$ we find that $0.554 \pm 0.004$ of the captured transmitted power is within the sampled angular range. This, combined with the limited spatial sampling area, account for a large fraction of the difference. Finally due to the limited projection fidelity we do not perfectly project the transmission channels.

The transmittance $T^{(n)}$ calculated from the transmission matrix increases with the physical transmittance $\tilde{T}^{(n)}$. This demonstrates the transmittance $T^{(n)}$ is a good measure for the transmission of a transmission channel.

\subsection{Conclusions}

In conclusion, in this chapter we demonstrate measurements of the optical transmission matrix of a strongly scattering medium with a high fidelity and find and project the transmission channels of the medium. We project the transmission channels and increase and decrease the transmittance in a range from 0.32 to 0.65 , while simultaneously lowering and increasing the reflectance such that the total power is conserved. The directly measured transmittance of 0.65 is the highest reported so far for an optically thick 
scattering sample. The conservation of power over a large collection aperture in reflectance and transmission demonstrates we change the total transmitted power, and not just the power to a small angular subset of the transmitted modes.

Our results show that the transmission predicted by the transmission matrix increases with the physical transmittance, and is a good measure for transmittance through the sample. 


\section{Bibliography}

[1] C. W. J. Beenakker, "Random-matrix theory of quantum transport," Rev. Mod. Phys. 69, 731-808 (1997).

[2] M. C. W. van Rossum and T. M. Nieuwenhuizen, "Multiple scattering of classical waves : microscopy , mesoscopy , and diffusion," Rev. Mod. Phys. 71, 313-371 (1999).

[3] E. Akkermans and G. Montambaux, Mesoscopic Physics of Electrons and Photons (Cambridge University Press, 2007).

[4] S. Rotter and S. Gigan, "Light fields in complex media: Mesoscopic scattering meets wave control," Rev. Mod. Phys. 89, 015005 (2017).

[5] Y. Kuga and A. Ishimaru, "Retroreflectance from a dense distribution of spherical particles," J. Opt. Soc. Am. A 1, 831-835 (1984).

[6] M. P. V. Albada and A. Lagendijk, "Observation of weak localization of light in a random medium," Phys. Rev. Lett. 55, 2692-2695 (1985).

[7] P.-E. Wolf and G. Maret, "Weak localization and coherent backscattering of photons in disordered media," Phys. Rev. Lett. 55, 2696-2699 (1985).

[8] F. Scheffold and G. Maret, "Universal conductance fluctuations of light," Phys. Rev. Lett. 81, 5800-5803 (1998).

[9] I. M. Vellekoop and A. P. Mosk, "Focusing coherent light through opaque strongly scattering media," Opt. Lett. 32, 2309-2311 (2007).

[10] I. M. Vellekoop and A. P. Mosk, "Universal optimal transmission of light through disordered materials," Phys. Rev. Lett. 101, 120601 (2008).

[11] M. Kim, Y. Choi, C. Yoon, W. Choi, J. Kim, Q.-H. Park, and W. Choi, "Maximal energy transport through disordered media with the implementation of transmission eigenchannels," Nat. Photonics 6, 581-585 (2012).

[12] D. Kim, W. Choi, M. Kim, J. Moon, K. Seo, S. Ju, and W. Choi, "Implementing transmission eigenchannels of disordered media by a binarycontrol digital micromirror device," Opt. Commun. 330, 35 - 39 (2014).

[13] S. M. Popoff, A. Goetschy, S. Liew, A. Stone, and H. Cao, "Coherent control of total transmission of light through disordered media," Phys. Rev. Lett. 112, 133903 (2014).

[14] W. Choi, M. Kim, D. Kim, C. Yoon, C. Fang-Yen, Q.-H. Park, and W. Choi, "Preferential coupling of an incident wave to reflection eigen- 
channels of disordered media," Sci. Rep. 5, 11393 (2015).

[15] M. Kim, W. Choi, C. Yoon, G. H. Kim, S. hyun Kim, G.-R. Yi, Q.H. Park, and W. Choi, "Exploring anti-reflection modes in disordered media," Opt. Express 23, 12740-12749 (2015).

[16] J. Bosch, S. A. Goorden, and A. P. Mosk, "Frequency width of open channels in multiple scattering media," Opt. Express 24, 26472-26478 (2016).

[17] J. Aulbach, B. Gjonaj, P. M. Johnson, A. P. Mosk, and A. Lagendijk, "Control of light transmission through opaque scattering media in space and time," Phys. Rev. Lett. 106, 103901:1 -4 (2011).

[18] O. Katz, E. Small, Y. Bromberg, and Y. Silberberg, "Focusing and compression of ultrashort pulses through scattering media," Nat. Photonics 5, 372-377 (2011).

[19] D. J. McCabe, A. Tajalli, D. R. Austin, P. Bondareff, I. A. Walmsley, S. Gigan, and B. Chatel, "Spatio-temporal focusing of an ultrafast pulse through a multiply scattering medium," Nat. Commun. 2, 447 (2011).

[20] M. Mounaix, H. Defienne, and S. Gigan, "Deterministic light focusing in space and time through multiple scattering media with a time-resolved transmission matrix approach," Phys. Rev. A 94, 041802 (2016).

[21] M. Mounaix, D. Andreoli, H. Defienne, G. Volpe, O. Katz, S. Grésillon, and S. Gigan, "Spatiotemporal coherent control of light through a multiple scattering medium with the multispectral transmission matrix," Phys. Rev. Lett. 116, 253901 (2016).

[22] S. R. Huisman, T. J. Huisman, S. A. Goorden, A. P. Mosk, and P. W. H. Pinkse, "Programming balanced optical beam splitters in white paint," Opt. Express 22, 8320-8332 (2014).

[23] S. R. Huisman, T. J. Huisman, T. A. W. Wolterink, A. P. Mosk, and P. W. H. Pinkse, "Programmable multiport optical circuits in opaque scattering materials," Opt. Express 23, 3102-3116 (2015).

[24] T. A. W. Wolterink, R. Uppu, G. Ctistis, W. L. Vos, K.-J. Boller, and P. W. H. Pinkse, "Programmable two-photon quantum interference in $10^{3}$ channels in opaque scattering media," Phys. Rev. A 93, 053817 (2016).

[25] H. Defienne, M. Barbieri, B. Chalopin, B. Chatel, I. A. Walmsley, B. J. Smith, and S. Gigan, "Nonclassical light manipulation in a multiplescattering medium," Opt. Lett. 39, 6090-6093 (2014). 
[26] T. J. Huisman, S. R. Huisman, A. P. Mosk, and P. W. H. Pinkse, "Controlling single-photon fock-state propagation through opaque scattering media," Appl. Phys. B 116, 603-607 (2014).

[27] A. Aubry and A. Derode, "Random matrix theory applied to acoustic backscattering and imaging in complex media," Phys. Rev. Lett. 102, 084301 (2009).

[28] S. Popoff, G. Lerosey, R. Carminati, M. Fink, A. Boccara, and S. Gigan, "Measuring the transmission matrix in optics: An approach to the study and control of light propagation in disordered media," Phys. Rev. Lett. 104, 100601 (2010).

[29] S. M. Popoff, A. Aubry, G. Lerosey, M. Fink, A. C. Boccara, and S. Gigan, "Exploiting the time-reversal operator for adaptive optics, selective focusing, and scattering pattern analysis," Phys. Rev. Lett. 107, 263901 (2011).

[30] H. Yu, T. R. Hillman, W. Choi, J. O. Lee, M. S. Feld, R. R. Dasari, and Y. Park, "Measuring large optical transmission matrices of disordered media," Phys. Rev. Lett. 111, 153902 (2013).

[31] S. Popoff, G. Lerosey, M. Fink, A. C. Boccara, and S. Gigan, "Image transmission through an opaque material," Nat. Commun. 1, 81 (2010).

[32] P. Pai, J. Bosch, and A. P. Mosk, "Optical transmission matrix measurement sampled on a dense hexagonal lattice," OSA Continuum 3, 637-648 (2020).

[33] E. G. van Putten, "Disorder-enhanced imaging with spatially controlled light," PhD thesis, Universiteit Twente, Enschede (2011).

[34] P. Pai, "Accurate statistics from optical transmission matrix measurements," PhD thesis, Universiteit Utrecht (2020).

[35] V. A. Marčenko and L. A. Pastur, "Distribution of eigenvalues for some sets of random matrices," Math. USSR Sb. 1, 457-483 (1967).

[36] M. U. Vera and D. J. Durian, "Angular distribution of diffusely transmitted light," Phys. Rev. E 53, 3215-3224 (1996).

[37] H. Yu, K. Lee, and Y. Park, "Energy leakage in partially measured scattering matrices of disordered media," Phys. Rev. B 93, 104202 (2016). 



\section{Spectral width of transmission channels ${ }^{1}$}

\subsection{Introduction}

Mesoscopic transport theory successfully explains correlations in the transmission of light [1-3]. Transmission channels are a key element of this theory [4-8] and have been observed in experiments [9-14]. However the properties of the individual transmission channels are theoretically [and experimentally] not well explored. For the practical application of transmission channels, their bandwidth is an important property $[8,15]$. Moreover the bandwidth of a transmission channel can give an insight to its relation to resonances in the medium [16]. The crucial question of how the transmittance of a transmission channel changes as a function of the wavelength has as of yet only been partially explored.

The bandwidth of individual transmission channels in a microwave waveguide with a limited number of modes has been measured [14,17], it is an open question whether these results are relevant for the slab type samples that are typical in the optical regime. There is also experimental work in the optical diffusive regime [18], however this measurement only addresses one transmission channel with a limited increase in transmittance. Numerical work has been performed both in the localized [19] and the diffuse regime [20], however these simulations use a 2D waveguide geometry. Here we experimentally investigate the transmission channels of a $3 \mathrm{D}$ diffusive strongly scattering medium. We identify transmission channels with a very transmittance of more than six times the average transmittance as well as the

\footnotetext{
${ }^{1}$ This chapter is part of a collaborative work with Hui Chao, Sanli Faez, Chia Wei Hsu, Allard P. Mosk, Pritam Pai, Alexey Yamilov, Hasan Yilmax. The experimental work presented here has been performed by Jeroen Bosch
} 
transmission channels with average and near zero transmittance. We investigate the transmittance as a function of wavelength detuning of all these groups of transmission channels. We find a universal line shape for the transmission channels with above average transmittance and a highly anomalous line shape for the transmission channels with average transmittance. The fidelity of the measurement is shown to be of critical importance of the lineshape. Finally we investigate how the transmitted field changes shape when changing the wavelength of the incident light.

\subsection{Theory}

Mesoscopic transport theory describes frequency-dependent phenomena through correlation functions. The speckle correlation function is typically given as $[21,22]$

$$
C(\Delta \omega)=\left\langle T_{a b}(\omega) T_{c d}(\omega+\Delta \omega)\right\rangle /\left(\left\langle T_{a b}(\omega)\right\rangle\left\langle T_{c d}(\omega+\Delta \omega)\right\rangle\right),
$$

where \langle\rangle indicates ensemble averaging. Typically the $C(\Delta \omega)$ correlation function is measured as an intensity correlation, as this is more robust than measuring field correlations in most experiments. Our experiment we measure field correlations because we reliably measure the field. Berkovits et al. [23] have shown that it is possible to calculate the speckle correlation $C$ from the field correlations,

$$
C(\Delta \omega)=\left|\left\langle E_{\boldsymbol{k}}(\omega)^{*} E_{\boldsymbol{k}}(\omega+\Delta \omega)\right\rangle\right|^{2},
$$

which is an average over the ensemble of scattering samples and normalized such that $C(\Delta \omega=0)=1$. The speckle correlation function has three different contributions: $C=C^{(1)}+C^{(2)}+C^{(3)}$. The leading contributions of the correlation functions are of order $1,1 / g$ and $1 / g^{2}$. In the weak-disorder regime in which we investigate $g \approx N l / L$ with $l$ the mean free path and $N$ the number of channels. The $C^{(1)}$ correlation function has been measured experimentally for angular behavior [24] and for spectral behavior [25]. The $C^{(2)}$ and $C^{(3)}$ have been measured in the time domain [26]

Van Rossum and Nieuwhuizen give an algebraic solution for the $C^{(1)}$ correlation [22]:

$$
C^{(1)}(\Delta \omega)=\frac{|M|^{2}}{\kappa^{2}}\left|\frac{\left(1+\kappa^{2} z_{0}^{2}\right) \sinh \kappa L+2 \kappa z_{0} \cosh \kappa L}{\left(1+M^{2} z_{0}^{2}\right) \sinh M L+2 M z_{0} \cosh M L}\right|^{2},
$$


where, $M^{2}=q_{\perp}^{2}+\kappa^{2}+i \frac{\Delta \omega}{D}, \kappa=L_{a}^{-1}$ denotes the inverse absorption length, $q_{\perp}^{2}$ the perpendicular momentum difference, $z_{0}$ the extrapolation length and $L$ the sample thickness. We use the approximation with no absorption for $C^{(2)}$ and neglect boundary reflections, because $L>z_{0}$ and $L<<L_{a}$. In its normalized form $C^{(2)}$ is given by de Boer [22,27]

$$
C^{(2)}(\tilde{\Omega})=\frac{2}{\sqrt{2 \tilde{\Omega}} L}\left(\frac{\sinh (\sqrt{2 \tilde{\Omega}} L)-\sin (\sqrt{2 \tilde{\Omega}} L)}{\cosh (\sqrt{2 \tilde{\Omega}} L)-\cos (\sqrt{2 \tilde{\Omega}} L)}\right),
$$

where $\tilde{\Omega}$ denotes the reduced frequency $\tilde{\Omega}=\Delta \omega / D$. We use the normalized form of $C^{(2)}$, as we are interested in the spectral shape of the correlation function. We do not consider the $C^{(3)}$ as they are negligibly small due to the typical value of $g \approx 60$.

The characteristic bandwidth associated with a multiply scattering material is the Thouless width. We estimate the Thouless bandwidth $\omega_{\text {Th }}$ consistently as $C\left(\omega_{\mathrm{Th}}\right)^{(1)}=0.5$. Neglecting boundary effects, the Thouless bandwidth scales as $\omega_{\mathrm{Th}} \propto \frac{D}{L^{2}}$. The Thouless width is much larger than the frequency of the light $\omega_{\mathrm{Th}} \ll \omega_{0}$ which allows us to define the Thouless width in units of the wavelength. We measure the Thouless width $\Delta \lambda_{\mathrm{Th}}$, as $C\left(\Delta \lambda_{\mathrm{Th}}\right)^{(1)}=0.5$.

We investigate the transmittance of individual transmission channels, for which there is no known theory. However, we hypothesize that we can describe the line shape of the transmittance as a function of detuning and the correlation function of the transmitted transmission channels with a weighted sum of the $C^{(1)}$ and $C^{(2)}$ correlation functions.

\subsection{Correlation function from a multi spectral transmission matrix measurement}

In this section we briefly describe the parameter choices within the transmission matrix procedure and we find the speckle correlation function of the measured sample based on the MSTM.

We measure the MSTM based on the measurement procedure as described in Chapter 2 at 6 different wavelengths. We correct for dispersion in the experiment by adjusting the hologram, creating one set of corrected holograms per wavelength. The size of the frame buffer limits measurements to 
six wavelengths. We choose a wavelength stepsize of $1 \mathrm{~nm}$. At this stepsize, adjacent transmission matrices of a typical 5.2- $\mu$ m-thick sample have a high correlation of 0.68 . We measure the transmission matrices starting at $771 \mathrm{~nm}$. For every wavelength two transmission matrices are measured in a sawtooth-wave manner, for a total of twelve transmission matrices. This ensures two independently measured transmission matrices at every wavelength with equal time delay between their measurement. Data collection takes about 22 minutes per transmission matrix.

In Fig. 4.1 we plot the full correlations between the transmission matrices of the MSTM, calculated according to

$$
C\left(\Delta \lambda_{1}, \Delta \lambda_{2}\right)=\frac{\left|\sum_{i, j} T_{i, j}^{\dagger}\left(\lambda_{0}+\Delta \lambda_{1}\right) T_{i, j}\left(\lambda_{0}+\Delta \lambda_{2}\right)\right|^{2}}{\sum_{i, j}\left|T_{i, j}\left(\lambda_{0}+\Delta \lambda_{1}\right)\right|^{2} \sum_{i, j}\left|T_{i, j}\left(\lambda_{0}+\Delta \lambda_{2}\right)\right|^{2}},
$$

where $T_{i, j}\left(\lambda_{0}+\Delta \lambda_{1}\right)$ is a transmission matrix element measured at wavelength $\lambda_{0}+\Delta \lambda_{1}$. The sample is $5.2 \mu \mathrm{m}$ thick. We find a high correlation of 0.92 between transmission matrices (independently) measured at the same wavelength.

In Fig. 4.2 we plot the same data versus $\Delta \lambda$, and a fit of the $C^{(1)}$ function (Eq. 4.3). For the zero detuning datapoint we calculate the correlation on independently measured transmission matrices. We fit Eq. 4.3, with fixed parameters $z_{0}=1.15 \mu \mathrm{m}, q_{\perp}^{2}=0, \kappa^{2}=1 \mathrm{~m}, L=5.2 \mu \mathrm{m}$, and with only a single prefactor $a$ and the diffusion coefficient $D$ as free parameters. We find a diffusion constant $D=30 \mathrm{~m}^{2} / \mathrm{s}$, which is in line with samples prepared according to the same procedure from similar materials [28]. We estimate a value $g \approx 60$, so that a $C^{(2)}$ contribution is not observable here. 


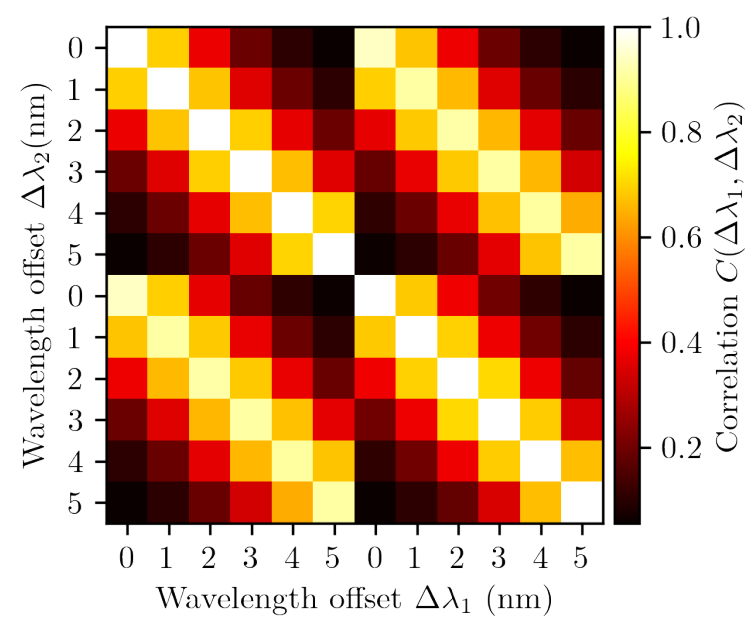

Figure 4.1.: Correlation coefficient between transmission matrix with different wavelength offsets. The matrices are ordered along the horizontal and vertical axis according to order of measurement. Transmission matrix at $0 \mathrm{~nm}$ offset was measured at $771 \mathrm{~nm}$. For independent matrices measured at the same wavelength we find a high correlation $C$ of 0.92 .

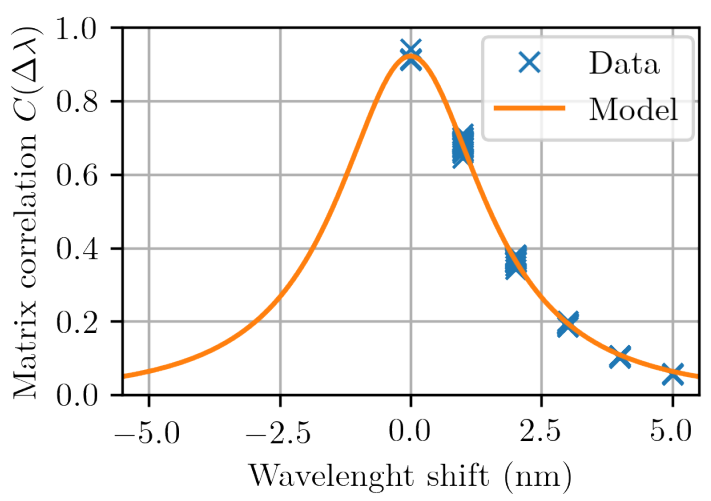

Figure 4.2.: Correlation coefficient between transmission matrices with different wavelength offsets (blue). In orange fit according to the $C^{(1)}$ correlation function, with $D=30 \mathrm{~m}^{2} / \mathrm{s}$. 


\subsection{Wavelength dependence of transmission enhancement of channels}

In this section we investigate the transmittance of transmission channels versus detuning. As a surprising result we find that within our measurement fidelity the spectral width of open channels is constant relative to the Thouless width, independent of transmittance of the channel, or sample thickness.

We calculate the detuned transmittance $T_{\Delta \lambda}^{(n)}$ of a transmission channel by multiplying the transmission matrix $T(\Delta \lambda)$ with the transmission channel to calculate the propagated field and calculating its vector norm squared: $T_{\Delta \lambda}^{(n)}=\left|T(\Delta \lambda) \boldsymbol{v}^{(n)}\right|^{2}$. We calculate the transmittance for all pairs of wavelengths, which leads to a different number of datapoints per detuning value, e.g. there are many more pairs spaced at $1 \mathrm{~nm}$, than at $5 \mathrm{~nm}$. We calculate the transmittance at a detuning of $0 \mathrm{~nm}$ from an independently measured transmission matrix i.e. the eigenvectors $\boldsymbol{v}^{(n)}$ and the transmittance $T_{\Delta \lambda}^{(n)}$ are calculated from different measurements to avoid spurious correlations due to noise (Chapter 3). In Fig. 4.3 we plot the detuned transmittance for different values of the detuning versus transmission eigenvalue $\Sigma^{(n)}$. We fit independent linear functions to the transmittance at every detuning, where we only take into account channels with a transmission eigenvalue higher than 0.05, as the channels with lower transmittance deviate from the linear behavior. The linear fits agree well with the results.

We identify three distinct regimes for the transmission channels from Fig. 4.3. The first regime is that of the transmission channels with a transmittance above the sample average transmittance where we can fit a linear relationship, and which are described in this section. The second regime is that of the transmission channels that have a transmittance of approximately the average transmittance. We describe these channels in Section 4.5. Finally the third regime is that of the transmission channels with a very low transmittance, where we see that the transmittance curve bends towards zero transmittance, indicating a very large spectral width for the suppressed transmittance.

We observe that the transmittance of a channel regresses towards the mean transmittance at large detuning, however it does not yet reach the mean transmittance at $5 \mathrm{~nm}$ detuning. The transmittance of the channels 


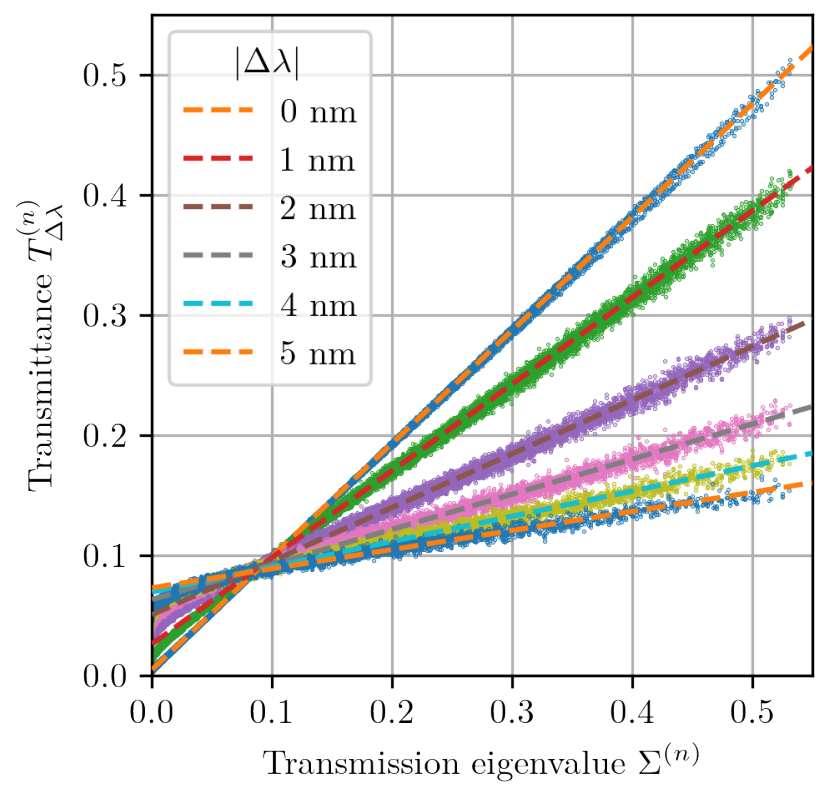

Figure 4.3.: Detuned transmittance of transmission channels versus transmission eigenvalue for different values of the detuning $|\Delta \lambda|$. Dotted lines are linear fits through the datapoints for transmission eigenvalues above 0.05 . The curves cross at a transmittance of 0.09. The legend indicates the wavelength detuning $|\Delta \lambda|$ corresponding to the line.

with above sample average transmittance can be described well by a linear regression. This means that we can associate a single spectral width to the transmission increase of the open transmission channels, and the spectral width of the increase in transmittance is not dependent on the transmittance.

In Fig. 4.4 we plot transmittance increase as a function for detuning for a channel with a transmittance of 0.59 . The transmittance increase is calculated from the linear fit to the transmittance versus channel transmittance (e.g. as in Fig. 4.3). There is no theoretical model describing the transmission increase as a function of detuning that we know of. However, we find that a linear combination of the correlation functions $a\left(C^{(1)}(\Delta \lambda)+b C^{(2)}(\Delta \lambda)\right)$ 


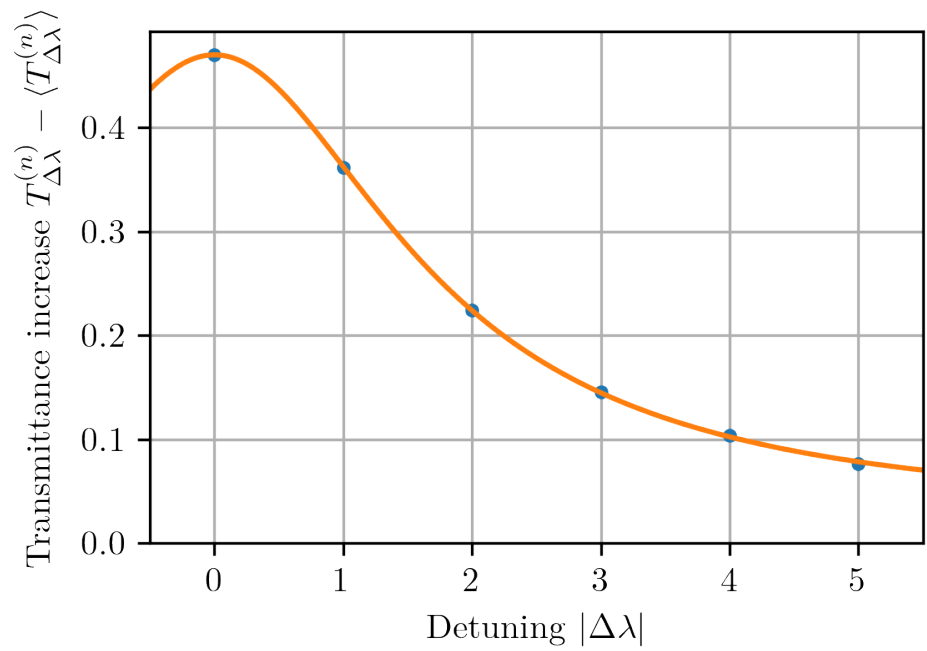

Figure 4.4.: Transmittance increase as a function of detuning for a transmission channel with a transmittance of 0.59 . The datapoints are derived from the linear fits, the line indicates a fit according to $a\left(C^{(1)}(\Delta \lambda)+b C^{(2)}(\Delta \lambda)\right)$.

(Eq. (4.3) and Eq. (4.4)), gives a good fit with the measurement result (Fig. 4.4). We use 3 free parameters: $a, b$ and the diffusion coefficient $D$. We find a value of $b$ of 0.39 . The spectral width of the transmittance enhancement, defined as the full width at half maximum of the fit, is $15 \%$ wider than the Thouless width. This is less than the $43 \%$ to $93 \%$ increase in width previously reported [18] from a single polarization optimization through iterative phase conjugation. The present work is based on a different measurement method and on a larger dataset of hundreds of channels that optimizes for both polarization components.

We now examine the spectral width of the transmission enhancement in samples of different thickness. For this we perform the measurement on five different sample thicknesses (Table 4.1). We perform a physical measurement of the sample thickness by focusing on the sample surface from both sides (Chapter 2), with an estimated systematic error of up to $1 \mu \mathrm{m}$. This systematic error leads to a significant spread in the calculated diffusion co- 


\begin{tabular}{|l|l|l|l|l|l|}
\hline Physical thickness $(\mu \mathrm{m})$ & 2.7 & 5.2 & 10.8 & 8.9 & 15.6 \\
\hline Thouless width $(\mathrm{nm})$ & 2.42 & 1.64 & 1.26 & 1.03 & 0.68 \\
\hline Converted thickness $(\mu \mathrm{m})$ & 3.9 & 5.2 & 6.15 & 7.0 & 9.15 \\
\hline
\end{tabular}

Table 4.1.: List of samples and properties.

efficients. We therefore calculate an effective sample thickness assuming a diffusion constant of 30 from the Thouless width. The discrepancy between the physical thickness and the calculated effective thickness can be due to errors in the thickness measurement or to a position dependent diffusion constant. This can be caused by a thickness dependence in the physics of the drying and a variation in the consistency of the $\mathrm{ZnO}$ particles.

To compare the spectral width of the transmittance increase or decrease we combine the transmission data at different thickness. For this we plot the transmittance increase versus detuning in units of the Thouless width as extracted from the whole-matrix correlation. In Fig. 4.5 we show the transmittance increase as a function of detuning (colored dots) for different sample thickness. Again, we fit a combination of $a\left(C^{(1)}(\Delta \lambda)+b C^{(2)}(\Delta \lambda)\right)$. From this data it is clear that the spectral width of the increase in transmission shows a universal behavior independent of the effective sample thickness. We find a half width at half maximum (HWHM) spectral width of transmission enhancement of 1.18 times the Thouless width, and a $C^{(2)}(\Delta \lambda)$ mixing value $b$ of 0.34 , which is remarkably close to our estimated fidelity $F \approx 0.36$. This is in contrast to the full-matrix correlation function, which has only a negligibly small $C^{(2)}(\Delta \lambda)$ contribution which is not resolvable here due to experimental uncertainties. These results seem to indicate that addressing single transmission channels manifests $C^{(2)}$ contribution, as was independently observed in the transmission intensity statistics [29]. 


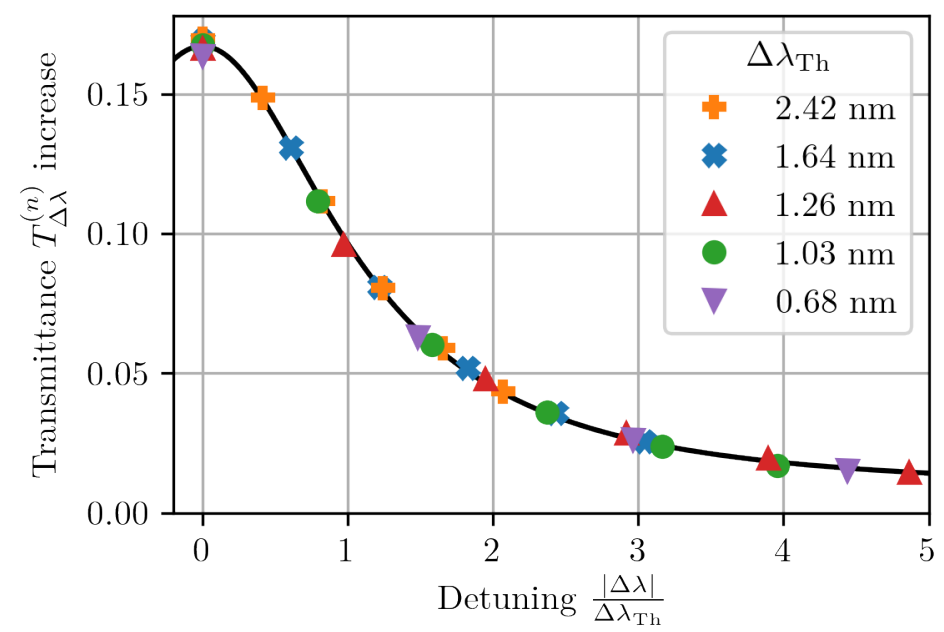

Figure 4.5.: Transmittance increase as a function of detuning for samples with different thickness. The samples are labeled according to their Thouless width and labeled with different symbols. In black the model $a\left(C^{(1)}+b C^{(2)}\right)$.

\subsection{Anomalous transmittance of transmission channels}

In this section, we take a close look at the transmission channels with a transmittance that is slightly above to below the average transmittance. We investigate how the transmittance of these transmission channels changes if we change the wavelength of the light.

In Fig. 4.3 the crossing of the transmittance versus the transmission channels at different detuning is not well resolved due to noise. Within the noise, it seems as if all lines cross. This implies that the transmittance of a channel with average transmittance is independent of detuning. In order to investigate whether the transmittance of a channel with average transmittance is actually independent of detuning, we need to reduce the noise. We reduce the noise by binning the transmission channels according to their transmission eigenvalue, and calculating the average transmittance for every detuning. 


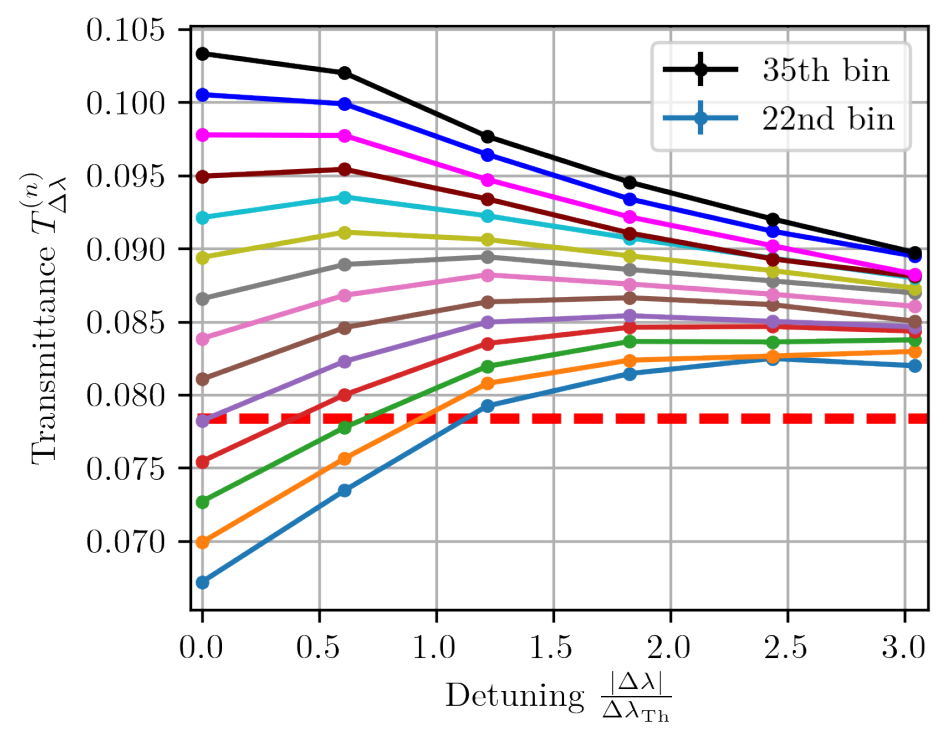

Figure 4.6.: Transmittance versus detuning for the transmission channels with a transmission eigenvalue in the 22nd $\left(T_{\Delta \lambda}=0.067\right)$ to 35 th $\left(T_{\Delta \lambda}=0.103\right)$ bin out of a total of 180 bins. The average transmittance of the 5.2- $\mu \mathrm{m}$-thick sample is indicated with the thick red dotted line. The datapoints are connected as a guide to the eye. The errorbars (standard deviation of the mean) are smaller than the symbol size.

We use 180 bins of equal width, and calculate the data for the transmission channels of a 5.2-um-thick sample.

In Fig. 4.6 we plot the transmittance versus detuning for the transmission channels with a transmission eigenvalue in the $22 \mathrm{nd}\left(T_{\Delta \lambda=0}=0.067\right)$ to 35 th $\left(T_{\Delta \lambda=0}=0.103\right)$ bin out of the total of 180 bins. There are between 53 and 409 channels per bin. We also plot the average transmittance $\left(T_{\mathrm{avg}}=0.078\right)$ of the 5.2-um-thick sample.

Surprisingly, the transmission channels with the average transmittance at zero detuning have an above average transmittance when propagated at a detuned wavelength. Even for transmission channels with below average transmittance, we find that the transmittance increases to above average 
transmittance at a detuning of roughly twice the Thouless width. These results are surprising as one would intuitively expect that by detuning the transmission regresses monotonically towards the average transmittance, because the transmission matrix decorrelates with respect to the transmission channel, which would also be consistent with earlier measurements [18, 30]. We find this anomalous non-monotonic transmittance at all five measured sample thicknesses.

\subsection{Influence of fidelity on transmission enhancement}

In this section, we investigate the role of the transmission matrix fidelity on the measured spectral width and spectral shape of the increase in transmission of transmission channels.

The transmission matrix $T$ is a representation of the transmission operator of the sample. In an experiment we do not sample the full transmission operator of the material since we sample only a part of the incident and transmitted modes. Additionally there are measurement errors which reduce the fidelity. We have demonstrated in section 2.2 that we can approximate the fidelity $F$ as $F_{\mathrm{TM}}=\frac{\min (m, n)}{N}$ (Eq. (2.4), where $N$ is the number of optical modes of the sample, and $n$ and $m$ the number of incident and transmitted sampled modes. Here we investigate the influence of the fidelity of the transmission matrix by reducing the number of sampled incident and transmitted modes by randomly removing columns and rows of the transmission matrix. To decrease control we pick a number of modes $n$, and reduce the matrix size from $2282 \times 2282$ to $n \times n$, by selecting $n$ random rows and $n$ random columns of the full measured transmission matrix [31]. We rescale the amplitude of the individual matrix elements with $\sqrt{\frac{2282}{n}}$ to keep the average transmittance $\tilde{T}_{\text {avg }}$ constant. For a transmission matrix of $2282 \times 2282$ we estimate a fidelity of 0.36 .

In Fig. 4.7 we plot the transmittance $T_{\Delta \lambda}^{(n)}$ versus eigenvalue for different amounts of detuning calculated for different transmission matrix fidelity. In (a) we plot the results for a $n=700(F \approx 0.11)$ subspace of the transmission matrix and in (b) for the full measured transmission matrix $(F \approx 0.36)$. We find that the transmittance behavior of the highly transmitting transmission 
channels is linear as a function of transmission eigenvalue for both degrees of control. However for the transmission channels with a transmittance below the average transmittance we see a deviation for the high fidelity result. In (c) and (d) we zoom-in on the low transmittance channels of (a) and (b) respectively. In order to resolve the transmittance we bin the transmission eigenvalue and plot the average transmittance value in every bin. We find that for the low fidelity (c) the transmittance curves cross in a single point, that is approximately equal to the average matrix transmittance (0.078). For the full transmission matrix we find that the transmittance curves show anomalous transmittance, and the approximate crossing is at a slightly higher transmittance (0.09). Additionally we see that for the full transmission matrix the low transmittance channels have an increased spectral bandwidth, as is indicated by the bending below transmission eigenvalues $\Sigma^{(n)}$ of 0.01 . The lack of these features for a transmission matrix with a low degree of control indicates it is an effect of the physics of mesoscopic light transport that is only resolved at a high fidelity.

For lower fidelity data we also calculate the spectral lineshape of the transmittance increase. We find that the shape of the curve depends on the degree of control. In Fig. 4.8 we plot the transmittance versus detuning for a channel with low control (a, $N=700, F \approx 0.11$ ) and for the full transmission matrix (b, $F \approx 0.36$ ), we include the datapoints of every sample thickness. We fit $a\left(C^{(1)}+b C^{(2)}\right)$ (Eq. (4.3) and (4.4)) to the data. We find that for a low fidelity $C^{(1)}$ fits well to the data, and for best fidelity we need a significant contribution of $C^{(2)}$.

We calculate the shape of the transmittance increase versus detuning for different matrix sizes. For every matrix size we fit a combination of $a\left(C^{(1)}+\right.$ $\left.b C^{(2)}\right)$ with three fit parameters $a, b$ and $D$ to the datapoints, we include the datapoints of every sample thickness. We find the model fits well to the data points. In Fig. 4.9 (b) we plot the fit parameter $b$ versus matrix size and we plot the estimated transmission matrix fidelity. We find that the transmission matrix fidelity is proportional to $b$ to good approximation. In Fig. 4.9 (a) we plot the full width at half maximum of the fit. We find that the width of the transmittance increase linearly increases with the fidelity of the transmission matrix.

These results demonstrate that the measured spectral shape of the transmittance increase of transmission channels is dependent on the fidelity of 
the measurement. It shows that in order to resolve the long range $C^{(2)}$ correlations, the transmission matrix has to be sampled with a high fidelity. 

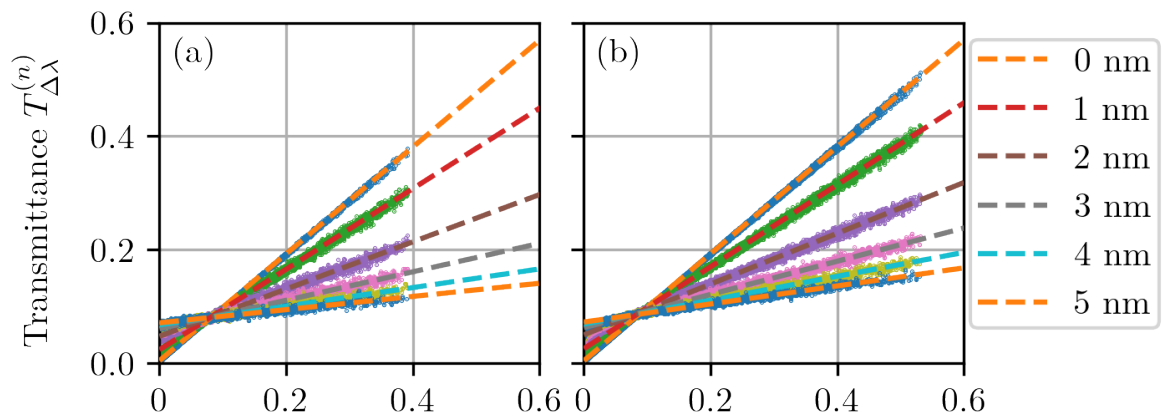

Transmittance eigenvalue $\Sigma^{(n)}$ Transmittance eigenvalue $\Sigma^{(n)}$
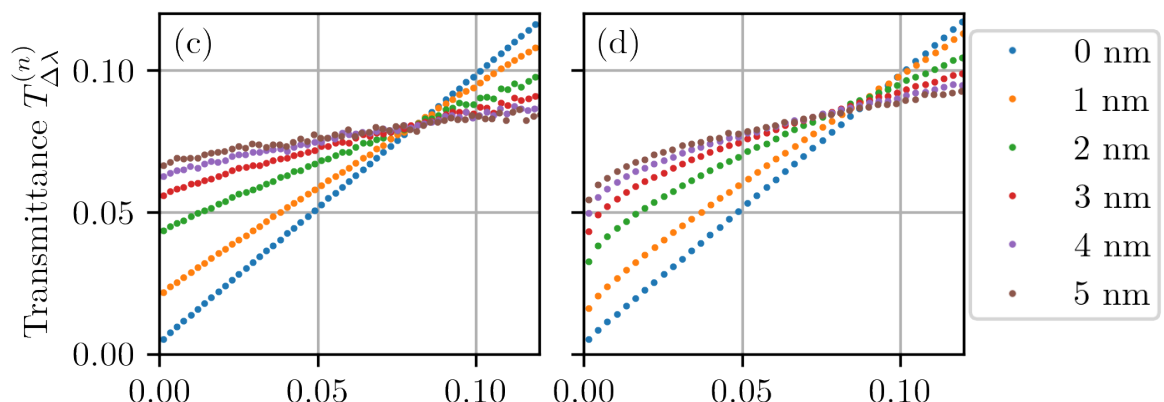

0.00

$0.10 \quad 0.00$

0.05

0.10

Transmittance eigenvalue $\Sigma^{(n)}$ Transmittance eigenvalue $\Sigma^{(n)}$

Figure 4.7.: Transmittance $T_{\Delta \lambda}^{(n)}$ versus transmission eigenvalue $\Sigma^{(n)}$ for two degrees of control. In (a,c) for a $N=700$ subspace of the transmission matrix, in $(b, d)$ for the full measured transmission matrix. The colors indicate the detuning $\Delta \lambda$. In (c) and (d) we plot a a zoom-in on (a) and (b) respectively, where the transmission eigenvalues are binned and we plot the average transmittance per bin. The data is from the 5.2- $\mu \mathrm{m}$-thick sample. 


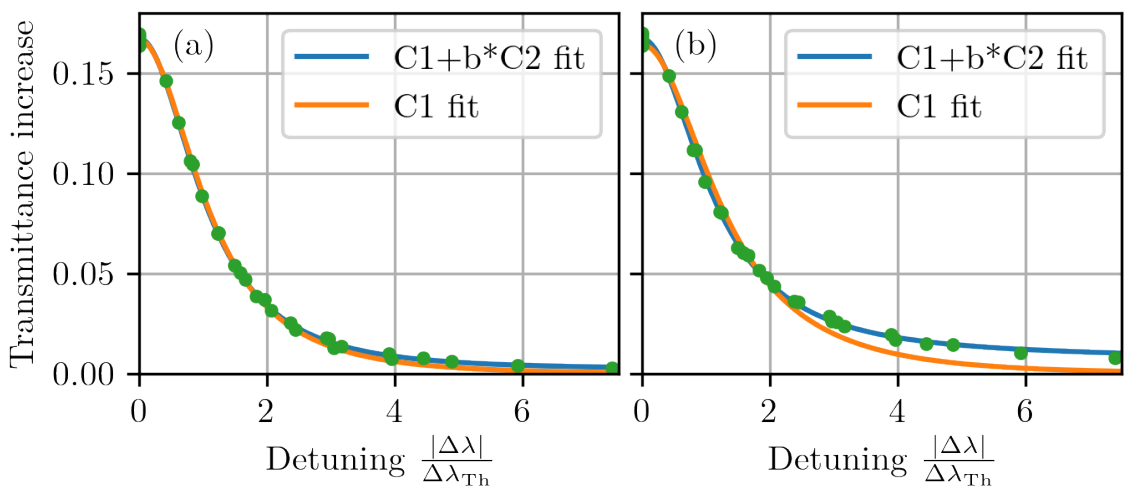

Figure 4.8.: Spectral line shape of the transmittance increase for two transmission matrices with a different fidelity and two model fits. In (a) a fidelity of $F \approx 0.11$ and (b) a fidelity of $F \approx 0.36$.
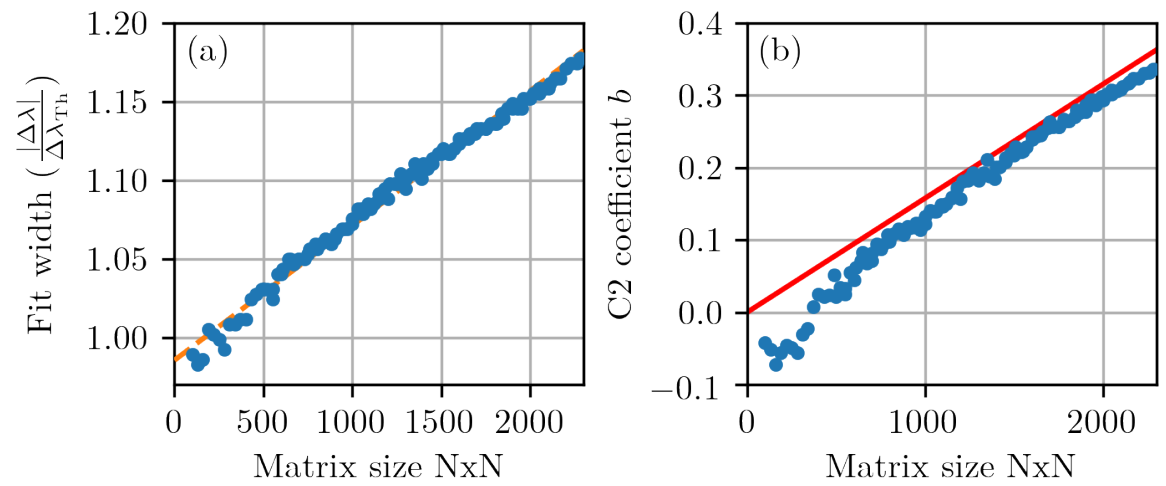

Figure 4.9.: Spectral width of the transmittance increase versus detuning for different transmission matrix sizes. In (a) the half width at half maximum calculated from a $a\left(C^{(1)}(\Delta \lambda)+b C^{(2)}(\Delta \lambda)\right)$ model fit. Data points in blue, in orange a linear regression to the data points. In (b) we plot the fit parameter $b$ versus matrix size (dots) and the estimated transmission matrix fidelity $F$ (line). 


\subsection{Correlation width of transmission channels}

In this section, we investigate the correlation width of transmission channels. We investigate how the transmitted field profile of a transmission channel changes if we change the wavelength of the light. For this we calculate the transmission channels at a wavelength $\lambda_{0}$, and propagate the fields at five different wavelength for which we have measured a transmission matrix. We define the cosine similarity, which calculates the similarity between two vectors:

$$
C S(\boldsymbol{a}, \boldsymbol{b})=\frac{|\boldsymbol{a} \cdot \boldsymbol{b}|}{|\boldsymbol{a}||\boldsymbol{b}|},
$$

where $|\boldsymbol{a} \cdot \boldsymbol{b}|$ denotes the norm of the inner product between $\boldsymbol{a}$ and $\boldsymbol{b}$, and $|\boldsymbol{a}|$ the norm of $\boldsymbol{a}$. We calculate the propagated field by multiplication of transmission channel $\boldsymbol{v}^{(n)}$ with the transmission matrix at a different wavelength $T\left(\lambda_{0}\right) \boldsymbol{v}^{(n)}$. In order to compare the transmission channels with the speckle correlation function, we square the cosine similarity:

$$
C_{W, \lambda_{0}}^{(n)}(\Delta \lambda)=C S\left(T\left(\lambda_{0}\right) \boldsymbol{v}^{(n)}, T(\Delta \lambda) \boldsymbol{v}^{(n)}\right)^{2}
$$

where $\boldsymbol{v}_{n}$ denotes the input field of the $n$-th transmission channel, calculated at $\lambda_{0}$. We do not perform an ensemble averaging over different transmission channels. For $\Delta \lambda=0$ we propagate the vector through a transmission matrix that is measured independently from the one that was used to calculate the channels.

The $C_{W, \lambda_{0}=771 \mathrm{~nm}}^{(n)}(\Delta \lambda)$ correlations calculated according to Equation 4.7 are plotted in Fig. 4.10. We find that the transmission channels propagated at $\Delta \lambda=0$ have a very high correlation $>0.8$ for all transmission channels with a transmittance above 0.016. Moreover we find the same correlation coefficient between transmission matrices at the same $\Delta \lambda$. This is a good indicator of the precision and repeatability of our transmission matrix measurements. The low correlations for the very low transmission channels can be explained by the fact that these transmission channels are more sensitive to measurement noise, as they have a much lower transmitted intensity.

We fit Equation 4.3 to the correlation function of every channel, and calculate the spectral width. In Fig. 4.11 we plot the correlation values $C_{W, \lambda_{0}=771 \mathrm{~nm}}^{(n)}(\Delta \lambda)$, a fit of the data points to a $C^{(1)}$ function with amplitude $a$ and diffusion constant $D$ as fit parameters for a transmission channel with 


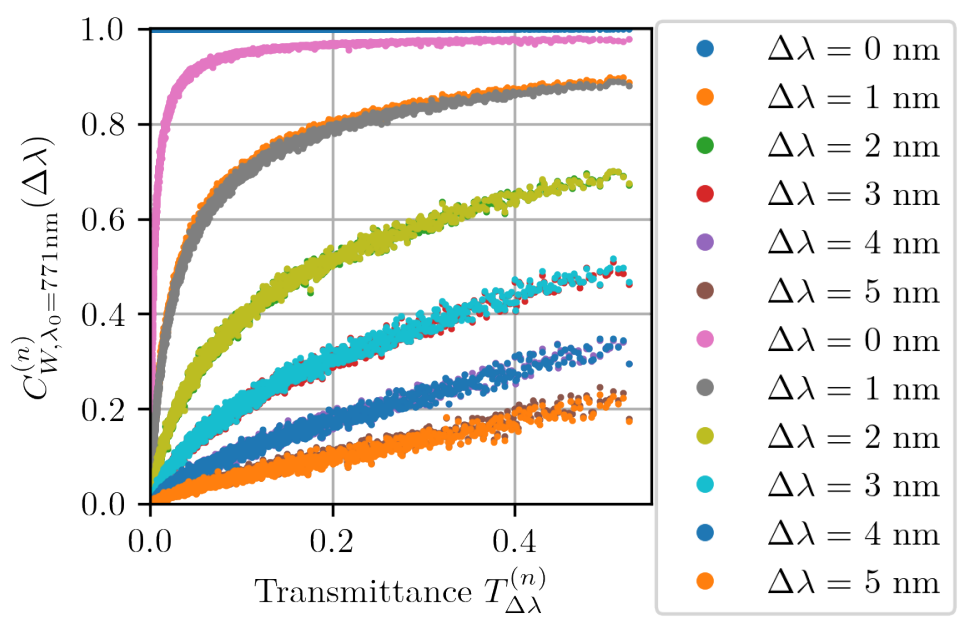

Figure 4.10.: Correlation function of transmission channels propagated at different wavelengths through the transmission matrix. The transmission channels are calculated at $\lambda_{0}=771 \mathrm{~nm}$, propagated at the indicated wavelengths, and we calculate the correlation from the two propagated fields. We find a good correspondence for the correlations between the independently measured transmission matrices.

$\Sigma^{(n)}=0.52$, and the $C^{(1)}$ correlation function of all elements of the transmission matrix (green). We find that the model fits well to the data, and we find a $82 \%$ increase in width compared to the Thouless width. Remarkably we do not need a $C^{(2)}$ contribution to describe the correlation function.

We plot the width of the $C_{W, \lambda_{0}}(\Delta \lambda)$ correlation function versus transmittance in Fig. 4.12 (a). We find an increase in spectral width of more than $75 \%$ for the highest transmission eigenvalue, which is more than 6 times the average. In earlier reported results a spectral width of $14 \%$ to $24 \%$ was reported [18] for a channel with a $54 \%$ increase in transmittance. We find a similar increase in spectral width for the same increase in transmittance.

We also investigate the thickness dependence of the spectral correlation width. For this we calculate $C_{W}^{(n)}$ for samples with different thickness. We use the same datasets as for the wavelength dependence of the transmission enhancement. For every sample thickness we calculate the correlation width 


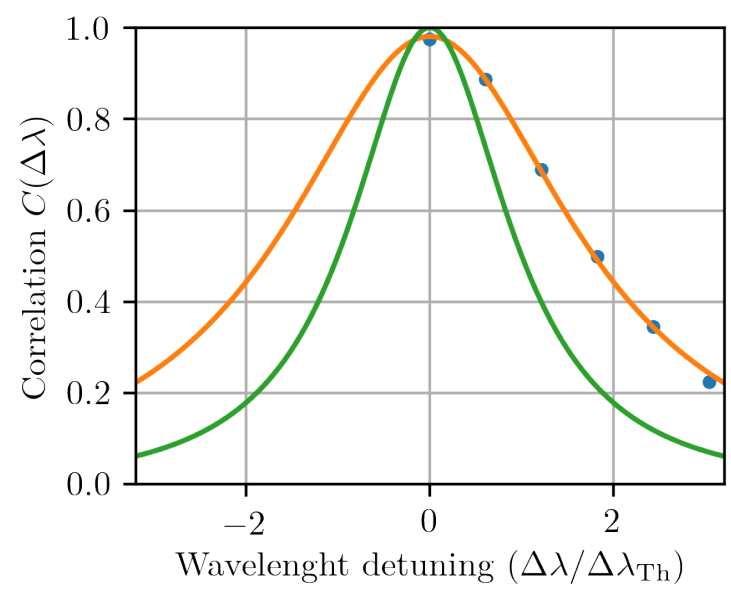

Figure 4.11.: Field correlation $C_{W, \lambda_{0}=771 \mathrm{~nm}}^{(n)}(\Delta \lambda)$ versus detuning (blue) with $C^{(1)}$ fit (orange). The $C^{(1)}$ correlation function of the corresponding sample is plotted in green.

as a function as transmittance, in the same way as for Fig. 4.12 (a). We bin the transmission eigenvalues over 90 equally spaced bins, and calculate the average spectral width and standard deviation of the spectral width for every bin, to find a single curve of $C_{W}^{(n)}$ as a function of transmittance per sample thickness.

In Fig. 4.12(b) we plot the spectral width versus the transmission channel eigenvalue $\Sigma^{(n)} / \Sigma_{\max }^{(n)}$, where $\Sigma_{\max }^{(n)}$ is the maximum transmission eigenvalue of the respective sample thickness. We find that the relative spectral width of the transmission channel is independent of the sample thickness, and independent of the maximum transmittance of the transmission channels observed. 

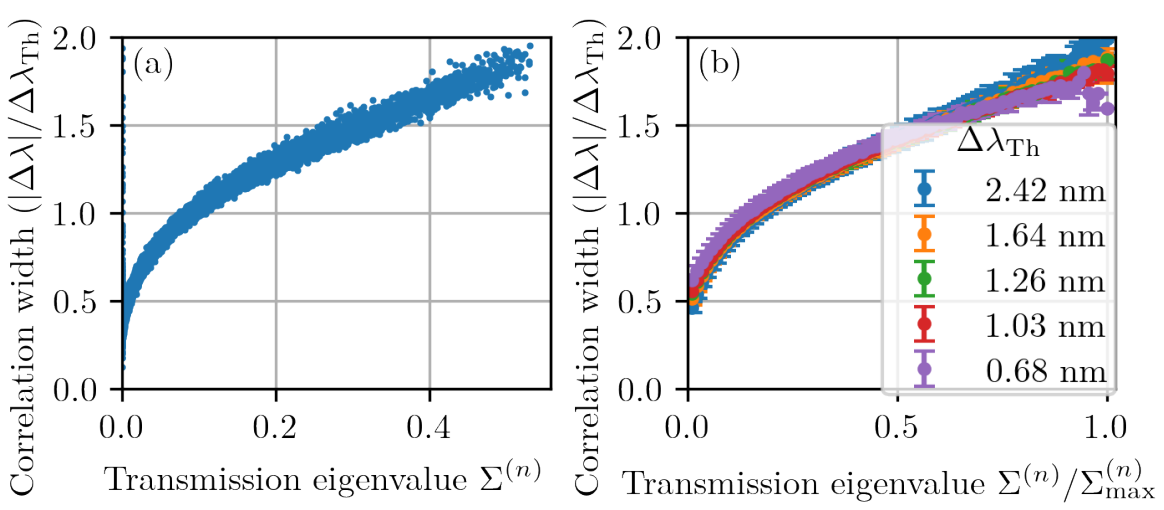

Figure 4.12.: Width of the $C^{(1)}$ correlation function as a function of the normalized transmittance. The width is normalized to the Thouless width. In (a) we plot the results for a 5.2- $\mu \mathrm{m}$-thick sample. In (b) we plot the results for different sample thickness, with the transmission eigenvalue normalized to their maximum transmission eigenvalue. The datapoints are binned over multiple measurement points, the errorbars indicate the standard deviation over these points. Each curve is derived from a dataset such as Fig 4.10.

\subsection{Conclusions}

In this chapter, we investigated the spectral behavior of transmission channels. We found three main results. First we found that the spectral shape of the transmittance of a transmission channel with above average transmittance is independent of the transmission of said sample and the thickness of the sample. Second we have found that the spectral shape of these transmission transmission channels can be described by the sum $\left(C^{(1)}+b C^{(2)}\right)$ with $b$ proportional to the matrix fidelity. Thirdly we have found the surprising result that transmission channels with the average sample transmittance increase in transmission from detuning.

Additionally, we have also investigated the correlation width of the transmission channels, which are in line with earlier results on a single channel.

These results show that the spectral behavior of the transmission of trans- 
mission channels is nontrivial and require new theoretical work for a better understanding of their behavior. 


\section{Bibliography}

[1] C. W. J. Beenakker, "Random-matrix theory of quantum transport," Rev. Mod. Phys. 69, 731-808 (1997).

[2] Y. V. Nazarov, "Limits of universality in disordered conductors," Phys. Rev. Lett. 73, 134-137 (1994).

[3] E. Akkermans and G. Montambaux, Mesoscopic Physics of Electrons and Photons (Cambridge University Press, 2007).

[4] O. N. Dorokhov, "On the coexistence of localized and extended electronic states in the metallic phase," Solid State Commun. 51, 381-384 (1984).

[5] P. A. Mello, P. Pereyra, and N. Kumar, "Macroscopic approach to multichannel disordered conductors," Ann. of Phys. 181, 290-317 (1988).

[6] J. B. Pendry, A. MacKinnon, and A. B. Pretre, "Maximal fluctuations - a new phenomenon in disordered systems," Physica A 168, 400-407 (1990).

[7] S. Rotter and S. Gigan, "Light fields in complex media: Mesoscopic scattering meets wave control," Rev. Mod. Phys. 89, 015005 (2017).

[8] D. A. B. Miller, "Waves, modes, communications, and optics: a tutorial," Adv. Opt. Photon. 11, 679-825 (2019).

[9] I. M. Vellekoop and A. P. Mosk, "Universal optimal transmission of light through disordered materials," Phys. Rev. Lett. 101, 120601 (2008).

[10] M. Kim, Y. Choi, C. Yoon, W. Choi, J. Kim, Q.-H. Park, and W. Choi, "Maximal energy transport through disordered media with the implementation of transmission eigenchannels," Nat. Photonics 6, 581-585 (2012).

[11] Z. Shi and A. Z. Genack, "Transmission eigenvalues and the bare conductance in the crossover to anderson localization," Phys. Rev. Lett. 108, 043901 (2012).

[12] B. Gérardin, J. Laurent, A. Derode, C. Prada, and A. Aubry, "Full transmission and reflection of waves propagating through a maze of disorder," Phys. Rev. Lett. 113, 173901 (2014).

[13] S. M. Popoff, A. Goetschy, S. Liew, A. Stone, and H. Cao, "Coherent control of total transmission of light through disordered media," Phys. Rev. Lett. 112, 133903 (2014).

[14] A. Peña, A. Girschik, F. Libisch, S. Rotter, and A. Chabanov, "The 
single-channel regime of transport through random media," Nat. Commun. 5, 3488 (2014).

[15] A. P. Mosk, A. Lagendijk, G. Lerosey, and M. Fink, "Controlling waves in space and time for imaging and focusing in complex media," Nat. Photonics 6, 283 (2012).

[16] J. Wang and A. Z. Genack, "Transport through modes in random media," Nature 471, 345-348 (2011).

[17] M. Davy, Z. Shi, J. Wang, X. Cheng, and A. Z. Genack, "Transmission eigenchannels and the densities of states of random media," Phys. Rev. Lett. 114, 033901 (2015).

[18] J. Bosch, S. A. Goorden, and A. P. Mosk, "Frequency width of open channels in multiple scattering media," Opt. Express 24, 26472-26478 (2016).

[19] Z. Shi and A. Z. Genack, "Dynamic and spectral properties of transmission eigenchannels in random media," Phys. Rev. B 92, 184202 (2015).

[20] C. W. Hsu, A. Goetschy, Y. Bromberg, A. D. Stone, and H. Cao, "Broadband coherent enhancement of transmission and absorption in disordered media," Phys. Rev. Lett. 115, 223901 (2015).

[21] S. Feng, C. Kane, P. A. Lee, and A. D. Stone, "Correlations and fluctuations of coherent wave transmission through disordered media," Phys. Rev. Lett. 61, 834-837 (1988).

[22] M. C. W. van Rossum and T. M. Nieuwenhuizen, "Multiple scattering of classical waves : microscopy , mesoscopy , and diffusion," Rev. Mod. Phys. 71, 313-371 (1999).

[23] R. Berkovits, M. Kaveh, and S. Feng, "Memory effect of waves in disordered systems: A real-space approach," Phys. Rev. B 40, 737-740 (1989).

[24] I. Freund, M. Rosenbluh, and S. Feng, "Memory effects in propagation of optical waves through disordered media," Phys. Rev. Lett. 61, 23282331 (1988).

[25] M. P. van Albada, J. F. de Boer, and A. Lagendijk, "Observation of longrange intensity correlation in the transport of coherent light through a random medium," Phys. Rev. Lett. 64, 2787-2790 (1990).

[26] F. Scheffold and G. Maret, "Universal conductance fluctuations of light," Phys. Rev. Lett. 81, 5800-5803 (1998).

[27] J. F. de Boer, M. P. van Albada, and A. Lagendijk, "Transmission 
and intensity correlations in wave propagation through random media," Phys. Rev. B 45, 658-666 (1992).

[28] I. M. Vellekoop, P. Lodahl, and A. Lagendijk, "Determination of the diffusion constant using phase-sensitive measurements," Phys. Rev. E 71, 056604 (2005).

[29] P. Pai, "Accurate statistics from optical transmission matrix measurements," PhD thesis, Universiteit Utrecht (2020).

[30] F. van Beijnum, E. G. van Putten, A. Lagendijk, and A. P. Mosk, "Frequency bandwidth of light focused through turbid media," Opt. Lett. 36, 373-375 (2011).

[31] A. Goetschy and A. D. Stone, "Filtering random matrices: The effect of incomplete channel control in multiple scattering," Phys. Rev. Lett. 111, 063901 (2013). 


\section{Time delay of transmission channels}

\subsection{Introduction}

Light pulses transmitted through a diffuse multiple scattering medium are scattered and transmitted as a stretched pulse with a complicated specklelike time-dependence. The average time delay of the transmitted light is related to the properties of the material, such as the density of states [1-3], the energy velocity $[4,5]$ and can indicate resonances $[6]$.

The time delay can be characterized with the Wigner-Smith [7-9] operator, which gives the time delay for scattering processes. It has been used to investigate time delay eigenstates and principal modes in microwave cavities, random media and multimode fibers [10-12]. Closely related is numerical work on the storage time of light in scattering media with the dwell-time operator [13-15]. Experiments on light in diffusing media show focusing of light at specific time delays through wavefront shaping [16-18]. The measurement of multispectral and time resolved transmission matrices [19-25], has opened up the possibility of measuring the time delay of transmission channels, however resolving both highly transmitting transmission channels and the time delay simultaneously has been unreported. The distribution of time delays of individual transmission channels in a multiple scattering medium as such is unknown. Random matrix theory of chaotic systems shows the time delay and the transmission operator to be uncorrelated [26,27], and as a result no strong correlation between the transmittance and the time delay is expected.

In this chapter, we tackle the open question whether the open channels are associated with specific time delays; is transport through open transmission channels different from the average time delay? To answer this question we measure the phase time delay of individual transmission channels. To find 
the time delay we measure a high-fidelity multispectral transmission matrix, which allows us to probe the phase time delay of channels for a wide range of transmission values. We numerically correct for phase drift between adjacent transmission matrices, which allows us to directly calculate the phase time delay of the transmission channels. Finally we compare the phase time delay with the Thouless time $\tau_{\text {Th }}$ which is a measure for time delay of a random wave [28].

\subsection{Coherent multispectral transmission matrix}

We calculate the transmission channels and their associated phase time delay from the multispectral transmission matrix. In Chapter 4 we describe the parameter choices for the transmission matrix measurement. Note that in this measurement procedure we correct for phase shift between matrices measured at different wavelengths. We expect to find a phase delay between transmission matrices measured at different wavelengths due to the diffuse delay time [29]. However, in our experiment we also measure a phase delay due to a path length difference between the reference path and the signal path, which we cannot separate out. Therefore we correct the phase shift between adjacently measured transmission matrices such that the average phase shift between adjacent transmission matrices equals zero. We also correct for any phase shift between the transmission matrices measured at the (same) shortest wavelength, by numerically rotating the elements in the transmission matrix in the complex plane: $T^{\prime}=T \exp \left\{i \phi_{T M 0,1}\right\}$, where $\phi_{T M 0,1}=\arg \left(\sum_{i, j} T_{i, j}^{*}\left(\lambda_{1}\right) T_{i, j}\left(\lambda_{0}\right)\right)$. As a result the phase delay we measure is a differential phase delay, with respect to the phase delay of a random incident wave. However the pulse delay of a random wave is well studied [30].

We correct for a phase shift $\phi_{T M 0,1}$ between spectrally adjacent matrices. This corrects for the intensity-weighted average phase delay of the matrix elements. If all elements of the transmission matrix were characterized by a single phase delay time, this correction would lead to a zero phase shift between all measured matrices. There is still a phase shift between transmission matrices at larger wavelength differences, due to the spread of delay times in the transmission matrix. In Fig. 5.1 we plot the phase shift versus detuning normalized by the Thouless width. We have defined the Thouless 

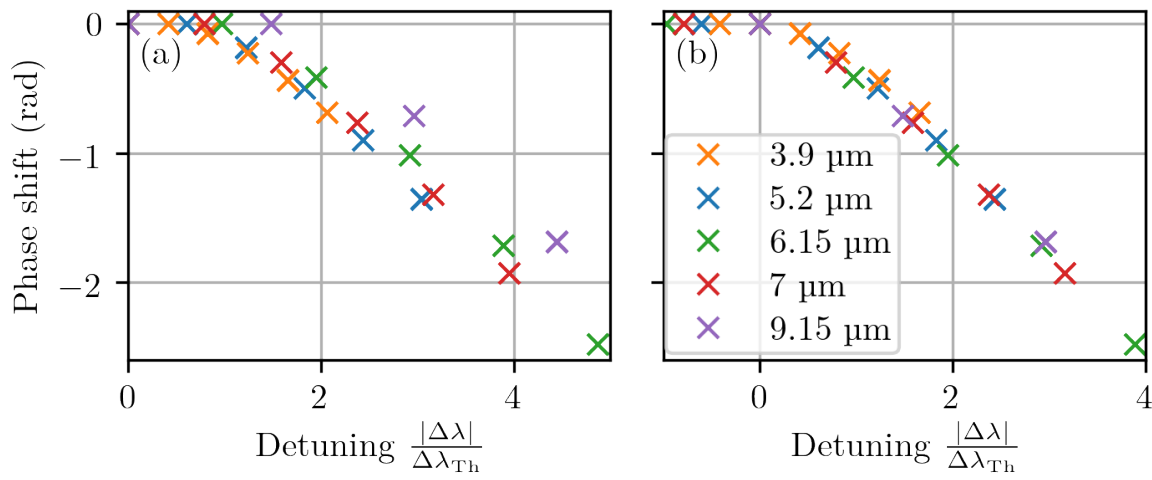

Figure 5.1.: Phase of the correlation between transmission matrices versus wavelength detuning $\frac{|\Delta \lambda|}{\Delta \lambda_{\mathrm{Th}}}$ for different sample thickness (a). In (b) phase shift versus detuning where we shift the individual curves such that the second point is shifted to zero detuning.

width as the wavelength difference for which the speckle correlation decays to one half, $C^{(1)}(\Delta \omega)=0.5$. In Fig. 5.1 (a) we plot the phase shift versus the detuning. The phase shift of the individual samples is approximately linear except for the first two points. We shift the curves such that the second point is shifted to zero detuning (b). We find that the differential phase is similar for the different samples and depends only on detuning relative to the Thouless width. This indicates that the transmission matrix cannot be characterized by a single well-defined phase delay time, but instead by a distribution of phase delay times.

\subsection{Transmission time delay of transmission channels}

Different measures of the time delays of light transmitted through a scattering material have been studied. In this section we look at the phase time delay of the transmission channels of a scattering medium, which we calculate from the multispectral transmission matrix of the system, which we measured in the spectral domain.

The time delay of a single channel system can be calculated from the phase 
time [7]:

$$
\tau_{\phi}(\omega)=\partial \phi(\omega) / \partial \omega
$$

where $\phi(\omega)$ indicates the phase of the wave. However, in our experiment we measure the time delay of a multichannel system. We use a variant to $\frac{\partial \phi}{\partial \omega}$ for a multichannel system. We write the transmitted field as $\boldsymbol{E}(\overline{\boldsymbol{k}}, \omega)=$ $\boldsymbol{A}(\overline{\boldsymbol{k}}, \omega) e^{i \phi(\omega)}$, we drop the $(\overline{\boldsymbol{k}} \omega)$ in the notation, and take the partial derivative with respect to $\omega$ on both sides, to arrive at,

$$
\frac{\partial \boldsymbol{E}}{\partial \omega}=\boldsymbol{A}^{\prime} e^{i \phi}+i \phi^{\prime} \boldsymbol{A} e^{i \phi}
$$

The phase function phi is chosen such that $A^{\prime}$ and $A$ are orthogonal, i.e., the non-orthogonal derivatives are included in the phase function. Next we take the inner product on both sides with the complex conjugate of $E(\omega)$, and we find $\sum_{\bar{k}} \boldsymbol{E}^{*} \frac{\partial \boldsymbol{E}}{\partial \omega}=\sum_{\bar{k}} i \boldsymbol{A}^{*} \boldsymbol{A} \phi^{\prime}+\sum_{\bar{k}} \boldsymbol{A}^{*} \boldsymbol{A}^{\prime}$, where we sum over all modes $\bar{k}$ of the vector. The second term is zero due to our choice of phi. We take the imaginary part: $\operatorname{Im} \sum_{\bar{k}} \boldsymbol{E}^{*} \frac{\partial \boldsymbol{E}}{\partial \omega}=\frac{\partial \phi}{\partial \omega} \sum_{\bar{k}} \boldsymbol{A}^{*} \boldsymbol{A}$ and rewrite,

$$
\frac{\partial \phi(\omega)}{\partial \omega}=\frac{\operatorname{Im} \sum_{\bar{k}} \boldsymbol{E}^{*} \frac{\partial \boldsymbol{E}}{\partial \omega}}{\sum_{\bar{k}}|\boldsymbol{E}|^{2}}
$$

We extract the time delay of the transmission channels in the following way. We pick two transmission matrices $T_{+}$and $T_{-}$with a wavelength detuning $\Delta \lambda$ and define $\bar{T}=\frac{1}{2}\left(T_{+}+T_{-}\right)$and $\bar{T}^{\prime}=\frac{1}{2}\left(T_{+}-T_{-}\right)$. We decompose the transmission matrix $\bar{T}$ into the transmission channels $\boldsymbol{v}^{(n)}$ and transmission eigenvalues $\Sigma^{(n)}$. Now we can easily calculate the propagated field by multiplying the transmission channel with the transmission matrix $\boldsymbol{E}^{*}=\left(\bar{T} \boldsymbol{v}^{(n)}\right)^{*}$ and calculate the derivative by multiplying the transmission channel with $\bar{T}^{\prime}: \frac{\partial \boldsymbol{E}}{\partial \omega}=\bar{T}^{\prime} \boldsymbol{v}^{(n)} \frac{1}{\Delta \lambda} \frac{\partial \lambda}{\partial \omega}$. The relative time delay $\Delta \tau_{\phi}$ which is defined as $\Delta \tau_{\phi}=\tau_{\phi}-\tau_{\mathrm{Th}}$, is now

$$
\Delta \tau_{\phi}=\frac{\partial \phi(\omega)}{\partial \omega}=\frac{\operatorname{Im}\left(\left(\bar{T} \boldsymbol{v}^{(n)}\right)^{*} \cdot \bar{T}^{\prime} \boldsymbol{v}^{(n)}\right)}{\left|\bar{T} \boldsymbol{v}^{(n)}\right|^{2}} \frac{1}{\Delta \lambda} \frac{\partial \lambda}{\partial \omega}
$$

In Fig. 5.2 we plot the relative time delay $\Delta \tau_{\phi}$ versus transmission eigenvalue $\Sigma^{(n)}$, measured on the $5.2 \mu \mathrm{m}$ sample, using a wavelength spacing between the transmission matrices $\Delta \lambda / \Delta \lambda_{\mathrm{Th}}=0.61$. We find the same distribution of time delay values when calculating the time delay from two independently measured transmission matrices, except for the transmission 


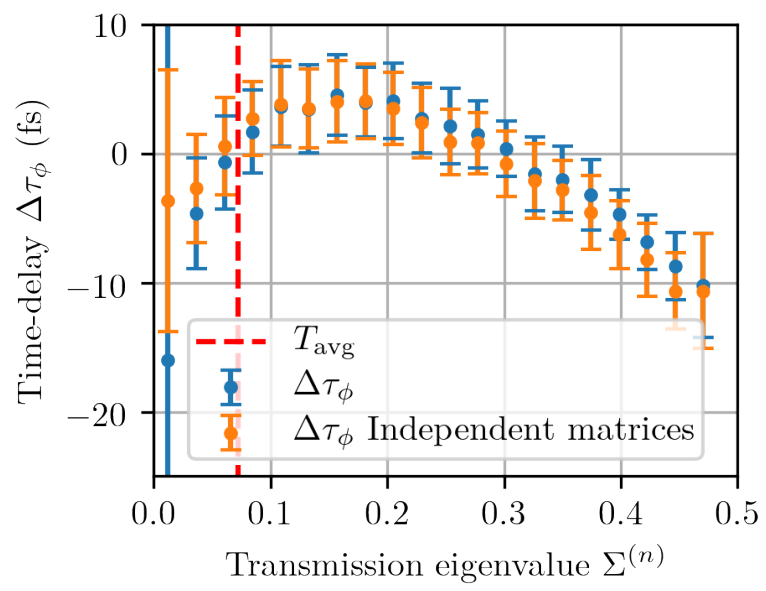

Figure 5.2.: Time delay $\Delta \tau_{\phi}$ relative to the Thouless time of transmission channels versus transmission eigenvalue $\Sigma^{(n)}$. In blue we plot the time delay calculated from the same matrices as the transmission channels, in orange calculated from independently measured transmission matrices. The results are binned according to transmission eigenvalue, error bars correspond to the standard deviation of the time delay within the bin. The average transmittance $T_{\text {avg }}$ is plotted in red.

channels with a transmittance below 0.01 , which have a large spread in time delay for both measurements. For random input vectors we find an average relative time delay of $0 \mathrm{fs}$ with a standard deviation of $4 \mathrm{fs}$, for reference $1 \mathrm{\mu m}$ of propagation of light through air takes $3.3 \mathrm{fs}$. To exclude possible measurement artifacts we propagate the transmission channels through a transmission matrix measured for a different sample so that the transmission matrix is fully uncorrelated. In this reference measurement we find no correlation between the time delay and the transmittance of the channel. The fact that the correlation is absent in the reference measurement indicates that the time delay is due to properties of the transmission channels, and is not an artifact of the measurement apparatus.

There is clearly a dependence of the time delay of a transmission channel on its transmission eigenvalue. The transmission channels with a transmittance 
just over the average transmittance have a slightly positive delay. For higher transmittance values the delay time drops, and the channels with the highest transmittance have a time delay of about $10 \mathrm{fs}$ less than the average time delay. We note that for all channels $\Delta \tau_{\phi}$ is small compared to the Thouless time $\tau_{\mathrm{Th}}$, which for this sample is $190 \mathrm{fs}$. We calculate the Thouless time $\tau_{\mathrm{Th}}$ from the measured Thouless width:

$$
\Delta \tau_{\phi}(\omega)=\frac{\lambda^{2}}{2 \pi c \Delta \lambda_{\mathrm{Th}}} .
$$

These results demonstrate that there is a measurable difference in time delay between transmission channels with different transmittance, of the order of $10 \%$ of the average.

As a consistency check we also calculate the time delay of the transmission channels propagated at different wavelength spacing $\Delta \lambda$ than where they are calculated at, i.e. we calculate the transmission channels from $\bar{T}_{\Delta \lambda=1 \mathrm{~nm}}$ and calculate $\Delta \tau_{\phi}$ for those channels also at $\Delta \lambda=3 \mathrm{~nm}$ and $\Delta \lambda=5 \mathrm{~nm}$ with their respective $\bar{T}$ and $\bar{T}^{\prime}$. In Fig. 5.3 we plot the time delay $\Delta \tau_{\phi}$. We find again that transmission channels with a transmittance above 0.2 slightly decrease in time delay. For the transmission channels with a lower transmittance we see a different behaviour. The cause of the deviation in behaviour at low transmittance is not clear.

We now measure the time delay for the transmission channels of samples with different thickness. In Fig. 5.4 we plot the time delay versus normalized transmission eigenvalue $\Sigma^{(n)} / \Sigma_{\max }^{(n)}$ for different sample thickness calculated with a $1 \mathrm{~nm}$ spectral spacing between the transmission matrices. We find the same behavior for all curves. We note that the Thouless width varies widely between the different samples. Nevertheless, the time delay versus transmittance shows a remarkable resemblance. 


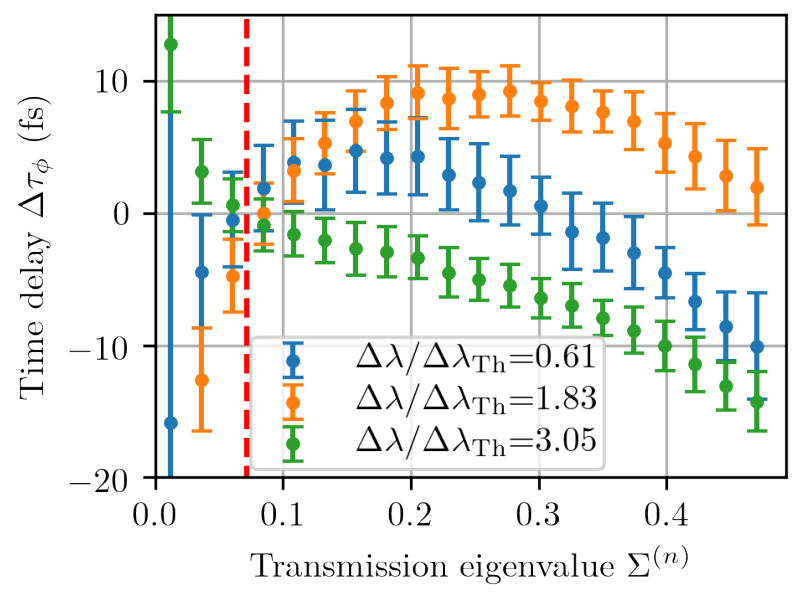

Figure 5.3.: Relative time delay of transmission channels versus transmittance. The transmission channels extracted from the average of two transmission matrices with $\Delta \lambda=1 \mathrm{~nm}$. In blue we plot the time delay extracted from the average of two transmission matrices with $1 \mathrm{~nm}$ detuning, in orange $3 \mathrm{~nm}$ and in green $5 \mathrm{~nm}$. Vertical dashed line: channel average transmittance. The average transmittance for the transmission matrix is plotted in red. Data is measured on a 5.2- $\mu$ m-thick sample. 


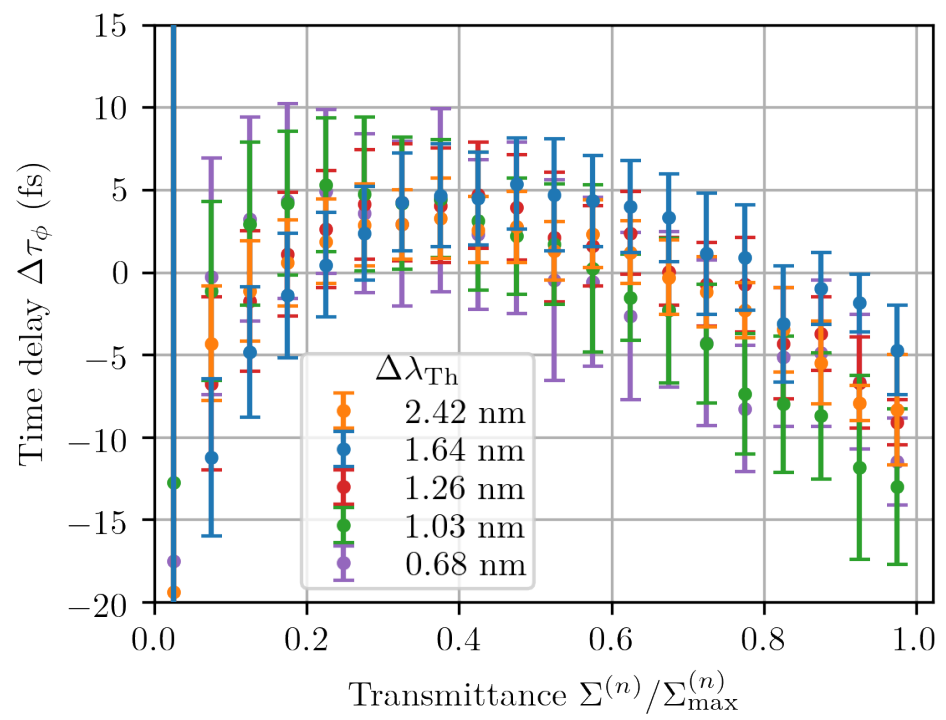

Figure 5.4.: Relative time delay of transmission channels versus transmission eigenvalue for different sample thicknesses, labeled by their Thouless width. All derivatives are taken with a transmission matrix spacing of $1 \mathrm{~nm}$. 


\subsection{Conclusions}

In this chapter, we have presented the first optical measurements of the time delay of the transmission channels of a multiple scattering medium. Our measurements are based on multispectral transmission matrix measurements with high fidelity measured with a monochromatic wavelength tunable light source. We find a consistent and reproducible dependence of the delay-time on the transmittance and that the most open channels are up to 15 femtoseconds shorter than the channels with slightly above average transmission, which experience the longest time delay. We find that the difference in time delay is small compared to the Thouless time, which is a measure for the average delay time. 


\section{Bibliography}

[1] V. Lyuboshitz, "On collision duration in the presence of strong overlapping resonance levels," Phys. Lett. B 72, 41 - 44 (1977).

[2] A. Yamilov and H. Cao, "Density of resonant states and a manifestation of photonic band structure in small clusters of spherical particles," Phys. Rev. B 68, 085111 (2003).

[3] M. Davy, Z. Shi, J. Wang, X. Cheng, and A. Z. Genack, "Transmission eigenchannels and the densities of states of random media," Phys. Rev. Lett. 114, 033901 (2015).

[4] M. P. van Albada, B. A. van Tiggelen, A. Lagendijk, and A. Tip, "Speed of propagation of classical waves in strongly scattering media," Phys. Rev. Lett. 66, 3132-3135 (1991).

[5] A. Lagendijk and B. A. van Tiggelen, "Resonant multiple scattering of light," Physics Reports 270, 143 - 215 (1996).

[6] J. Wang and A. Z. Genack, "Transport through modes in random media," Nature 471, 345-348 (2011).

[7] E. P. Wigner, "Lower limit for the energy derivative of the scattering phase shift," Phys. Rev. 98, 145-147 (1955).

[8] F. T. Smith, "Lifetime matrix in collision theory," Phys. Rev. 118, 349-356 (1960).

[9] P. Ambichl, "Coherent wave transport: time-delay and beyond," $\mathrm{PhD}$ thesis, Technische Universität Wien, Vienna (2016).

[10] S. Rotter, P. Ambichl, and F. Libisch, "Generating particlelike scattering states in wave transport," Phys. Rev. Lett. 106, 120602 (2011).

[11] J. Carpenter, B. J. Eggleton, and J. Schröder, "Observation of eisenbudwigner-smith states as principal modes in multimode fibre," Nat. Photonics 9, 751 (2015).

[12] P. Ambichl, A. Brandstötter, J. Böhm, M. Kühmayer, U. Kuhl, and S. Rotter, "Focusing inside disordered media with the generalized wigner-smith operator," Phys. Rev. Lett. 119, 033903 (2017).

[13] B. A. van Tiggelen, A. Tip, and A. Lagendijk, "Dwell times for light and electrons," J. Phys. A: Math. Gen. 26, 1731-1748 (1993).

[14] A. Lagendijk and B. A. van Tiggelen, "Accumulation of dwell times in light diffusion," Physica A: Statistical Mechanics and its Applications 200, 433 - 442 (1993). 
[15] M. Durand, S. M. Popoff, R. Carminati, and A. Goetschy, "Optimizing light storage in scattering media with the dwell-time operator," Phys. Rev. Lett. 123, 243901 (2019).

[16] J. Aulbach, B. Gjonaj, P. M. Johnson, A. P. Mosk, and A. Lagendijk, "Control of light transmission through opaque scattering media in space and time," Phys. Rev. Lett. 106, 103901:1 -4 (2011).

[17] O. Katz, E. Small, Y. Bromberg, and Y. Silberberg, "Focusing and compression of ultrashort pulses through scattering media," Nat. Photonics 5, 372-377 (2011).

[18] D. J. McCabe, A. Tajalli, D. R. Austin, P. Bondareff, I. A. Walmsley, S. Gigan, and B. Chatel, "Spatio-temporal focusing of an ultrafast pulse through a multiply scattering medium," Nat. Commun. 2, 447 (2011).

[19] Y. Choi, T. R. Hillman, W. Choi, N. Lue, R. R. Dasari, P. T. C. So, W. Choi, and Z. Yaqoob, "Measurement of the time-resolved reflection matrix for enhancing light energy delivery into a scattering medium," Phys. Rev. Lett. 111, 243901 (2013).

[20] S. Kang, S. Jeong, W. Choi, H. Ko, T. D. Yang, J. H. Joo, J.-S. Lee, Y.-S. Lim, Q.-H. Park, and W. Choi, "Imaging deep within a scattering medium using collective accumulation of single-scattered waves," Nat. Photonics 9, 253 (2015).

[21] D. Andreoli, G. Volpe, S. Popoff, O. Katz, S. Grésillon, and S. Gigan, "Deterministic control of broadband light through a multiply scattering medium via the multispectral transmission matrix," Sci. Rep. 5, 10347 (2015).

[22] M. Mounaix, D. Andreoli, H. Defienne, G. Volpe, O. Katz, S. Grésillon, and S. Gigan, "Spatiotemporal coherent control of light through a multiple scattering medium with the multispectral transmission matrix," Phys. Rev. Lett. 116, 253901 (2016).

[23] R. French, S. Gigan, and O. L. Muskens, "Speckle-based hyperspectral imaging combining multiple scattering and compressive sensing in nanowire mats," Opt. Lett. 42, 1820-1823 (2017).

[24] J. Carpenter, B. J. Eggleton, and J. Schröder, "Complete spatiotemporal characterization and optical transfer matrix inversion of a 420 mode fiber," Opt. Lett. 41, 5580-5583 (2016).

[25] M. Mounaix, H. Defienne, and S. Gigan, "Deterministic light focusing in space and time through multiple scattering media with a time-resolved 
transmission matrix approach," Phys. Rev. A 94, 041802 (2016).

[26] P. W. Brouwer and M. Büttiker, "Charge-relaxation and dwell time in the fluctuating admittance of a chaotic cavity," Europhys. Lett. 37, 441-446 (1997).

[27] Y. V. Fyodorov and D. V. Savin, "Resonance scattering of waves in chaotic systems," in "The Oxford Handbook of Random Matrix Theory," , G. Akemann, J. Baik, and P. Di Francesco, eds. (Oxford University Press, Oxford, 2011), chap. 34.

[28] D. J. Thouless, "Maximum metallic resistance in thin wires," Phys. Rev. Lett. 39, 1167-1169 (1977).

[29] I. M. Vellekoop, P. Lodahl, and A. Lagendijk, "Determination of the diffusion constant using phase-sensitive measurements," Phys. Rev. E 71, 056604 (2005).

[30] P. M. Johnson, A. Imhof, B. P. J. Bret, J. G. Rivas, and A. Lagendijk, "Time-resolved pulse propagation in a strongly scattering material," Phys. Rev. E 68, 016604 (2003). 


\section{Measurements of time delay eigenstates of light transmitted through scattering media}

\subsection{Introduction}

The time delay of light transmitted through scattering systems determines how long light interacts with a sample, and as such is important for various processes such as spectroscopy, pulse transmission, and energy conversion. The most extreme values of the time delay can be found by eliciting the time delay eigenstates, which are the eigenstates of the Wigner-Smith time delay operator $[1,2]$. These are states that scatter with a well-defined time delay.

Measurements on the time delay eigenstates have been performed in acoustics in two dimensions, studying vibrations on a membrane and in microwave waveguide measurements, in which particle like modes can be found $[3,4]$. Optical measurements have been performed in multimode fibers with strong mode coupling, where principal modes with well-defined delay times are found that exhibit an increased spectral correlation width $[5,6]$.

Simulations on light in scattering media demonstrate the existence of branched light instead of a spreading of the light [7]. Numerical simulations of the dwell time operator in multiple scattering media [8], indicate the existence of states with long delay and dwell time.

Experiments with multispectral transmission matrices and wavefront shaping demonstrate control of the pulse duration and the average time delay of light transmitted through a scattering medium [9-15], but have not looked at time delay eigenstates.

In this chapter, we determine the time delay eigenvalues and eigenstates from a measurement of the transmission section of the reduced Wigner-Smith 
matrix $[1,2,4,7,16,17]$. We compare different ways to calculate the time delay, investigate how measurement choices influence the measured time delay eigenvalues, and finally we investigate the transmission properties of the time delay eigenstates such as the transmittance and their spectral correlations.

\subsection{Theory}

To describe the time behavior of scattering events, there are various times that are defined in literature [1,18-21]. For single channel scattering we use the Wigner phase delay time defined by Wigner and Eisenbud $[1,18]$. The Wigner phase delay time is the energy derivative of the scattering phase shift $\tau_{\phi}(E)=\hbar \partial \phi / \partial E$. In optics, this can be easily computed as the frequency derivative of the phase $\tau_{\phi}(\omega)=\partial \phi(\omega) / \partial \omega$. Smith introduced the concept of the Wigner-Smith time delay matrix $\hat{Q}$, which generalizes the Wigner phase delay time to a multi-channel system [2]. The Wigner-Smith matrix $\hat{Q}$ is calculated from the unitary scattering matrix $S$,

$$
\hat{Q}=-i \hbar S^{-1} \frac{\partial S}{\partial E}
$$

where we note that $S^{-1}=S^{\dagger}$ because $S$ is unitary.

The Wigner-Smith time delay matrix can be generalized to a matrix that only operates on the transmission matrix $[4,7,16,17]$, which allows us to calculate a reduced Wigner-Smith time delay matrix from two transmission matrices $T_{+}$and $T_{-}$measured at $\Delta \lambda$. We calculate the reduced time delay operator $Q$ according to same method as Brandstötter [7],

$$
Q=-i \bar{V} \bar{\Sigma}^{-1} \bar{U}^{\dagger} \bar{U} \bar{U}^{\dagger} \frac{\partial T}{\partial \omega} \bar{V} \bar{V}^{\dagger}
$$

where $\bar{U}, \bar{V}$ and $\bar{\Sigma}$ are the truncated matrices from the singular value decomposition of $\bar{T}=\frac{1}{2}\left(T_{+}+T_{-}\right)$, where we truncate the rows and columns corresponding to $\sigma<\sigma_{\max } \eta$, where $\eta$ denotes the cut-off value. We calculate $\frac{\partial T}{\partial \omega}=\left(T_{+}-T_{-}\right) \frac{1}{\Delta \lambda} \frac{\partial \lambda}{\partial \omega}$.

The time delay eigenstates $\boldsymbol{q}^{(n)}$ are the eigenvectors of $Q$. Due to the non-Hermitian nature of $Q$, the reduced time delay operator $Q$ has complex eigenvalues, whereas the eigenvalues of $\hat{Q}$ are real. The real part of the eigenvalues $w$ corresponds to the Wigner delay time, as seen in our experiments 
and in the microwave work by Böhm [4]. A derivation with a didactic approach can be found in the $\mathrm{PhD}$ thesis by Ambichl [16]. In the experimental part of this chapter we will look at differences between different methods of measuring time delay.

\subsection{Measurement of time delay eigenstates}

In this section, we look at the time delay eigenstates of multiple scattering media. We find the time delay eigenstates by eigenvalue decomposition of the reduced time delay operator $Q$, which we obtain in turn from the multi spectral transmission matrix. The parameter choices for the multi spectral transmission matrix measurement are described in Chapter 4. We use a set of five different transmission matrices measured at different sample thicknesses and Thouless widths. We compare different methods to measure the time delay and look at the dependence of the time delay eigenvalues on the sample thicknesses.

The eigenvalues $w$ of the matrix $Q$ are sensitive to measurement noise in the transmission matrix. We obtain an independent measurement of time delay operator $Q^{\prime}$ from two independently measured transmission matrices $T_{+}^{\prime}$ and $T_{-}^{\prime}$, measured at the same position. From $Q^{\prime}$ we calculate the expectation value of the time delay by multiplying the time delay eigenstate $q^{(n)}$ with the time delay operator $Q^{\prime}$, and then taking the Hermitian inner product with the time delay eigenstate $q^{(n)}$ to calculate the expectation value: $w^{\prime}=\left(\boldsymbol{q}^{(n)} \cdot Q^{\prime} \boldsymbol{q}^{(n)}\right)$. Additionally we calculate the phase time delay $\tau_{\phi}$ directly from a spectral derivative. In this way we have three methods to calculate the time delay of an eigenstate: the eigenvalue $w$, the expectation value $w^{\prime}$, and the phase time delay $\tau_{\phi}$. First, we look at the eigenvalues $w$ and the influence of the measurement noise by comparison with the expectation value $w^{\prime}$ calculated from propagation through an independent set of matrices.

In Fig. 6.1, we plot the eigenvalues $w$ and $w^{\prime}$ in units of the Thouless time, a 2.7-um-thick sample and with a wavelength spacing of $1 \mathrm{~nm}$. We use a cut-off value $\eta=0.07$ in the reduced time delay operator throughout this chapter.

We find that the eigenvalues have a large imaginary component, due to 


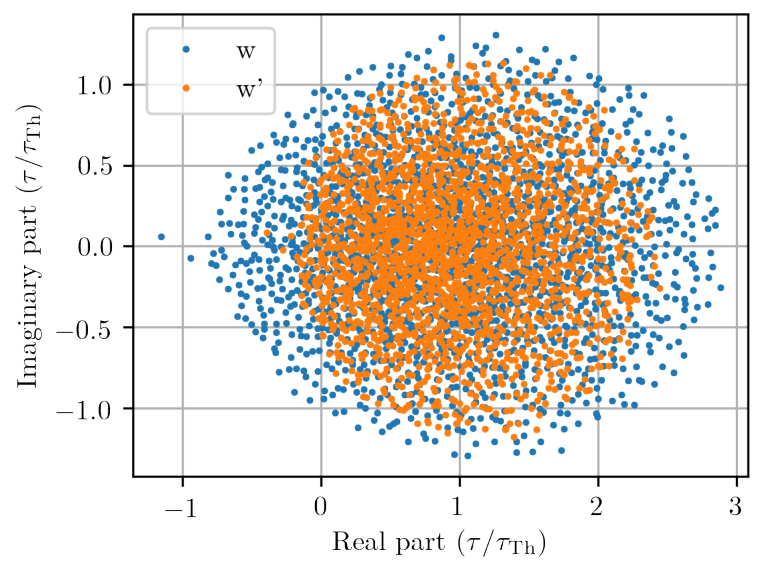

Figure 6.1.: Blue: measured complex eigenvalue $w$ of the time delay operator $Q$. Orange: corresponding time delay expectation value $w^{\prime}$ calculated from independently measured time delay operator $Q^{\prime}$. The eigenvalues are scaled according to $w / \tau_{\text {Th }}$ and calculated for a 2.7- $\mu \mathrm{m}$-thick sample.

the non-Hermiticity of the reduced time delay operator. The eigenvalue distribution is close to circular symmetric, with a slight elongation along the real axis. The distribution of the time delay expectation values $w^{\prime}$ is more circular symmetric. We find a range of time delay eigenvalues from $w^{\prime}$ from -0.4 to 2.4 times the Thouless time.

In Fig. 6.2, we plot the histogram of the time delay expectation value $\operatorname{Re}\left(w^{\prime}\right)$ for the $2.7-\mu m$-thick sample. The histogram summarizes the time delay values of five different matrix pairs, all using a wavelength step size of $1 \mathrm{~nm}$. We find a slight asymmetry in the histogram with a tail towards longer time delays and a sharp cutoff for negative time delays.

The real part of the time delay eigenvalues gives us the proper time delay [16]. We compare the real part of time delay eigenvalues $\operatorname{Re}(w)$ with real part of the time delay expectation value $\operatorname{Re}\left(w^{\prime}\right)$ and the phase time delay $\tau_{\phi}$. This gives us a comparison with the results that we have obtained earlier for the time delay of transmission channels, and it shows how sensitive we are to phase noise. We have derived before that we can calculate the time delay 


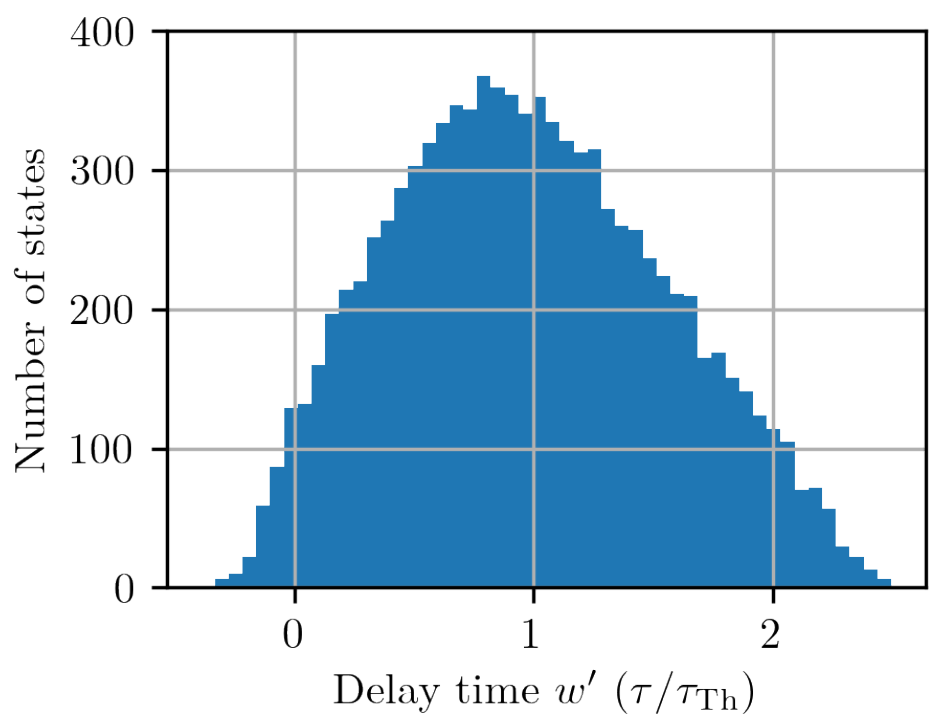

Figure 6.2.: Histogram of time delay expectation value $\operatorname{Re}\left(w^{\prime}\right)$ for the 2.7-um-thick sample. The histogram is calculated for time delay over five different matrix pairs, all calculated with a wavelength stepsize of $1 \mathrm{~nm}$.

$\tau_{\phi}$ (Eq. (5.4)) with the spectral derivative of the transmission matrix $T^{\prime}$. We calculate the phase time delay $\tau_{\phi}$ by multiplying the eigenstate $\boldsymbol{q}^{(n)}$ with the transmission matrix $\bar{T}$ and with the derivative of the transmission matrix $\bar{T}^{\prime}$. We take the inner product between the two resultant vectors, and divide the real part by the norm of the vector multiplied with the transmission matrix. We multiply by $\frac{\partial \lambda}{\partial \omega}$ to calculate the time delay as

$$
\tau_{\phi}=\frac{\operatorname{Im}\left(\left(\bar{T} \boldsymbol{q}^{(n)}\right)^{*} \cdot \bar{T}^{\prime} \boldsymbol{q}^{(n)}\right)}{\left|\bar{T} \boldsymbol{q}^{(n)}\right|^{2}} \frac{1}{\Delta \lambda} \frac{\partial \lambda}{\partial \omega}+\tau_{\mathrm{Th}} .
$$

In Fig. 6.3, we plot the time delay $\tau_{\phi}$ and the time delay expectation value $\operatorname{Re}\left(w^{\prime}\right)$ versus the time delay eigenvalue $\operatorname{Re}(w)$. We find that the time delay $\tau_{\phi}$ is about $20 \%$ higher than the time delay expectation value $\operatorname{Re}\left(w^{\prime}\right)$. However, both give a similar representation of the time delay.

An important parameter in calculating the time delay eigenvalues is the cut-off value $\eta$ that is chosen when calculating the pseudoinverse. The time 


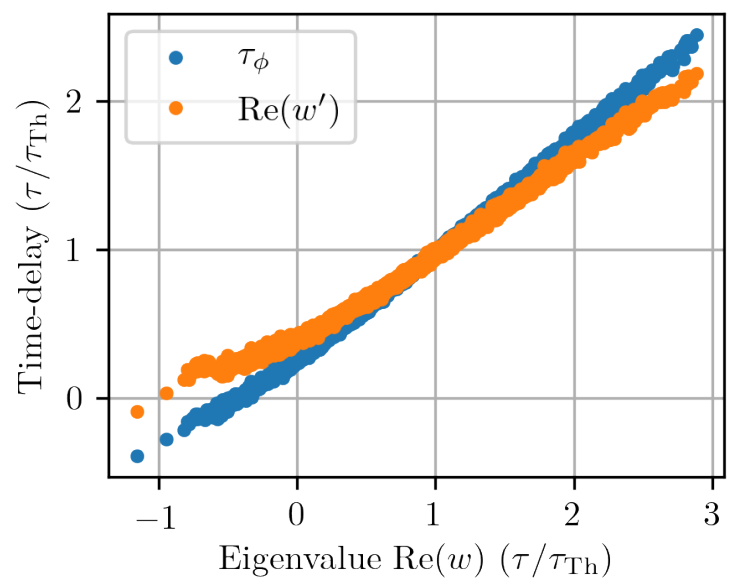

Figure 6.3.: Time delay $\tau / \tau_{\text {Th }}$ versus time delay eigenvalue $\operatorname{Re}(w)$. Blue: phase time delay $\tau_{\phi}$, orange: time delay expectation value $\operatorname{Re}\left(w^{\prime}\right)$. The data is calculated for a 2.7- $\mu$ m-thick $\mathrm{ZnO}$ sample, with a wavelength step of $1 \mathrm{~nm}$ and a Thouless width of $2.42 \mathrm{~nm}$.

delay eigenvalue distribution is dependent on the cut-off value $\eta$. We look at the maximum and minimal values that we find for the time delay eigenvalues, and find the dependence on $\eta$. In Fig. 6.4, we plot the maximum and minimum values of the phase time delay $\tau_{\phi}$ and the time delay expectation value $\operatorname{Re}\left(w^{\prime}\right)$ versus the cut-off value $\eta$. We also mark the cut-off value $\eta=0.07$. The data was processed from the transmission matrices of a $2.7 \mu \mathrm{m}$ sample, with a wavelength spacing of $1 \mathrm{~nm}$ and a Thouless width of $2.42 \mathrm{~nm}$.

We find that the maximum and minimum value of the phase time delay $\tau_{\phi}$ and the time delay expectation value $\operatorname{Re}\left(w^{\prime}\right)$ depend strongly on the cut-off value $\eta$, and both values show the same behavior. The maximum time delay sharply decreases for cut-off values $\eta<0.05$, and gradually decreases for cut-off values $\eta>0.08$. 


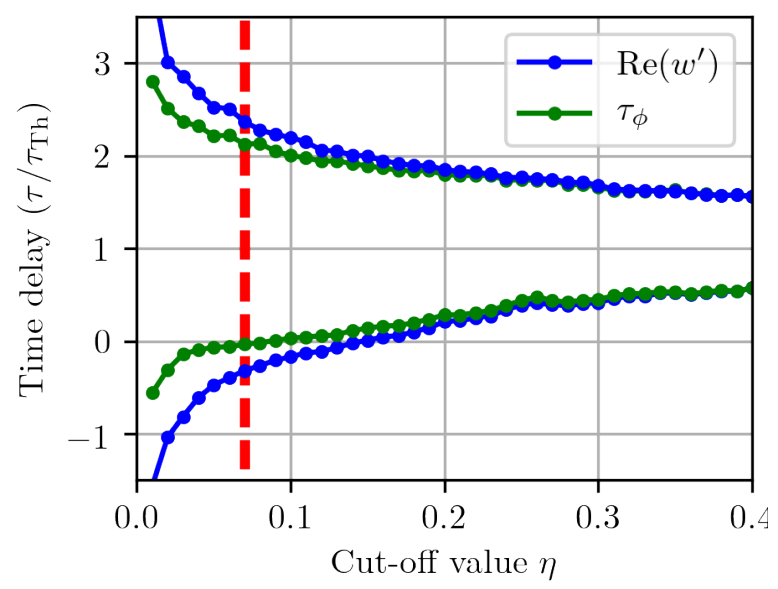

Figure 6.4.: Highest and lowest time delay $\tau / \tau_{\phi}$ versus cut off values $\eta$. In red we indicate a value $\eta=0.07$. We plot both the time delay expectation value $\operatorname{Re}\left(w^{\prime}\right)$ and the phase delay time $\tau_{\phi}$.

\subsection{Consistency checks}

\section{Average-time delay}

As a consistency check, we investigate the influence of the average-time delay on the time delay eigenvalues by rotating the matrix values of $T_{+}$in the complex plane to artificially change the average time delay: $T_{+}^{\prime}=T_{+} \exp \left(j d \phi_{\mathrm{Th}}\right)$, where $\phi_{\mathrm{Th}}$ is the phase shift corresponding to the Thouless time, and $d$ a real constant corresponding to the delay relative to the Thouless time. We calculate the eigenvalues, and compare the histogram for different values of delay $d$. In Fig. 6.5 we show the histogram of the time delay eigenvalues $w^{\prime} / \tau_{\text {Th }}$ for different values of $d$, where we have subtracted $d \phi_{\text {Th }}$ from the time delay $w^{\prime}$. We find the same distribution of eigenvalues for different values of $d$. This shows that the time delay eigenvalues are independent of any additional average phase shift between $T_{+}$and $T_{-}$and hence that our results are insensitive to a possible measurement error in the Thouless time. 


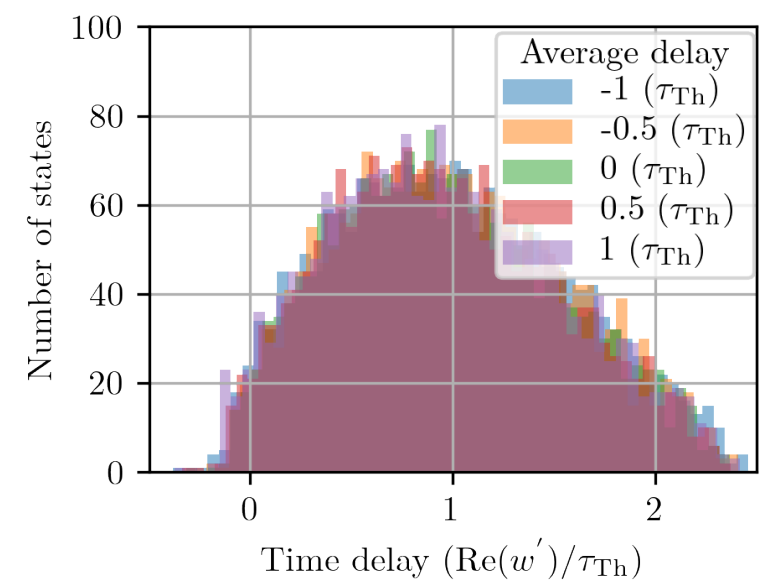

Figure 6.5.: Time delay $w^{\prime} / \tau_{\text {Th }}$ histogram for different average time delays. The datasets have been shifted by their average time delay to overlap the distributions. Data calculated on a 5.2- $\mu$ m-thick sample.

\section{Transmission matrix spacing}

Finally, we investigate in how far the calculated time delay eigenvalues are also dependent on the spacing between the matrices that are used to calculate $Q$. In order to find the influence of the spacing on the time delay eigenvalues, we calculate the time delay eigenvalues for the same sample thickness, at different matrix spacing. In order to cover a large range of matrix spacings relative to the Thouless frequency, we calculate the time delay eigenvalues for a thin sample with a thickness of $2.7 \mu \mathrm{m}$. The transmission matrices are measured at six wavelengths spaced at $1 \mathrm{~nm}$, which at this sample thickness corresponds to a spacing of 0.41 Thouless widths.

In Fig. 6.6, we present a histogram of the real part of the time delay expectation time $\operatorname{Re}\left(w^{\prime}\right)$ for different transmission matrix spacing on the same sample. We find that the histogram of time delay values is asymmetric and broad if the spacing between the matrices used to take the derivatives is smaller than the Thouless width. For larger spacings, the histogram becomes narrower and more symmetric. However, it is unlikely that such large spacings give a good representation of the derivative. The number of number of 


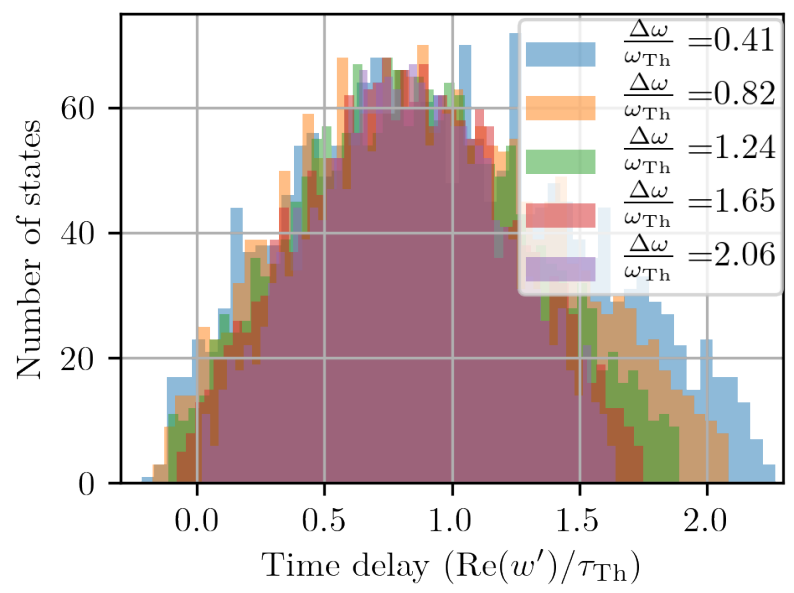

Figure 6.6.: Histogram of the time delay $\operatorname{Re}\left(w^{\prime}\right) / \tau_{\text {Th }}$ calculated with different transmission matrix spacings. All distributions are calculated from the same 2.7- $\mu$ m-thick $\mathrm{ZnO}$ sample.

time delay eigenstates in the histogram depends on the spacing. This is because we use a fixed cut-off value $\eta$ for calculating the time delay eigenstates. At a large spacing, we calculate the transmission eigenvalues of the average of two decorrelated transmission matrices, which are lower than those of two highly correlated transmission matrices, causing more of them to fall below the threshold.

\subsection{Properties of time delay eigenstates}

In this section, we look at the time delay eigenstates of different sample thickness and the intensity transmittance versus the eigenvalue. Finally we have a look at the spectral properties of the time delay eigenstates, and we look at how the propagated time delay eigenstates correlate as a function of wavelength detuning.

We calculate the time delay eigenstates for different sample thicknesses from the transmission matrices with a $1 \mathrm{~nm}$ spectral spacing. Due to the difference in sample thickness the spacing between the matrices relative to the Thouless frequency is different for the different sample thicknesses. As 


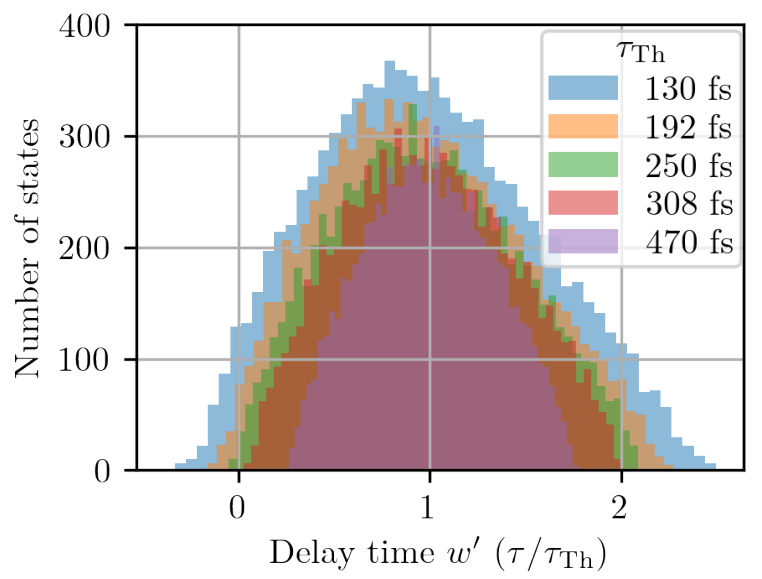

Figure 6.7.: Histogram of time delay expectation value $\operatorname{Re}\left(w^{\prime}\right)$ for different samples, labeled by their Thouless time $\tau_{\text {Th }}$. Every histogram comprises 5 independent sets of transmission data, calculated with a wavelength stepsize of $1 \mathrm{~nm}$.

we have seen, this will influence the distribution of eigenvalues. We use the same cut-off value $\eta=0.07$

In Fig. 6.7 we plot a histogram of the time delay expectation value $\operatorname{Re}\left(w^{\prime}\right)$ for different sample thickness. For every sample thickness, we calculate the histogram over 5 different sets of transmission matrix pairs with a $1 \mathrm{~nm}$ frequency spacing for every sample thickness. We find that the shape of the time delay eigenvalue histogram is similar for different sample thickness, however for the thin samples we find a larger spread of time delays. This can be explained by the fact that the eigenstates are calculated at a different spectral spacing relative to the Thouless frequency, which causes a change in the shape of the eigenvalue distribution, as we have seen in Fig. 6.6. A list of the sample properties i.e. thickness, Thouless width and Thouless time is found in Table 6.1.

We calculate the transmittance $T_{\boldsymbol{q}^{(n)}}$ of the time delay eigenstates $\boldsymbol{q}^{(n)}$ by multiplying the states with the transmission matrix to calculate the propagated field, and calculating the vector norm $T_{\boldsymbol{q}^{(n)}}=\left|\bar{T} \boldsymbol{q}^{(n)}\right|^{2}$.

In Fig. 6.8, we plot the transmittance $T_{\boldsymbol{q}^{(n)}}$ versus the time delay $\operatorname{Re}\left(w^{\prime}\right) / \tau_{\text {Th }}$ 


\begin{tabular}{|l|l|l|l|l|l|}
\hline Physical thickness $(\mu \mathrm{m})$ & 2.7 & 5.2 & 10.8 & 8.9 & 15.6 \\
\hline Thouless width $(\mathrm{nm})$ & 2.42 & 1.64 & 1.26 & 1.03 & 0.68 \\
\hline Thouless time $(\mathrm{fs})$ & 130 & 192 & 250 & 308 & 470 \\
\hline
\end{tabular}

Table 6.1.: List of samples and properties. These are the same samples as in Table 4.1.

for the time delay eigenstates. We find that all time delay eigenstates have a transmittance below the average sample transmittance. For any time delay the transmittance values show a large spread. The maximum transmittance occurs for time delay eigenstates around the Thouless time. The naive expectation would be that there is no correlation between the transmittance of a time delay eigenstate and the delay time, because we find no correlation between the delay time of a transmission channel and their delay time (Chapter 5). However this is not the case.

\subsubsection{Correlation width of time delay eigenstates}

For the propagation of short pulses through time delay eigenstates it is important how much the transmitted wavefront changes as a function of wavelength, as this random dispersion limits the usable bandwidth. Experiments on principal modes in multimode fibers have demonstrated that the principal modes have a lower spatial dispersion than random wavefronts $[5,6,22]$. This effect is exploited in the generalized Wigner-Smith operator, a measurement of which allows operations such as focusing inside disordered media [17] and optical micromanipulation inside disordered media [23]. In this section we look at how the transmitted field of a time delay eigenstate disperses when changing the incident wavelength.

To measure the similarity as a function of wavelength detuning in the propagated field we define the similarity between two vectors $C S(\boldsymbol{a}, \boldsymbol{b})$,

$$
C S(\boldsymbol{a}, \boldsymbol{b})=\frac{|\boldsymbol{a} \cdot \boldsymbol{b}|}{\sqrt{|\boldsymbol{a}|^{2}} \sqrt{|\boldsymbol{b}|^{2}}}
$$

where $|\boldsymbol{a} \cdot \boldsymbol{b}|$ denotes the norm of the inner product between $\boldsymbol{a}$ and $\boldsymbol{b}$, and $|\boldsymbol{a}|^{2}$ the square of the norm of $\boldsymbol{a}$. We calculate the transmitted field by multiplying the eigenvectors $\boldsymbol{q}^{(n)}$ with the transmission matrix at a different 


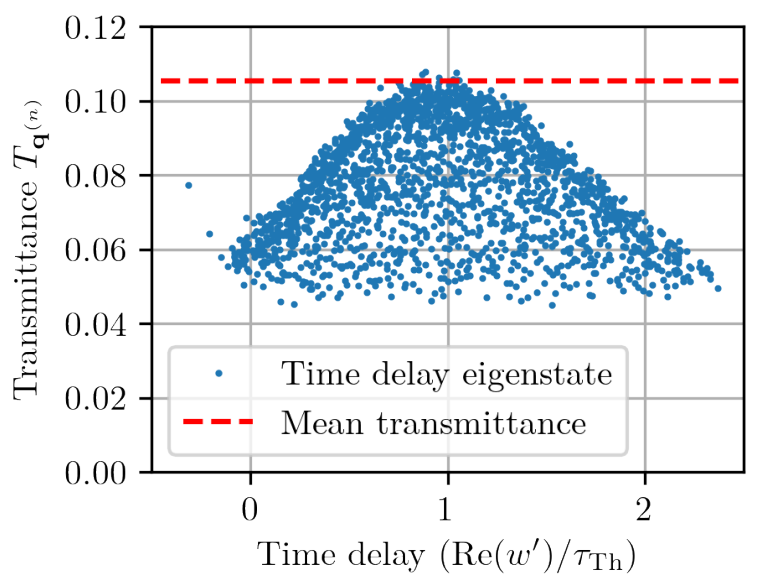

Figure 6.8.: Transmittance $T_{\boldsymbol{q}^{(n)}}$ versus time delay $\operatorname{Re}\left(w^{\prime}\right) / \tau_{\text {Th }}$ for time delay eigenstates on a $2.7 \mu \mathrm{m}$ sample. The average transmittance of the corresponding transmission matrix $\bar{T}$ is plotted in red. For the calculation of the eigenstates a cut off $\eta=0.07$ was used. For the transmission matrix $\bar{T}$ we find a maximum singular value $\sigma=0.8$.

wavelength $T(\Delta \lambda)$. Since we wish to compare the decorrelation of the time delay eigenstates with the speckle correlation function, we define a correlation function that is the square of the cosine similarity,

$$
C_{\boldsymbol{q}^{(n)}, \lambda_{0}}^{(n)}(\Delta \lambda)=C S\left(\bar{T} \boldsymbol{q}^{(n)}, T(\Delta \lambda) \boldsymbol{q}^{(n)}\right)^{2} .
$$

We calculate the correlation from our measured transmission matrices which give us access to detunings of $0.5 \mathrm{~nm}, 1.5 \mathrm{~nm}$, up to $4.5 \mathrm{~nm}$. For reference we also plot the measured speckle correlation function $C^{(1)}(\Delta \lambda)$ correlation values for the corresponding detuning values.

In Fig. 6.9, we plot the correlations $C_{\boldsymbol{q}^{(n)}, \lambda_{0}}^{(n)}(\Delta \lambda)$ versus the time delay eigenvalue for all detunings. We also plot the $C^{(1)}(\Delta \lambda)$ correlation values of the sample corresponding to the same detuning. We find that there is a large spread in correlation values $C_{\boldsymbol{q}^{(n)}, \lambda_{0}}^{(n)}(\Delta \lambda)$, with multiple values per time delay. We observe that the correlation values lie below the speckle correlation function in almost all cases. We find $C_{\boldsymbol{q}^{(n)}, \lambda_{0}}^{(n)}(\Delta \lambda=1.5 \mathrm{~nm})>$ $C^{(1)}(1.5 \mathrm{~nm})$ for 148 out of 1933 eigenstates and $C_{\boldsymbol{q}^{(n)}, \lambda_{0}}^{(n)}(\Delta \lambda=2.5 \mathrm{~nm})>$ 


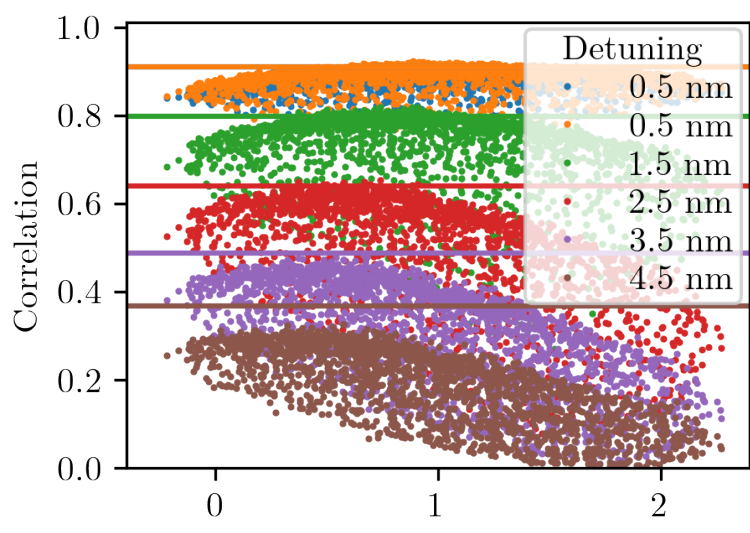

Time delay eigenvalue $\operatorname{Re}\left(\mathrm{w}^{\prime}\right) / \tau_{\mathrm{Th}}$

Figure 6.9.: Correlation $C_{\boldsymbol{q}^{(n)}, \lambda_{0}}^{(n)}(\Delta \lambda)$ (dots) versus time delay eigenvalue $\operatorname{Re}\left(w^{\prime}\right) / \tau_{\text {Th }}$ for different detuning values $\Delta \lambda$. The $C^{(1)}(\Delta \lambda)$ correlation values are plotted as a line for the corresponding detuning. Data calculated from $2.7 \mu \mathrm{m}$ sample.

$C^{(1)}(2.5 \mathrm{~nm})$ for 15 out of 1933 eigenstates. This clearly shows that the time delay eigenstates decorrelate faster than the $C^{(1)}(\Delta \lambda)$ correlation function. Additionally there is an asymmetry in the correlation between time delay eigenstates with positive and negative time delay. Eigenstates with a higher time delay decorrelate slightly faster. This might be due to the fact that these states interact more with the scattering medium.

\subsection{Conclusions}

In this chapter, we have presented measurements of the optical time delay eigenstates of a multiple scattering medium. We develop a method to find the time delay eigenstates of the scattering medium based on the reduced time delay operator and to measure the time delay of the eigenstates. We demonstrate that the measured time delay depends on the measurement method, however the distribution is consistent between the different methods except for a constant factor. All time delay eigenstates that we analyzed have a transmittance that is lower than the average transmittance. Finally 
6. Measurements of time delay eigenstates of light transmitted through scattering media

we demonstrated that the time delay eigenstates typically decorrelate faster than the speckle correlation function. As a future prospect, by measuring the reflection matrices in addition to the transmission matrices we envisage measuring a much larger part of the full time-delay operator which should have a wider range of real eigenvalues. 


\section{Bibliography}

[1] E. P. Wigner, "Lower limit for the energy derivative of the scattering phase shift," Phys. Rev. 98, 145-147 (1955).

[2] F. T. Smith, "Lifetime matrix in collision theory," Phys. Rev. 118, 349-356 (1960).

[3] B. Gérardin, J. Laurent, P. Ambichl, C. Prada, S. Rotter, and A. Aubry, "Particlelike wave packets in complex scattering systems," Phys. Rev. B 94, 014209 (2016).

[4] J. Böhm, A. Brandstötter, P. Ambichl, S. Rotter, and U. Kuhl, "In situ realization of particlelike scattering states in a microwave cavity," Phys. Rev. A 97, 021801 (2018).

[5] W. Xiong, P. Ambichl, Y. Bromberg, B. Redding, S. Rotter, and H. Cao, "Spatiotemporal control of light transmission through a multimode fiber with strong mode coupling," Phys. Rev. Lett. 117, 053901 (2016).

[6] W. Xiong, P. Ambichl, Y. Bromberg, B. Redding, S. Rotter, and H. Cao, "Principal modes in multimode fibers: exploring the crossover from weak to strong mode coupling," Opt. Express 25, 2709-2724 (2017).

[7] A. Brandstötter, A. Girschik, P. Ambichl, and S. Rotter, "Shaping the branched flow of light through disordered media," Proc. Natl. Acad. Sci. USA 116, 13260 (2019).

[8] M. Durand, S. M. Popoff, R. Carminati, and A. Goetschy, "Optimizing light storage in scattering media with the dwell-time operator," Phys. Rev. Lett. 123, 243901 (2019).

[9] O. Katz, E. Small, Y. Bromberg, and Y. Silberberg, "Focusing and compression of ultrashort pulses through scattering media," Nat. Photonics 5, 372-377 (2011).

[10] J. Aulbach, B. Gjonaj, P. M. Johnson, A. P. Mosk, and A. Lagendijk, "Control of light transmission through opaque scattering media in space and time," Phys. Rev. Lett. 106, 103901:1 -4 (2011).

[11] Y. Choi, T. R. Hillman, W. Choi, N. Lue, R. R. Dasari, P. T. C. So, W. Choi, and Z. Yaqoob, "Measurement of the time-resolved reflection matrix for enhancing light energy delivery into a scattering medium," Phys. Rev. Lett. 111, 243901 (2013).

[12] S. Kang, S. Jeong, W. Choi, H. Ko, T. D. Yang, J. H. Joo, J.-S. Lee, Y.-S. Lim, Q.-H. Park, and W. Choi, "Imaging deep within a scattering 
medium using collective accumulation of single-scattered waves," Nat. Photonics 9, 253 (2015).

[13] M. Mounaix, D. Andreoli, H. Defienne, G. Volpe, O. Katz, S. Grésillon, and S. Gigan, "Spatiotemporal coherent control of light through a multiple scattering medium with the multispectral transmission matrix," Phys. Rev. Lett. 116, 253901 (2016).

[14] M. Mounaix, H. Defienne, and S. Gigan, "Deterministic light focusing in space and time through multiple scattering media with a time-resolved transmission matrix approach," Phys. Rev. A 94, 041802 (2016).

[15] M. Mounaix, H. B. de Aguiar, and S. Gigan, "Temporal recompression through a scattering medium via a broadband transmission matrix," Optica 4, 1289-1292 (2017).

[16] P. Ambichl, "Coherent wave transport: time-delay and beyond," $\mathrm{PhD}$ thesis, Technische Universität Wien, Vienna (2016).

[17] P. Ambichl, A. Brandstötter, J. Böhm, M. Kühmayer, U. Kuhl, and S. Rotter, "Focusing inside disordered media with the generalized wigner-smith operator," Phys. Rev. Lett. 119, 033903 (2017).

[18] L. Eisenbud, "The Formal Properties of Nuclear Collisions." PhD thesis, Princeton University (1948).

[19] R. Landauer and M. Buttiker, "Diffusive traversal time: Effective area in magnetically induced interference," Phys. Rev. B 36, 6255-6260 (1987).

[20] I. M. Vellekoop, P. Lodahl, and A. Lagendijk, "Determination of the diffusion constant using phase-sensitive measurements," Phys. Rev. E 71, 056604 (2005).

[21] S. Rotter and S. Gigan, "Light fields in complex media: Mesoscopic scattering meets wave control," Rev. Mod. Phys. 89, 015005 (2017).

[22] J. Carpenter, B. J. Eggleton, and J. Schröder, "Observation of eisenbudwigner-smith states as principal modes in multimode fibre," Nat. Photonics 9, 751 (2015).

[23] M. Horodynski, M. Kühmayer, A. Brandstötter, K. Pichler, Y. Fyodorov, U. Kuhl, and S. Rotter, "Optimal light fields for micromanipulation in complex scattering environments," in "Frontiers in Optics + Laser Science APS/DLS," (Optical Society of America, 2019), p. FW6B.3. 


\section{Scattering invariant modes ${ }^{1}$}

\subsection{Introduction}

Some special field superpositions in multiple scattering media exhibit peculiar and useful properties. Among others, it has been shown that it is possible to create one or multiple foci through scattering materials $[1,2]$, increase transmission [3-7], and transport quantum states in a determinate way $[8]$.

Another special field are the scattering invariant modes (SIMs), which are fields that are transmitted the same through a multiply scattering medium and a layer of air except for a global phase and amplitude difference. This means that for these modes it is impossible to determine if the scattering medium is present or not from only measuring the transmitted field. In Fig. 7.1 the concept of SIMs is depicted: the same field is transmitted through both a scattering medium and a clear piece of air. Mathematically the scattering invariant modes can be described by the generalized eigenvalue problem

$$
T_{\mathrm{s}} \tilde{\boldsymbol{E}}_{\mathrm{n}}=\alpha_{n} T_{\mathrm{air}} \tilde{\boldsymbol{E}}_{\mathrm{n}},
$$

where $\alpha_{n}$ corresponds to the complex eigenvalue, $\tilde{\boldsymbol{E}}_{\mathrm{n}}$ to a generalized eigenvector and $T_{\mathrm{s}}$ and $T_{\text {air }}$ to the transmission matrices of a sample and air respectively.

In this chapter, we describe a method to find scattering invariant modes. We look at different methods for solving and finding an approximate solution to the generalized eigenvalue problem, and look at experimental results of projected and calculated scattering invariant modes. Finally we perform wavefront shaping with scattering invariant modes, and introduce the concept of sparse scattering invariant modes.

\footnotetext{
${ }^{1}$ The results of this chapter will be part of a forthcoming publication: P. Pai, J. Bosch, M. Kühmayer, S. Rotter and A. P. Mosk (in preparation)
} 


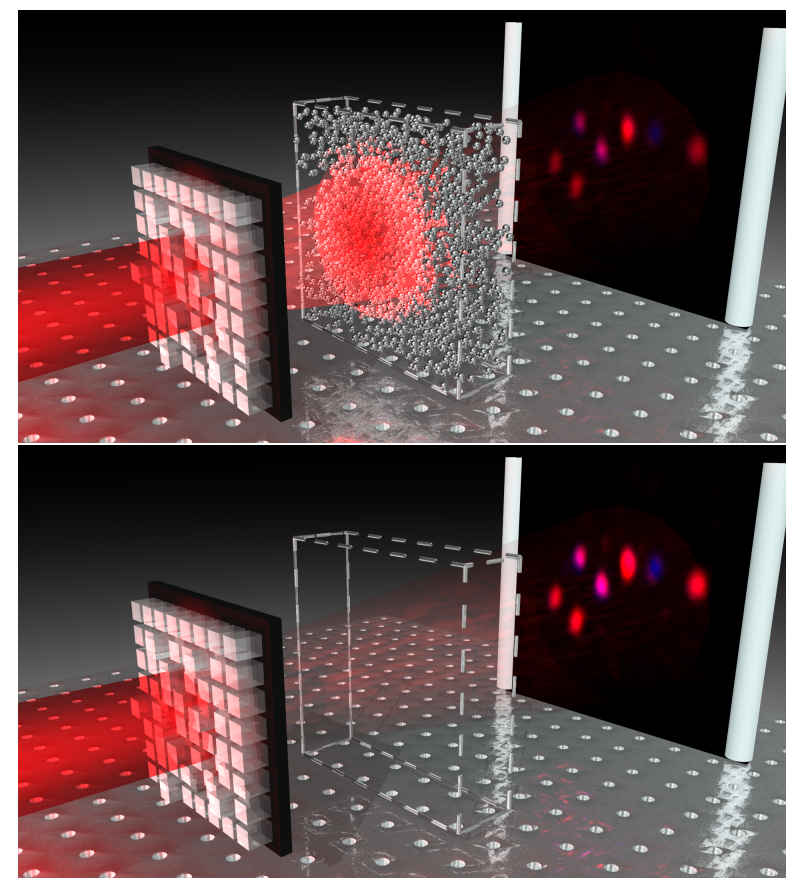

Figure 7.1.: Cartoon of a SIM $\tilde{\boldsymbol{E}}_{\mathrm{n}}$ propagating through a scattering layer (top) and an air layer (bottom). The same field is transmitted through both materials, except for a global amplitude factor $\alpha[9]$.

\subsection{Finding scattering invariant modes}

The scattering invariant modes can be found in different ways. The easiest method for finding the scattering invariant modes is by solving equation (7.1) for two measured transmission matrices. We calculate the scattering invariant modes based on a matrix inversion. The numerical inverse is unstable due to of measurement noise and low singular values in the transmission matrix. Therefore we implement two different matrix inversion methods. For the first method, from Eq. 7.1 we multiply the left hand size and the right hand side with the inverse of the transmission matrix of air,

$$
T_{\mathrm{air}}^{-1} T_{\mathrm{s}} \tilde{\boldsymbol{E}}_{\mathrm{n}}=\alpha_{n} \tilde{\boldsymbol{E}}_{\mathrm{n}}
$$


We solve this eigenvalue problem by calculating the eigenvectors of $T_{\text {air }}^{-1} T_{\mathrm{s}}$ with a numeric eigenvalue solver. One major disadvantage of this method is that it relies on inverting $T_{\text {air }}$, which is unstable due to singular values close to zero $[10,11]$. This introduces spurious high eigenvalues with non-physical amplitudes $\left|\alpha_{n}\right|>1$, which correspond to states with a higher transmittance through the scattering layer than through the air.

The second method for calculating the scattering invariant modes approximates the inverse of $T_{\text {air }}$ with its Hermitian transpose. This approximation is not sensitive to low singular values in the quasi-inversion. The air transmission matrix $T_{\text {air }}$ is close to unitary, which makes this a valid approximation such that we can calculate scattering invariant modes which propagate through the two samples with a high similarity. Hence Eq. 7.1 can be approximated as

$$
T_{\text {air }}^{\dagger} T_{\mathrm{s}} \tilde{\boldsymbol{E}}_{\mathrm{n}}=\alpha_{n} \tilde{\boldsymbol{E}}_{\mathrm{n}}
$$

We calculate the eigenvectors from Eq. (7.3) in the same way as from Eq. (7.2).

The transmission matrices are measured according to the measurement procedure outlined in Chapter 2.7. To reiterate, we project the incident fields with a high NA objective (0.95NA) and sample the transmitted fields with another high NA objective (1.4NA, oil immersion) over a large field of view with a radius of $23 \mu \mathrm{m}$. We sample the transmission matrix at 2282 points on a hexagonal grid in $k$-space [12], sampling the field up to an NA of approximately 0.9 on both sides.

In Fig. 7.2, we plot (a) the eigenvalue spectrum of a 2.1-um-thick scattering sample, calculated from $T_{\text {air }}^{\dagger} T_{\mathrm{s}}$ and (b) the eigenvalue spectrum calculated from $T_{\text {air }}^{-1} T_{\mathrm{s}}$. For the eigenvalues calculated with the Hermitian transpose, we find a drop in density along the radial direction no angular dependence of the density. For the eigenvalues calculated from the inverse we find an eigenvalue density that is independent of the amplitude of $\alpha$ or the angle. This indicates that there is a difference between the SIMs calculated from our two methods.

The propagated fields show a visual similarity between the field propagated through air (Fig. $7.3(\mathrm{~b}, \mathrm{~d})$ ) and through the scattering medium (Fig. $7.3(\mathrm{a}, \mathrm{c})$ ). To quantify how well the SIMs are approximated with our two calculation methods, we use the cosine similarity of the fields propagated through the scattering medium. We define the cosine similarity between the 


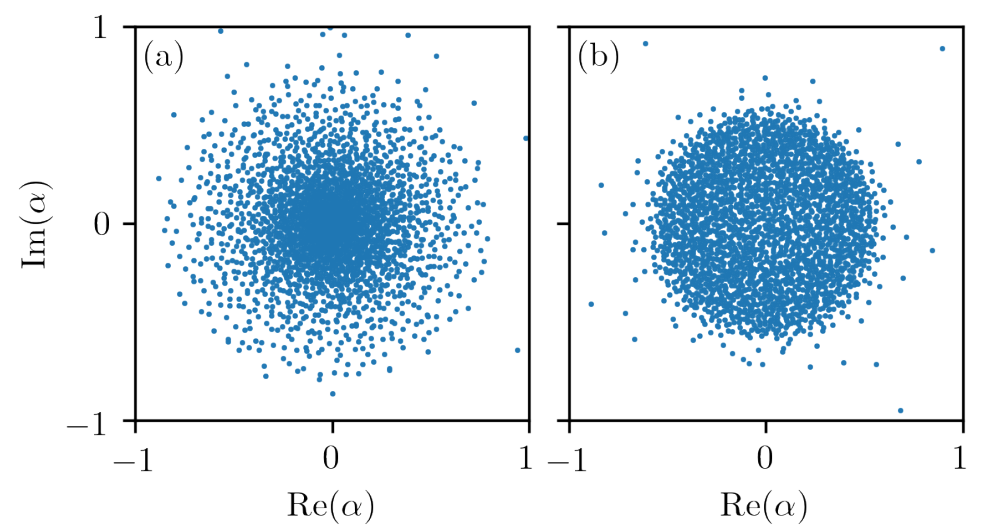

Figure 7.2.: Eigenvalue spectrum of (a) $T_{\text {air }}^{\dagger} T_{\mathrm{s}}$ and (b) $T_{\text {air }}^{-1} T_{\mathrm{s}}$. Measured on a 2.1- $\mu \mathrm{m}$-thick sample.

vectors $\boldsymbol{a}$ and $\boldsymbol{b}$ according to

$$
C S(\boldsymbol{a}, \boldsymbol{b})=\frac{|\boldsymbol{a} \cdot \boldsymbol{b}|}{\sqrt{|\boldsymbol{a}|^{2}} \sqrt{|\boldsymbol{b}|^{2}}} .
$$

A cosine similarity of 1 corresponds to the same propagated field, with a possible difference in global phase and amplitude, and a cosine similarity of 0 corresponds to an orthogonal field. We now calculate the cosine similarity of the numerically propagated field: $C S\left(T_{\text {air }} \tilde{\boldsymbol{E}}_{\mathrm{n}}, T_{\mathrm{s}} \tilde{\boldsymbol{E}}_{\mathrm{n}}\right)$, where we calculated the propagated field by multiplication with the respective transmission matrix. We also physically propagate the SIMs through both the air and the scattering medium, and calculate the cosine similarity from the propagated fields: $C S\left(\tilde{\boldsymbol{E}}_{\text {air }, \mathrm{n}, t}, \tilde{\boldsymbol{E}}_{s, \mathrm{n}, t}\right)$, where $\tilde{\boldsymbol{E}}_{\text {air,n,t }}$ indicates the field physically propagated through air, sampled on the same grid as the transmission matrix. In the experiment, we first measure the transmission matrices and calculate the corresponding holograms for the SIMs. Then we project all the SIMs on the air layer position and finally project the SIMs on the scattering medium. We project the full SIMs, with both the horizontal and vertical polarization component and control both phase and amplitude of the fields. For every projected SIM we measure the full transmitted field.

In Fig. 7.3 (e), we plot the cosine similarity for three methods of finding and projecting the SIMs. We first discuss the cosine similarity between the 

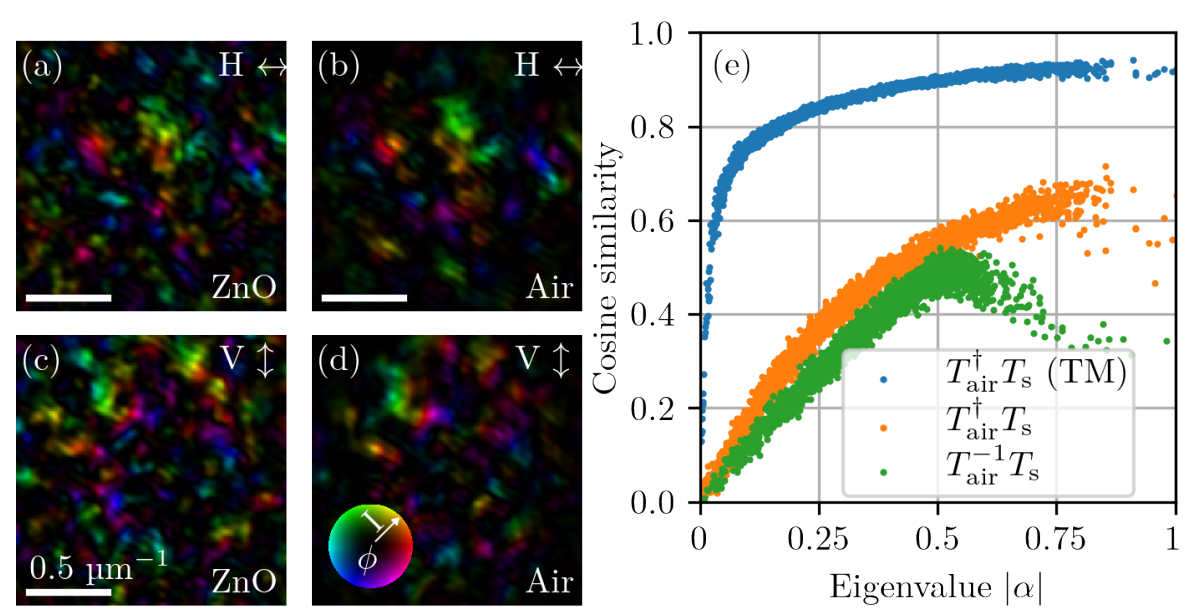

Figure 7.3.: Transmitted field of a SIM (a-d) with a cosine similarity of 0.68. In (e) the measured cosine similarity for the propagated SIMs of $T_{\text {air }}^{\dagger} T_{\mathrm{s}}$ through the transmission matrices (blue), or physically through the sample (orange) and the eigenvectors of $T_{\text {air }}^{-1} T_{\mathrm{s}}$ propagated through the sample (green). The cosine similarity has been calculated from the sampled fields.

vectors calculated from $T_{\text {air }}^{\dagger} T_{\mathrm{s}}$, physically propagated through the two materials. We find that the cosine similarity increases with the SIM eigenvalue, and 775 out of 2282 propagated eigenvectors have a cosine similarity above 0.5 , with a maximum of 0.72 . In green we plot the cosine similarity for the eigenvectors calculated from $T_{\text {air }}^{-1} T_{\mathrm{s}}$, physically propagated through the scattering material and air. We find that the cosine similarity is generally lower than for the projected vectors obtained from the Hermitian conjugate, a maximum cosine similarity of 0.54 , and 157 eigenvectors with a cosine similarity above 0.5. All cosine similarity are calculated from the sampled field vectors, which is the same field as described by the transmission matrix. For the field depicted in Fig. 7.3 (a-d) we find a cosine similarity of 0.68. We found a higher cosine similarity in the propagated field for SIMs calculated with the Hermitian conjugate indicating that this method produced the best experimental approximation. Therefore we use this method in the following sections. 
For the case of SIMs numerically propagated through the transmission matrix, the cosine similarity shows a different behavior. For the SIMs calculated using matrix inversion, the cosine similarity is by definition unity for all modes (not plotted), while for the SIMs calculated using Hermitian conjugation the cosine similarity increases with the eigenvalue and quickly reaches a high value of about 0.9 . In all cases the cosine similarity as found from numerical propagation exceeds the one found in the experiment. This is due to a combination of limited fidelity of the wavefront synthesizer and an overestimation of the similarity in the numerical propagation since the noise in the measurement correlates with the noise in the digital propagation.

\subsection{Superpositions of scattering invariant modes}

The scattering invariant modes are speckle-like fields. By creating superpositions of SIMs it is possible to project simple images. If the SIMs have similar eigenvalues $\alpha$, these superpositions are propagated the same through the scattering medium and air, and the same image will be projected. As a proof of concept we optimize the transmitted intensity of the superposition in a single point.

To create the superpositions we choose two different groups of SIMs, which are indicated in Fig. 7.4. For the first group (a) we select a set of eigenvalues with similar phase and amplitude. We select a set of modes with eigenvalues in a small radius $r_{2}$ around value $r_{1}$ at an angle $\phi$ in the complex plane. By choosing suitable values this gives us a set of eigenvalues with similar phase and amplitude. For the second group (b) we select a set of modes with similar amplitude, chosen withing a radius range from the origin. We choose the input field as a sum of the corresponding scattering invariant modes with optimized relative phase and amplitude of the modes. For this we initialize the input field as a linear sum of the set of eigenvectors. Next we vary the phase and amplitude of the modes: for each eigenvector we propagate the input field with the transmission matrix for different phases. We set the phase corresponding to the highest field sum in the optimization points. The amplitude of the mode we set equal to the visibility of modulating the specific mode. We note this method is similar to the method by Vellekoop [1].

We project the superpositions through both the air and the scattering 

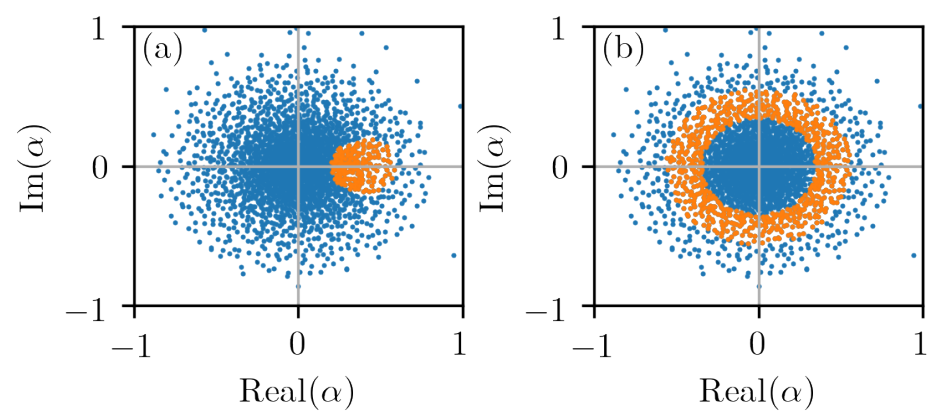

Figure 7.4.: Eigenvalues of SIMs used in superpositions. The eigenvalues in blue, the SIMs in the superposition indicated in orange. In (a) we select eigenvalues with similar phase and amplitude, in (b) we select eigenvalues with similar amplitude only.

medium. We image the transmitted intensity on a single camera, on which both polarization components are combined. In Fig. 7.5 we plot the intensity pattern of the transmitted light where we have increase the intensity in a single focus. In (a) and (c) we select modes with eigenvalues with similar phase and amplitude, and in (b) and (d) we select modes with only a similar amplitude. In order to increase the contrast we integrate the image over 6 different superpositions by setting a long exposure time on the camera, and projecting the different fields. We optimize for a focus through the scattering medium. As expected, we find that for the superposition consisting of eigenvectors with eigenvalues with similar phase, we get a visible focus through both the scattering medium and through air, while by selecting on eigenvalues with similar amplitude, we only project a focus through the material for which the optimization was performed.

We achieve an enhancement contrast of about 3 (Fig. 7.5 (a)), measured in focus intensity over average background intensity. A focus with this contrast is similar to a bright random speckles, as seen in (Fig. $7.5(\mathrm{~d})$ ). The enhancement reached by this procedure depends on the location on the sample, and is sometimes too low for the focus to be discernible. This is due to the fact that we have a limited set of independent basis vectors for the superposition and at some positions even their full constructive interference may not give rise to a high amplitude. 


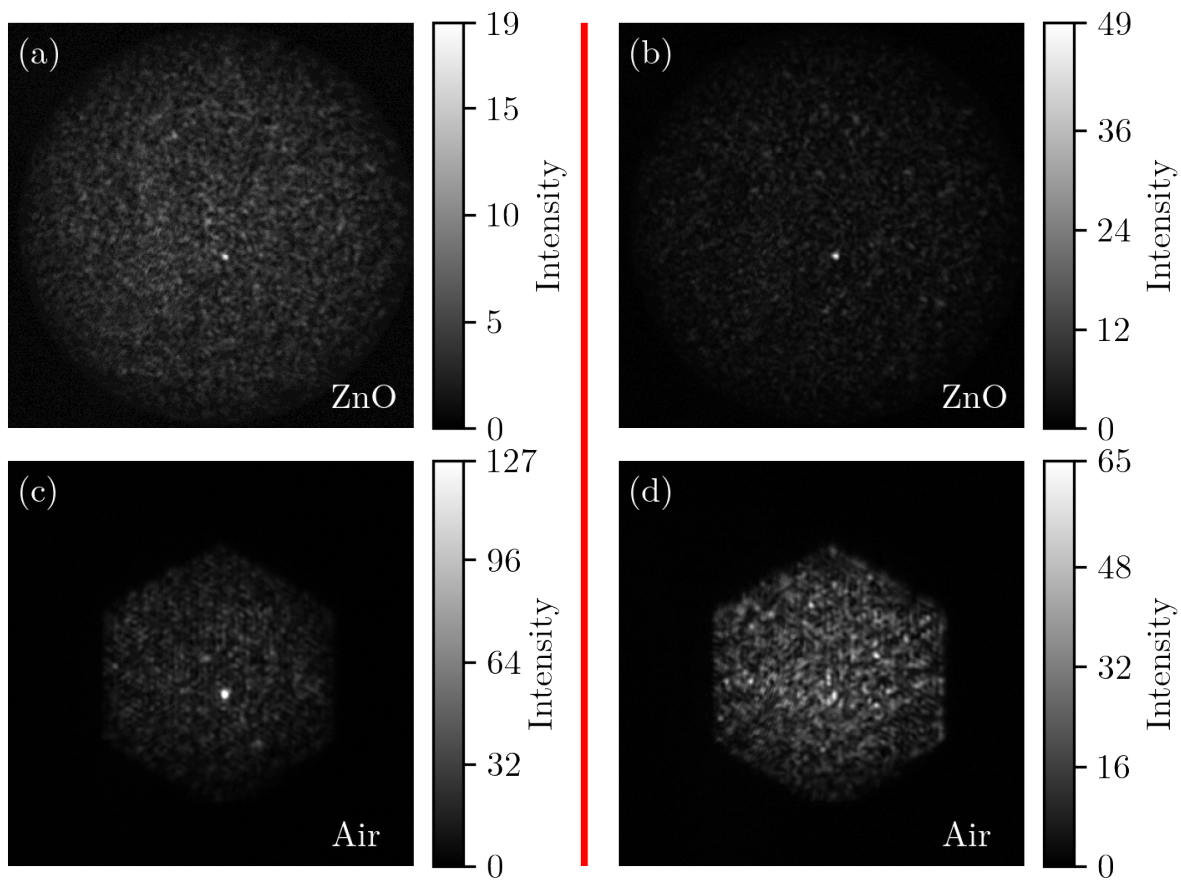

Figure 7.5.: Transmitted intensity through the scatterer (a,b) and through air (c,d). The left column (a,c) corresponds to a focus shaped with scattering invariant modes with eigenvalues with similar phase, the right column (b,d) corresponds to a focus shaped with scattering invariant modes with eigenvalues with similar amplitude. Measurements performed on a 2.1-um-thick sample.

Although we have successfully projected a focus through two mediums at the same time using this method, the low enhancement is a key limitation.

\subsection{Sparse scattering invariant modes}

When projecting simple patterns or images we are only interested in a limited number $M$ of the transmitted modes, where $\mathrm{M}$ is much smaller than the total number of transmitted modes $N$. For these situations we define the sparse scattering invariant modes (SSIMs). We define a SSIMs as a vectors $\boldsymbol{p}_{n}$ for 
which: $\tilde{T}_{\mathrm{s}} \boldsymbol{s}=\boldsymbol{p}$ and $\tilde{T}_{\text {air }} \boldsymbol{s}=\alpha \boldsymbol{p}$, where $\tilde{T}_{\mathrm{s}}$ and $\tilde{T}_{\text {air }}$ are the submatrices of $T_{\mathrm{s}}$ and $T_{\text {air }}$ that contain only the rows corresponding to the $M$ transmitted modes of interest, ignoring the $(N-M)$ other modes.

We create a sparse scattering invariant mode by numerically conjugating the target field through both transmission matrices and coherently add the two resulting vectors. If we set $\boldsymbol{p}$ as he goal field, we approximate the incident field on the scattering sample as $\approx T_{\mathrm{s}}^{\dagger} \boldsymbol{p}$ and the incident field on the air as $\approx T_{\text {air }}^{\dagger} \boldsymbol{p}$. We construct the scattering invariant mode as a superposition of these two fields: $s=T_{\mathrm{s}}^{\dagger} \boldsymbol{p}+T_{\text {air }}^{\dagger} \boldsymbol{p}$. In Fig. 7.6 we plot an experimentally projected sparse scattering invariant mode, that forms an independent seven point constellation in each polarization component. In (a) and (b) we see the horizontal polarization component shaped as Ursa minor and propagated through $\mathrm{ZnO}$ and air respectively. In (c) and (d) we see the horizontal polarization component that is shaped as Ursa major through $\mathrm{ZnO}$ and air respectively. The phase and amplitude in each spot can be set individually. The same hologram is projected through both mediums, and we project the phase and amplitude for both input polarizations of the hologram simultaneously. We find a high contrast between the constellation and an easily recognizable shape. 

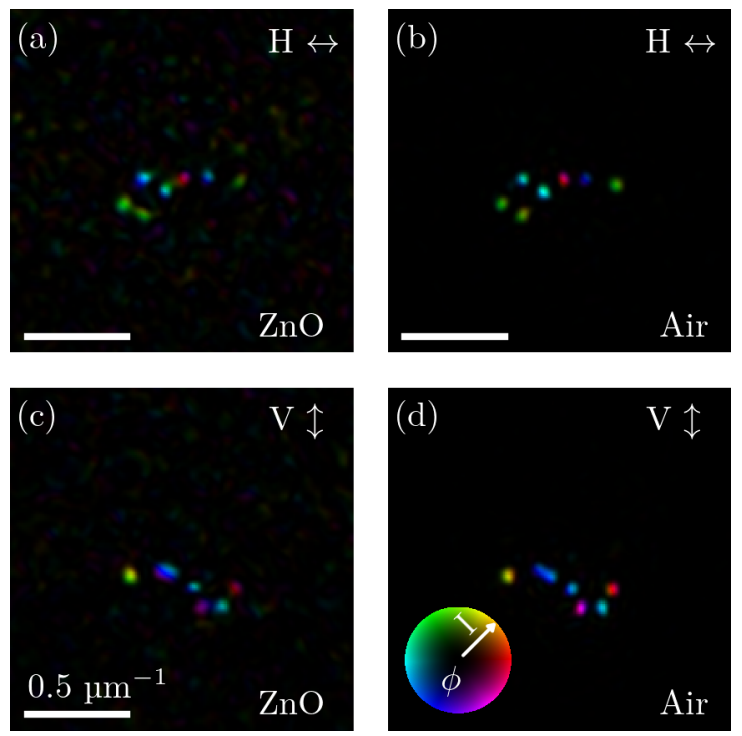

Figure 7.6.: Experimentally generated sparse scattering invariant mode. Propagated through a 5.2- $\mu$ m-thick $\mathrm{ZnO}$ sample (a,c) and a 5.2 - $\mu$ m-thick layer of air $(b, d)$. The color scale encodes both phase and amplitude. The same incident field is projected through both the $\mathrm{ZnO}$ and the air.

\subsection{Conclusion}

In this chapter, we have experimentally demonstrated scattering invariant modes. The scattering invariant modes are fields that have a high similarity propagated through a scattering medium and a layer of air except for a global phase and amplitude difference. We have presented two different methods for finding scattering invariant modes. We found that by approximating the inverse of the transmission matrix with its complex conjugate instead of the numerical inverse, we get scattering invariant modes with a higher cosine similarity between the field propagated through the different materials. From the scattering invariant modes we find a high cosine similarity between the field propagated through air and a zinc oxide scattering medium of up to 0.72 . We have demonstrated that superpositions of scattering invariant modes with similar phase of the eigenvalue create a field that projects a simple image 
when propagated through either of two different materials. Finally we have experimentally demonstrated sparse scattering invariant modes, which are scattering invariant modes that create a sparse transmitted pattern.

These results demonstrate that we generate scattering invariant modes with a high cosine similarity. We have found that calculating the SIMs from $T_{\text {air }}^{\dagger} T_{\mathrm{s}}$ allows us to find SIMs that have higher eigenvalues and cosine similarities after propagation through the sample and reference. The methods of elucidation and control of SIMs are a basis to investigate the properties of the scattering invariant modes, such as the interactions with the scattering medium and their relationship to ballistic waves. 


\section{Bibliography}

[1] I. M. Vellekoop and A. P. Mosk, "Focusing coherent light through opaque strongly scattering media," Opt. Lett. 32, 2309-2311 (2007).

[2] S. Popoff, G. Lerosey, R. Carminati, M. Fink, A. Boccara, and S. Gigan, "Measuring the transmission matrix in optics: An approach to the study and control of light propagation in disordered media," Phys. Rev. Lett. 104, 100601 (2010).

[3] I. M. Vellekoop and A. P. Mosk, "Universal optimal transmission of light through disordered materials," Phys. Rev. Lett. 101, 120601 (2008).

[4] S. M. Popoff, A. Goetschy, S. Liew, A. Stone, and H. Cao, "Coherent control of total transmission of light through disordered media," Phys. Rev. Lett. 112, 133903 (2014).

[5] M. Kim, Y. Choi, C. Yoon, W. Choi, J. Kim, Q.-H. Park, and W. Choi, "Maximal energy transport through disordered media with the implementation of transmission eigenchannels," Nat. Photonics 6, 581-585 (2012).

[6] X. Hao, L. Martin-Rouault, and M. Cui, "A self-adaptive method for creating high efficiency communication channels through random scattering media," Sci. Rep. 4, 5874 (2014).

[7] J. Bosch, S. A. Goorden, and A. P. Mosk, "Frequency width of open channels in multiple scattering media," Opt. Express 24, 26472-26478 (2016).

[8] T. J. Huisman, S. R. Huisman, A. P. Mosk, and P. W. H. Pinkse, "Controlling single-photon fock-state propagation through opaque scattering media," Appl. Phys. B 116, 603-607 (2014).

[9] P. Pai, J. Bosch, M. Kühmayer, S. Rotter, and A. P. Mosk (in preparation).

[10] M. Nieto-Vesperinas, Scattering and Diffraction in Physical Optics, 2nd Edition (World Scientific Publishing Company, 2006).

[11] M. Tanter, J.-L. Thomas, and M. Fink, "Time reversal and the inverse filter," J. Acoust. Soc. Am. 108, 223-234 (2000).

[12] P. Pai, J. Bosch, and A. P. Mosk, "Optical transmission matrix measurement sampled on a dense hexagonal lattice," OSA Continuum 3, 637-648 (2020). 


\section{Iterative algorithm for finding scattering invariant modes}

\subsection{Introduction}

Highly transmitting transmission channels can be found through iterative phase conjugation $[1,2]$, without requiring transmission matrix measurements. These highly transmitting channels are the eigenvectors of the matrix $T_{\mathrm{s}}^{\dagger} T_{\mathrm{s}}$. The phase conjugation procedure has an interesting similarity with Andreev reections in a superconductor-metal junction [3-5]. We show the implementation of a a modified procedure, to find a scattering invariant mode of a system, which is the largest eigenvector of the matrix $T_{\text {air }}^{\dagger} T_{\mathrm{s}}$ as demonstrated in Chapter 7. Our method is similar to the von Mises iteration in numerical algebra. This iteration is known to converge slowly if there are many eigenvalues with equal modulus [6]. In our physical implementation for solving the generalized eigenvalue equation, we use a symmetry breaking method to provide a robust and quickly converging solution. In this chapter, we present simulation results for different approaches based on transmission matrix measurements and experimental results of the iterative digital phase conjugation procedure.

\subsection{Methods}

We demonstrate an experimental method for finding scattering invariant modes based on iterative phase conjugation. In iterative phase conjugation, light is alternately phase conjugated through two mediums. Here the op-

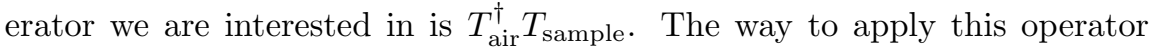
repeatedly is by first projecting a random generated field through the sample and measure and store the transmitted field. The projection is turned 
off, and the the phase conjugate of the transmitted field is projected in the opposite direction through the air medium. Again we this transmitted field is measured and stored, and subsequently the phase conjugate field is projected through the scattering material. The idea underlying this method is the von Mises iteration [7], which is a power iteration that yields the eigenvector of an operator corresponding to the largest eigenvalue by repeated application of the operator. In this section we describe the measurement procedure and the different implementations that we have investigated.

The procedure for finding a SIM of a scattering medium and air layer is illustrated in Fig. 8.1. This experimental procedure is as follows. We start by projecting a random field $\left(\boldsymbol{E}_{0}\right)$ from $\mathrm{S} 1$ trough the scattering medium and measure the transmitted field $\left(\boldsymbol{E}_{1}\right)$ on D2. We store the field in memory and turn off the projection. Next, we shift the substrate and refocus on the air medium, which is a cleaned part of the substrate. We send the conjugate field $\left(\boldsymbol{E}_{1}^{*}\right)$ back through the air on S2 and measure and save the transmitted field $\left(\boldsymbol{E}_{2}\right)$ on D1. We turn off the projection from S2 and shift back to the scattering position, refocus the objectives, and send the conjugate field $\left(\boldsymbol{E}_{2}^{*}\right)$ from S1 through the scattering material. The transmitted field $\left(\boldsymbol{E}_{3}\right)$ is measured on D2 and stored in memory. Next, we add a symmetry breaking term which is either a fraction of the field from the previous round $\boldsymbol{E}_{1}$, or a constant field $\boldsymbol{E}_{\mathrm{Off}}$, to this field as an offset with mixing parameter $\beta$ : $\left(\boldsymbol{E}_{3}^{*}+\beta \boldsymbol{E}_{1}^{*}\right)$. We project this field back through the air and measure the transmitted field $\left(E_{4}\right)$. We keep iterating this loop, where we add the symmetry breaking term when propagating through the air, but not on the step where we propagate through the scattering material. To monitor convergence to a scattering invariant mode, we propagate every field through both materials (e.g. $\boldsymbol{E}_{0}$ is also transmitted through air) and record the transmitted field.

To calculate the convergence of the iterative phase conjugation we define the cosine similarity $C S(\boldsymbol{a}, \boldsymbol{b})$ as,

$$
C S(\boldsymbol{a}, \boldsymbol{b})=\frac{|\boldsymbol{a} \cdot \boldsymbol{b}|}{\sqrt{|\boldsymbol{a}|^{2}} \sqrt{|\boldsymbol{b}|^{2}}} .
$$

We calculate the cosine similarity of the fields propagated through the scattering sample and the air traversing in the same direction every round: $C S\left(\boldsymbol{E}_{n, \text { sample }}, \boldsymbol{E}_{n, \text { air }}\right)$. 


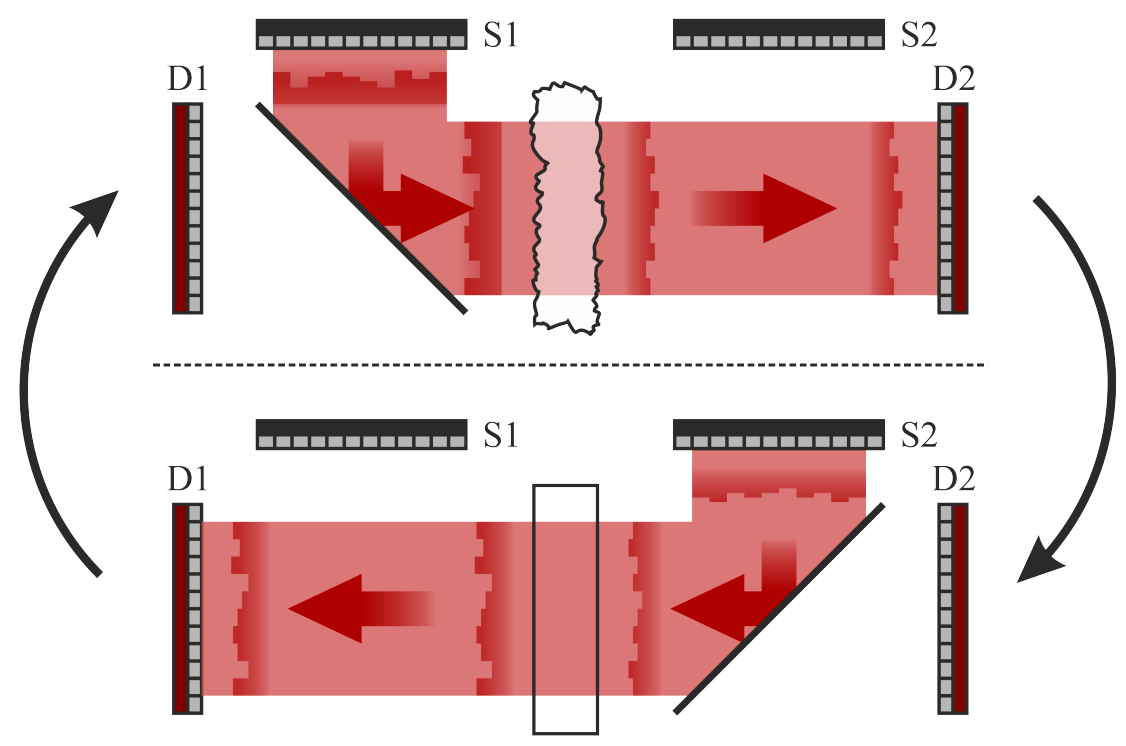

Figure 8.1.: Schematic procedure for finding scattering invariant mode with iterative phaseconjugation. Figure adapted from [8].

\subsection{Simulation of iterative algorithm for finding a SIM}

We have simulated the process of iterative phase conjugation to analyze convergence and solutions of the method. Our simulations are based on two measured transmission matices. The transmission matrices correspond to a measurement of a $\mathrm{ZnO}$ multiple scattering sample of $2.1 \mu \mathrm{m}$ thickness, the air transmission matrix corresponds to an air column of the same thickness. We simulate backwards propagation through the air with the conjugate transpose of the air transmission matrix because this corresponds best to our experimental procedure of phase conjugating. We initialize a random vector, and run through 21 iterations of phase conjugations, corresponding to a practical number of phase conjugations. Before every propagation step, we add random noise to the field, to simulate incomplete control and aberrations in the phase conjugation procedure. We run three different simulations where we simulate the case without symmetry breaking, with a constant 
symmetry breaking term and with a symmetry breaking term proportional to the field in the previous iteration. We initialize these simulations with the same random vector but apply uncorrelated random noise in every iteration. We calculate the cosine similarity between the field propagated through air and the scattering medium every iteration according to equation (8.1).

In Fig. 8.2, we plot simulation results for finding scattering invariant modes with iterative phase conjugation based on a set of measured transmission matrices. The transmission matrices are measured on the same sample, and with the same focussing conditions as the actual iterative phase conjugation measurement reported in this chapter. We plot the mean and standard deviation of the correlation over 100 independent runs. In Fig. 8.2 (a) we plot the results for digital iterative phase conjugation without symmetry breaking, in (b) we plot the results with a constant symmetry breaking term and in (c) we show the results where the previous iteration field is used as a symmetry breaking term. We plot the results for 3 different amounts of applied noise: $10 \%$ (blue), 30\% (orange) and 50\% (green). The amount of noise is measured relative to the total transmitted power. We find that an increase in noise leads to lower cosine similarities for all three methods. For the same amount of noise we find that no symmetry breaking term gives the lowest cosine similarity, and the largest variance between different runs. A constant symmetry breaking term gives higher cosine similarity and suppresses the noise. By using the previous iteration field as a symmetry breaking term, we find the highest cosine similarity, even in the presence of strong noise. We also find that we consistently find a very similar cosine similarity.

The improved convergence by applying a symmetry breaking term is explained by the fact that it adds a bias to the system. Similar to how iterative phase conjugation converges to the most open channel $[1,2]$, our iterative phase conjugation converges to the highest generalized eigenvalue. However, if the system has converged to a superposition of two SIMs with eigenvalues that have a $\pi$ difference in argument but the same amplitude, convergence will halt. The procedure will cycle through different superpositions of these two SIMs, which is not a SIM itself. By adding a bias to the system, SIMs with similar argument are favored, which avoids breakdown of convergence. 


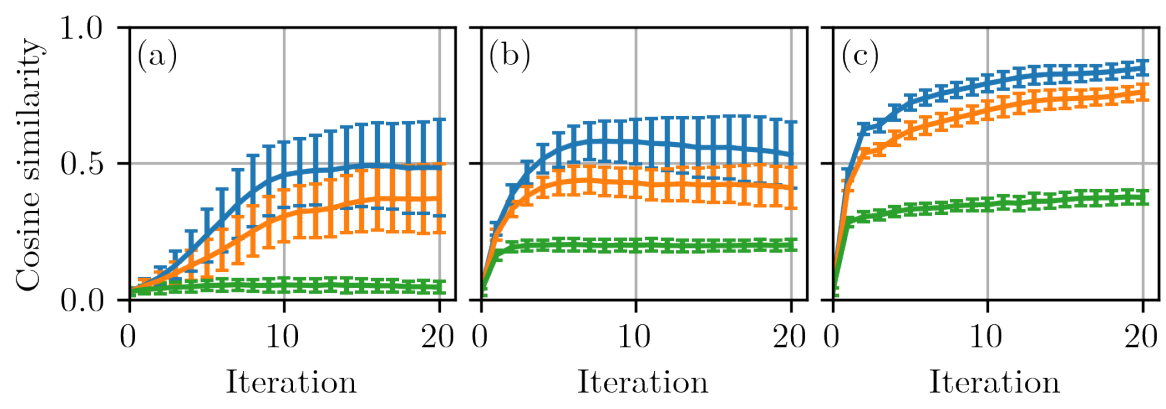

Figure 8.2.: Simulated iterative phase conjugation procedures. (a) Cosine similarity versus iteration without symmetry breaking, (b) with a constant symmetry breaking term, (c) with the previous iteration field as a symmetry breaking term. Blue: 10\% noise, relative to total power, orange, 30\%, green, 50\%. Errorbars: standard deviation between different runs.

\subsection{Finding SIM through digital optical phase conjugation}

We have experimentally implemented the iterative phase conjugation procedure with the same 2.1 - $\mu$ m-thick $\mathrm{ZnO}$ (mean free path estimated $l \approx 0.7 \mu \mathrm{m}$ [9]) sample used in Chapter 7. This allows us to make a comparison between the physical and simulated iterative phase conjugation measurement.

In Fig. 8.3 (a) we plot the cosine similarity between the fields propagated through air and the scattering medium as a function of the number of phase conjugations. We find a sharp increase in cosine similarity in the first few iterations, after which we find a constant cosine similarity of 0.4 after only 9 phase conjugations. This behavior corresponds with the simulation results as seen in Fig. 8.2 with a large amount of noise. The cosine similarity is measured on the same sampling grid as the transmission matrix measurements. In (b) and (c) we plot the transmitted field after 8 phase conjugation iterations, propagated through air (b) and the scattering medium (c). The correlations between the fields are hard to identify by eye, due to the low cosine similarity. The difference between the simulated result and the experimental result can be explained by imperfect phase conjugation. The 

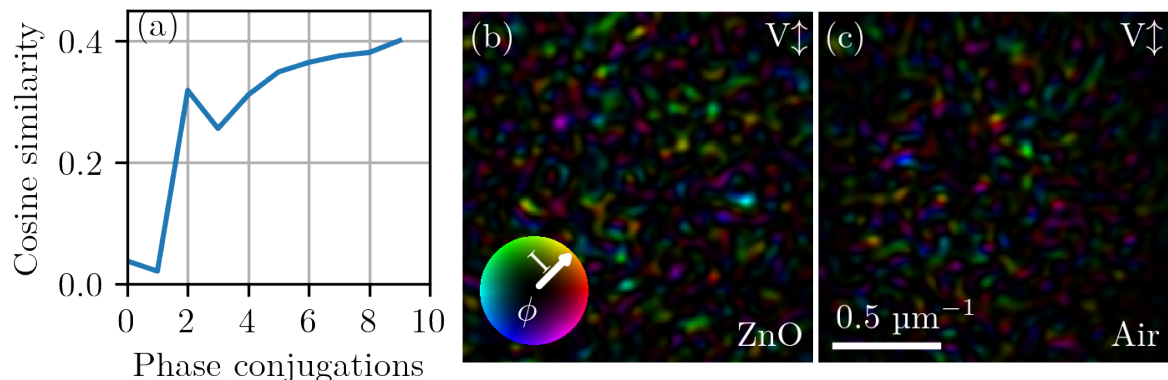

Figure 8.3.: (a) Cosine similarity between field propagated through the air medium and through the scattering medium for iterative phase conjugation as a function of the number of phase conjugations. Two phase conjugations correspond to one iteration of iterative phase conjugation. (b,c) Transmitted field of scattering invariant mode from iterative phase conjugation through $\mathrm{ZnO}(\mathrm{b})$ and through air (c). The field corresponds to iteration 8 of (a). Measurements performed on $2.1 \mu \mathrm{m}$ sample of air and $\mathrm{ZnO}$.

synthesis of the field on S1 and S2 has a limited fidelity, introducing errors in the generated field, any residual misalignment between S1/S2 and D1/D2 also introduces an additional error. Finally, in the phase conjugation experiment there is a power imbalance between the two polarization components, which decreases the fidelity.

Finding scattering invariant modes through iterative phase conjugation has some advantages and disadvantages. From our results, we find lower correlations when finding a scattering invariant mode through optical phase conjugation than through transmission matrix measurements (Chapter 7). The main advantage is that finding a scattering invariant mode through phase conjugation is faster, and requires less measurements. Ten phase conjugations take about 5 minutes, while the measurement of two transmission matrices takes more than an hour. The main disadvantages are that digital optical phase conjugation requires a highly accurate and precise alignment between the field synthesis and field measurement, more sample movement, field synthesis and measurement on both sides of the sample. Finally it only provides a single eigenvector contrary to the full set found in transmission 
matrix measurements. Due to the mutual alignment of a higher number of field synthesis and field measurement planes, phase conjugation measurements more complex to align than a transmission matrix measurement. For our measurements, the sample has a very high time stability and we find higher cosine similarities through transmission matrix measurements. This makes transmission matrix based measurements a better choice for finding scattering invariant mode.

\subsection{Conclusion}

In this chapter, we have demonstrated that we find a scattering invariant mode with an iterative phase conjugation procedure, that alternatively phase conjugates through two distinct mediums. This procedure is similar to a method that we have used to find open transmission channels [1,2]. With a numerical derivation based on measured transmission matrices we demonstrate how an offset field ensures convergence and robustness to noise. By implementing the iterative phase conjugation procedure, we experimentally identify a scattering invariant mode with a cosine similarity of up to 0.4 , after only 9 phase conjugations. While this method is very fast, it is technically demanding and the best achieved cosine similarity is lower than with oud transmission matrix method. 


\section{Bibliography}

[1] X. Hao, L. Martin-Rouault, and M. Cui, "A self-adaptive method for creating high efficiency communication channels through random scattering media," Sci. Rep. 4, 5874 (2014).

[2] J. Bosch, S. A. Goorden, and A. P. Mosk, "Frequency width of open channels in multiple scattering media," Opt. Express 24, 26472-26478 (2016).

[3] A. Andreev, "The thermal conductivity of the intermediate state in superconductors," Zh. Eksp. Teor. Fiz. 46, 1823-1828 (1964).

[4] M. Blaauboer, "On optical phase conjugation and andreev reflection." PhD thesis, Vrije Universiteit Amsterdam (1998).

[5] C. Beenakker, "Why does a metal-superconductor junction have a resistance?" in "Quantum mesoscopic phenomena and mesoscopic devices in microelectronics," (Springer, 2000), pp. 51-60.

[6] S. Börm and C. Mehl, Numerical methods for eigenvalue problems (Walter de Gruyter, 2012).

[7] R. von Mises and H. Pollaczek-Geiringer, "Praktische verfahren der Gleichungsauflösung." Z. Angew. Math. Mech. 9, 152-164 (1929).

[8] S. A. Goorden, "Reversible scattering of light exploited for quantumsecure authentication," $\mathrm{PhD}$ thesis, Universiteit Twente, Enschede (2015).

[9] E. G. van Putten, "Disorder-enhanced imaging with spatially controlled light," PhD thesis, Universiteit Twente, Enschede (2011). 


\section{A. Appendix}

\section{A.1. Speckle correlation function}

The transmitted intensity through a random sample fluctuates as we probe different realizations of the ensemble of random scatterer configurations. The speckle correlation function $C\left(k_{1}, k_{2}, k_{3}, k_{4}, \Delta \omega\right)$ is the intensity-intensity correlation function between incident and outgoing wavevectors $k_{1}, k_{2}, k_{3}, k_{4}$ [1]. The speckle correlation function can be separated into three different contributions: $C^{(1)}, C^{(2)}$, and $C^{(3)}$ [1-3], which correspond to different terms in a diagrammatic expansion, and are also of different order in the dimensionless conductance of the sample $g$, where $g$ is a measure of the number of open eigenchannels the sample supports. The different correlation functions require different experimental geometries to be measured [3]. The $C^{(3)}$ correlation function is measured by injecting light through all wavevectors, and collecting the light for all transmitted wavevectors. It is related to universal conductance fluctuations in electronic nanowires [3,4]. Since it is proportional to $g^{-2}$, which is small in our experimental situation, it is not considered further in this thesis. The $C^{(2)}$ correlation function which is proportional to $1 / g$ describes the fluctuations of the total transmittance of single mode incident light. In the case where only a handful of open eigenchannels dominate the transport (i.e. small $g$ ) this can be a strong contribution. It can be measured in a configuration with with a single incident wavevector and summing over all transmitted wavevectors, which can be performed in optics with a total integrating sphere. Finally the $C^{(1)}$ which is of order 1 , and therefore typically by far the leading term, is the function that describes the short-range intensity fluctuations typical of laser speckle. It can be measured by measuring the correlation between a single incident wavevector and a single transmitted wavevector. 


\section{A.2. Zernike polynomials}

The Zernike polynomials are a basis set of polynomials that are orthogonal on the unit circle [5]. The different Zernike modes correspond to different optical aberrations such as defocus or astigmatism [6]. While they are not the most efficient basis to compensate for aberrations made up of statistically independent modes [7], they are easily computed. This makes them a convenient choice for the correction of imaging aberrations and they are intensively used in the field of adaptive optics [8]. The Zernike polynomials are applied for the correction of imaging defects as a phase correction to the field. This correction can be applied in dynamic or static ways, for example deformable mirrors, spatial light modulators or specifically cut optics that correct for known wavefront distortions.

In Figure A.1 we plot the local phase of the second to tenth Zernike polynomials, according to their Noll index [7]. The Zernike polynomials are labeled according to the corresponding optical aberrations [6]. 


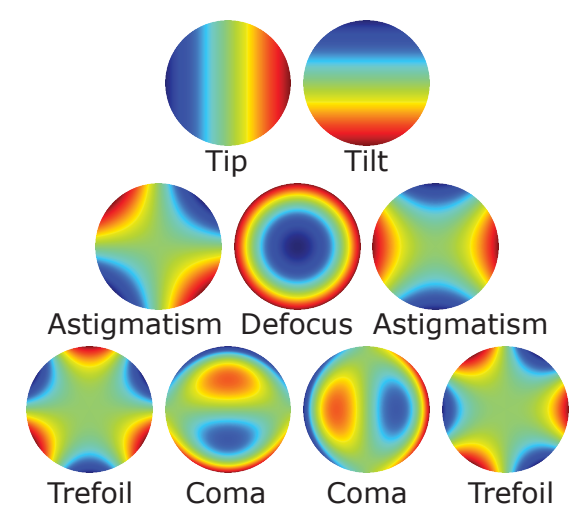

Figure A.1.: Phase of the second to tenth Zernike polynomials according to their Noll index. The Zernike polynomials are labeled according to the typical imaging aberration they correct for. The first Zernike polynomial is not shown as it is constant, corresponding to an overal phase shift. Adaptive optics systems can operate with a limited set of Zernike modes because the amplitude of aberrations typically decreases fast with the order of the Zernike polynomial. In this thesis we use Zernike polynomials up to order 13 to parameterize and correct the aberrations. 


\section{Bibliography}

[1] R. Berkovits and S. Feng, "Correlations in coherent multiple scattering," Physics Reports 238, 135 - 172 (1994).

[2] S. Feng, C. Kane, P. A. Lee, and A. D. Stone, "Correlations and fluctuations of coherent wave transmission through disordered media," Phys. Rev. Lett. 61, 834-837 (1988).

[3] M. C. W. van Rossum and T. M. Nieuwenhuizen, "Multiple scattering of classical waves : microscopy, mesoscopy, and diffusion," Rev. Mod. Phys. 71, 313-371 (1999).

[4] C. W. J. Beenakker, "Random-matrix theory of quantum transport," Rev. Mod. Phys. 69, 731-808 (1997).

[5] F. Zernike, "Beugungstheorie des schneidenver-fahrens und seiner verbesserten form, der phasenkontrastmethode," Physica 1, 689 - 704 (1934).

[6] J. M. Beckers, P. Léna, O. Lai, P.-Y. Madec, G. Rousset, M. Séchaud, M. J. Northcott, F. Roddier, J.-L. Beuzit, F. Rigaut, and D. G. Sandler, Adaptive optics in astronomy (Cambridge University Press, 1999).

[7] R. J. Noll, "Zernike polynomials and atmospheric turbulence*," J. Opt. Soc. Am. 66, 207-211 (1976).

[8] J. W. Hardy, Adaptive optics for astronomical telescopes, vol. 16 (Oxford University Press on Demand, 1998). 


\section{Summary and outlook}

In the work described in this thesis, we have investigated the transport of light through a random scattering medium. We have measured properties of three representations of light transport: transmission channels, time delay eigenstates and scattering invariant modes.

We have described our multispectral transmission matrix measurement procedure, which allows us to sample the transmission operator of a scattering medium. We introduce a figure of merit for the fidelity of the transmission matrix measurement that quantifies how well we sample the transmission properties of the sample. We show the results of our high fidelity multispectral transmission matrix measurements, which allow us to calculate spectral, temporal and transmission properties of transmitted light. This allows us to get an unprecedented insight in the transmission properties of a multiple scattering medium.

For the transmission channels, we have found that the spectral shape of the transmittance can be described by three distinct regimes. The first regime is that of the transmission channels with a transmittance above the average transmittance. These transmission channels can be described by a single spectral shape, that is independent on sample thickness or the transmittance of the transmission channel. We find that the exact shape of the curve depends on the transmission matrix fidelity. The second regime is that of the transmission channels with a transmittance around the sample-average transmittance. For these channels we find the surprising result that the transmittance increases through detuning, even for channels that have a transmittance that is slightly higher than average. Finally we find that the transmission channels with below average transmittance have an increased spectral bandwidth. These nontrivial behaviors are not yet described by current theoretical work, and require theory and simulations.

We have performed the first optical measurement of the time delay of the transmission channels of a multiple scattering medium. We find that the 
most open transmission channels are transmitted slightly faster than the channels with the sample-average transmittance, however the difference is small compared to the average delay time for transmission of light through the sample.

We have performed the first optical measurements of the time delay eigenvalues of a multiple scattering medium. We find that the time delay eigenvalues calculated from the reduced time delay operator range between 0 and 3 times the Thouless time. We also find that the time delay eigenstates have a transmittance that is lower than the average sample transmittance, and typically decorrelate faster than the speckle correlation function.

As a final subject we have studied scattering invariant modes, which are fields that produce a similar transmitted field whether they are propagated through a scattering medium or through a layer of air. We show an effective method of calculating the scattering invariant modes from the transmission matrices of two materials. We have demonstrated sparse scattering invariant modes, that can transmit the same simple image through two distinct materials. Finally we have demonstrated a procedure for finding a scattering invariant mode with an iterative phase conjugation procedure, which quickly finds a scattering invariant mode without requiring any transmission matrix measurements.

As an outlook, our results show that the spectral bandwidth of the transmission of transmission channels can be very broad for some channels at high fidelity and reverts to the Thouless width for low fidelity measurements. This means that a transmission channels always has a bandwidth that is at least equal to the Thouless width. This is important for the application of transmission channels where light is injected deep into or through a medium such as light activated medicine, secure authentication [1], pulse transmission, and imaging inside or through a scattering material [2-5]. The time delay eigenstates can be used in practical applications that are depend on the interaction between light and matter, such as energy conversion, spectroscopy and optical sensors. Our experimental method can be expanded from measuring transmission matrices to measurements of a multispectral $S$-matrix, which encompasses the transmission matrices and reflection matrices in both directions. By accurately mapping the basis of the transmission matrix to that of the reflection matrix, as is done in the phase conjugation, it is possible to directly link transmission channels to reflection channels. The measurement 
of a the $S$-matrix will enable elucidation of the full time delay eigenstates and of correlations between the reflection and the transmission channels [6], which allows for verification of new theoretical predictions as well as potential new approaches to imaging, spectroscopy and metrology in scattering systems. 


\section{Bibliography}

[1] S. A. Goorden, M. Horstmann, A. P. Mosk, B. Skoric, and P. Pinkse, "Quantum-secure authentication of a physical unclonable key," Optica 1, 421-424 (2014).

[2] I. Freund, "Looking through walls and around corners," Physica A 168, 49-65 (1990).

[3] S. Popoff, G. Lerosey, M. Fink, A. C. Boccara, and S. Gigan, "Image transmission through an opaque material," Nat. Commun. 1, 81 (2010).

[4] A. P. Mosk, A. Lagendijk, G. Lerosey, and M. Fink, "Controlling waves in space and time for imaging and focusing in complex media," Nat. Photonics 6, 283 (2012).

[5] J. Bertolotti, E. G. van Putten, C. Blum, A. Lagendijk, W. L. Vos, and A. P. Mosk, "Non-invasive imaging through opaque scattering layers," Nature 491, 232-234 (2012).

[6] A. M. Paniagua-Diaz, I. Starshynov, N. Fayard, A. Goetschy, R. Pierrat, R. Carminati, and J. Bertolotti, "Blind ghost imaging," Optica 6, 460464 (2019). 


\section{Samenvatting}

In dit proefschrift onderzoeken we het transport van licht door een meervoudig verstrooiend materiaal. We rapporteren metingen van de eigenschappen van drie representatieve toestanden van licht transport: transmissie kanalen, tijdsvertraging eigentoestaanden en verstrooingsonafhankelijke toestanden (scattering invariant modes of SIMs).

We beschrijven een procedure voor het meten van de multispectrale transmissiematrix (TM), waarmee we de transmissie operator van een veelvoudig verstrooiend medium bemonsteren. We introduceren een maat voor de precisie $F$ van de transmissiematrix meting, die kwantificeert hoe goed we de transmissie eigenschappen van een monster bemonsteren. We laten resultaten zien met onze hoge precisie $F$, waarmee we de spectrale-, tijdsafhankelijke-, en transmissie-eigenschappen van een monster kunnen berekenen. Dit geeft ons een ongekend inzicht in de transmissie-eigenschappen van een veelvoudig verstrooiend medium.

Voor de transmissie kanalen vinden we drie verschillende gedragingen voor de spectraallijn van de transmissie. Eerst kijken we naar transmissie kanalen met een transmissie hoger dan het gemiddelde. Voor deze kanalen vinden we een enkele curve die de spectraallijn beschrijft, onafhankelijk van de monsterdikte of de transmissie van het transmissie kanaal. Vervolgens kijken we naar de transmissie kanalen met een transmissie rond het gemiddelde. Voor deze kanalen vinden we het verrassende resultaat dat de transmissie toeneemt na verstemmen, zelfs voor kanalen met een transmissie boven het monster-gemiddelde. Ten slotte vinden we dat de kanalen met een transmissie lager dan de gemiddelde transmissie van het monster een toename hebben in de lijnbreedte van de transmissie. Deze niet triviale gedragingen worden op moment nog niet beschreven door de huidige theorieën, waarvoor nieuwe theorieën en simulaties nodig zijn.

We hebben de eerste optische metingen van de tijdsvertraging van de transmissie kanalen van een veelvoudig verstrooiend medium uitgevoerd. Hierin 
vinden wij dat de kanalen met de hoogste transmissie een kleinere tijdsvertraging ondervinden. Het verschil is echter klein vergeleken met de gemiddelde tijdsvertraging van lichttransmissie door het monster.

We presenteren de eerste optische metingen van de tijdvertraging eigentoestanden van een veelvoudig verstrooiend medium. We vinden tijdvertraging eigenwaardes, die we berekenen op basis van de gereduceerde tijdvertragingsoperator, tussen 0 en 3 keer de Thouless tijd. Ook zien we dat de tijdvertraging eigentoestanden een lager dan monster-gemiddelde transmissie hebben, en sneller decorreleren dan de specklecorrelatiefunctie.

Ten slotte bestuderen we de verstrooiingsonafhankelijke toestanden (scattering invariant modes of SIMs), dit zijn lichtvelden die hetzelfde uit een verstrooiend medium en een laag lucht komen. We demonstreren een effectieve methode om uit de transmissiematrices van twee materialen de verstrooiingsonafhankelijke toestanden te berekenen. Ook demonstreren we de zogenoemde sparse SIMs, waarmee SIMs construeren die een simpele afbeeldingen door twee materialen projecteert. Ten slotte demonstreren we een methode om door iteratieve faseconjugatie snel een verstrooiingsonafhankelijke toestand te vinden.

Onze resultaten laten zien dat de spectrale lijnbreedte van de transmissie van transmissiekanalen erg hoog kan zijn voor kanalen gemeten met een hoge precisie $F$ en dat deze gelijk is aan de Thouless breedte voor metingen met een lage precisie $F$. Dit betekend dat de lijnbreedte van de toename van de transmissie altijd ten minste even groot is als de Thouless breedte. Dit is belangrijk voor toepassingen die gebruik maken van transmissie kanalen, zoals medicijnen die geactiveerd worden door licht, kwantumveilige authenticatie [1], pulstransmissie en afbeeldingstechnieken in of door verstrooiende materialen [2-5]. De tijdvertragings eigentoestandne kunnen gebruikt worden in praktische toepassingen die gebruik maken van de interactie tussen licht en materie, zoals energie omzettingen, spectroscopie en optische sensoren.

De experimentele technieken kunnen uitgebreid worden van het meten van transmissie matrices tot het meten van een multispectrale $S$-matrix, die zowel de transmissiematrices in beide richtingen als de reflectiematrices bevat. Door het accuraat afbeelden van de basis van de transmissiematrix op de basis van de reflectiematrices, zoals wij hebben gedaan voor de faseconjugatie metingen, is het mogelijk om correlaties tussen transmissiekanalen en 
reflectiekanalen te vinden [6]. Met een meting van de $S$-matrix is het mogelijk om de volledige tijdvertragings eigentoestanden te meten ook maakt het de verificatie van nieuwe theoretische voorspellingen, en nieuwe afbeeldingstechnieken en spectroscopische metingen mogelijk. 


\section{Bibliografie}

[1] S. A. Goorden, M. Horstmann, A. P. Mosk, B. Skoric, and P. Pinkse, "Quantum-secure authentication of a physical unclonable key," Optica 1, 421-424 (2014).

[2] I. Freund, "Looking through walls and around corners," Physica A 168, 49-65 (1990).

[3] S. Popoff, G. Lerosey, M. Fink, A. C. Boccara, and S. Gigan, "Image transmission through an opaque material," Nat. Commun. 1, 81 (2010).

[4] A. P. Mosk, A. Lagendijk, G. Lerosey, and M. Fink, "Controlling waves in space and time for imaging and focusing in complex media," Nat. Photonics 6, 283 (2012).

[5] J. Bertolotti, E. G. van Putten, C. Blum, A. Lagendijk, W. L. Vos, and A. P. Mosk, "Non-invasive imaging through opaque scattering layers," Nature 491, 232-234 (2012).

[6] A. M. Paniagua-Diaz, I. Starshynov, N. Fayard, A. Goetschy, R. Pierrat, R. Carminati, and J. Bertolotti, "Blind ghost imaging," Optica 6, 460464 (2019). 


\section{Dankwoord}

Promoveren is meer dan alleen van het schrijven van een proefschrift, en geen enkele wetenschapper zit alleen in een ivoren toren. Het samenwerken met collega's en studenten is essentieel, zowel om persoonlijk te ontwikkelen als voor het onderzoek. Er zijn dan ook vele collega's en vrienden die ik hiervoor dankbaar ben.

Als eerste wil ik graag mijn promotor Allard bedanken. Allard, je hebt een fijne stijl van werken, die niet werkt op micromanagement, maar veel initiatief laat. Dit heb ik erg kunnen waarderen, hoewel het soms ook uitdagender is. Je enthousiasme en deskundigheid in de natuurkunde is bewonderenswaardig en de soms ietwat lange overleggen waren altijd erg leerzaam.

Ook Pritam - mijn projectpartner heeft een belangrijke rol gespeeld tijdens dit proefschrift. Pritam, de samenwerking met jou is heel waardevol. Het gemak om bij jou het kantoor binnen te lopen en te brainstormen over ideeën was een vanzelfsprekendheid en ontzettend waardevol - en de meeste hoofdstukken in dit proefschrift leunen ook op de door jou ontwikkelde transmissie matrix code. Sociaal ben je belangrijk in de groep. Je enthousiasme voor sport, ook samen met Sebas, en schijnbaar onuitputtelijke lijst van sporten heeft ook mij geïnspireerd om actiever en sportiever te zijn, en geleid tot vele sportieve groepsactiviteiten.

Ik wil ook mijn co-promotor Sanli bedanken. Sanli, je hebt veel gedaan om de groep te organiseren toen we opstartten in Utrecht. We begonnen met theepauzes, die later helaas minder werden, maar opnieuw opgestart zijn tijdens de thuiswerkperiode om toch sociaal contact actief te houden binnen de groep. Ook in de organisatie en het opgeruimd houden van de groep en de groepsoverleggen. Ook het fab lab, en de daarbij horende lasersnijder en 3d-printer waren erg handig.

Natuurlijk waren er ook mijn kantoorgenoten. Marcel, met een onuitputtelijke blik op de buitenlandse en binnenlandse politiek, gezond optimisme en ongelofelijk brede pub quiz-kennis. Jasper, je hebt een bijzondere pas- 
sie voor fietsen, die mij ook een klein beetje geïnfecteerd heeft. Het stukje fietsen was goed om tot rust te komen na een drukke dag.

Binnen een experimentele vakgroep is de ondersteuning door vakmensen van levensbelang. Ik bedank dan ook Cees voor het optische- en algemene advies, en hoop dat je kan gaan genieten van je pensioen. De ondersteuning van Dante houdt onze experimenten veilig, en de metingen ruisloos. Bij Paul kan je altijd terecht voor een kort praatje, de organisatie van onze gezellige groepsborrels en op maat gemaakte stukken uit de werkplaats. Ook de secretariële ondersteuning van Mijke kunnen we niet vergeten, Mijke je hebt een belangrijke sociale plaats in de groep, en zorgt dat alles blijft functioneren, bedankt hiervoor.

Ook alle andere collega's hebben hun plaats gehad tijdens deze promotie. Ik wil Sebas bedanken, voor zijn leidende rol in de sociale cohesie in de groep. Dashka, we had a fun trip to the Ikea, don't forget to buy yourself some small luxuries every now and then. Jacob, je openheid en onbevooroordeelde blik op de wereld is bewonderenswaardig. Dries, je combinatie van gezonde werktijden, een academische carrière en gedeeld enthousiasme voor proefjes zoals een plasma in de magnetron zijn fantastisch. Ik wil ook mijn collega's Arjon, Bohdan, Dorian, Frits, Pegah, Peter, Karindra, en Zhu, de bacheloren master studenten die ik begeleid heb, Suzan en Tommaso, en voormalige collega's Sergei, Jin, Floor, Sid, Abhilash, Said, Javi, Arfor, Xiaoqing, Ole en Sandy bedanken voor hun bijdrages aan de groep en dit werk.

Our collaborations brought great insights and progress to our work. For this, I would like to thank Stefan and Matthias from Vienna and Alexey, Wade, Hasan and Hui in the USA. It was also a joy to work once again with Hasan, after we both moved away from Twente to a different university.

Ook mijn vrienden buiten de universiteit zijn erg belangrijk geweest en wil ik bedanken. Chris, de 'Brabanders': Diana and Sasha, Jin and Yue, and Devashish. En mijn optica-buddies Reinout, Bob en Lieke.

Ten slotte wil ik mijn familie bedanken voor hun steun en liefde. 


\section{Over de auteur}

Jeroen Bosch is geboren op 14 september 1992 in Davos, Zwitserland. Van 2004 tot 2010 doorliep hij het Voortgezet Wetenschappelijk Onderwijs aan het Pius X College, te Almelo. In de laatste twee schooljaren was hij lid van de medezeggenschapsraad. Hierna heeft hij tussen 2010 en 2015 de bachelor Technische Natuurkunde en de master Applied Physics aan de Universiteit Twente cum laude afgerond. Tijdens de laatste vier studiejaren was hij lid van de faculteitsraad technische natuurwetenschappen. Zijn masterscriptie "Spectroscopic measurements on the width of open transmission channels" ging over de propagatie van licht in meervoudig verstrooiende materialen, onder begeleiding van Allard Mosk. In dit afstudeerwerk heeft hij een digitale fase conjugatie opstelling in gebruik genomen, en hiermee open transmissie kanalen van een verstrooiend materiaal gevonden door iteratieve faseconjugatie. Voor zijn masterscriptie kreeg Jeroen de Shell Afstudeerprijs voor Natuurkunde door de Koninklijke Hollandse Maatschappij der Wetenschappen uitgereikt.

Dit proefschrift is het resultaat van het promotieonderzoek aan de Universiteit Utrecht. Tijdens zijn aanstelling heeft hij een bachelor en een masterstudent begeleid bij hun scriptie, en geassisteerd bij het University College Utrecht Golven en optica practicum en het practicum Statistische fysica. 
